\title{
Ruthenium-Catalyzed Propargylic Reduction of Propargylic Alcohols with Hantzsch Ester
}

Haowei Ding, ${ }^{a}$ Ken Sakata, ${ }^{\text {b }}$ Shogo Kuriyama, ${ }^{a}$ and Yoshiaki Nishibayashi*a

ken.sakata@phar.toho-u.ac.jp, ynishiba@sys.t.u-tokyo.ac.jp

${ }^{a}$ Department of Systems Innovation, and School of Engineering, The University of Tokyo, Hongo, Bunkyo-ku, Tokyo 113-8656, Japan.

${ }^{b}$ Faculty of Pharmaceutical Sciences, Toho University, Miyama, Funabashi, Chiba 274-8510, Japan. 


\section{Table of Contents}

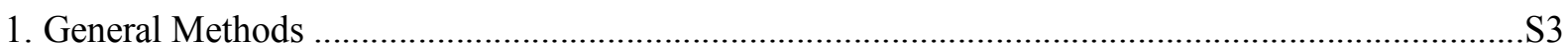

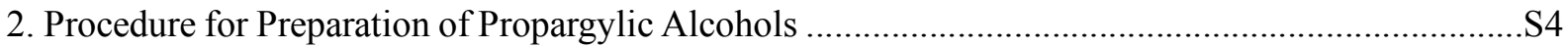

3. Ruthenium-Catalyzed Propargylic Reduction of Propargylic Alcohols (2) with Hantzsch

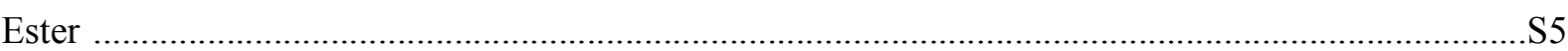

4. Large-Scale Reaction of Ruthenium-Catalyzed Propargylic Reduction of Propargylic Alcohols (2a)

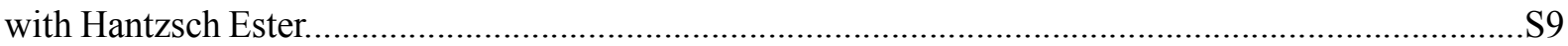

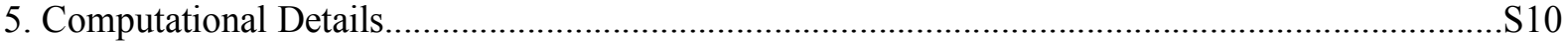

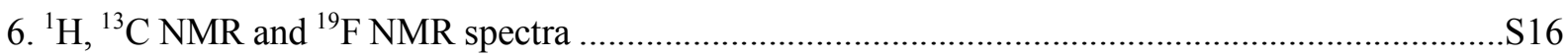

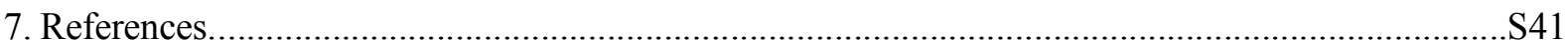




\section{General Methods}

${ }^{1} \mathrm{H}$ NMR (400 MHz), ${ }^{13} \mathrm{C}$ NMR (100 MHz), and ${ }^{19} \mathrm{~F}$ NMR (376 MHz) spectra were recorded on a JEOL ECS-400 spectrometer in suitable solvents. The data are reported as $(\mathrm{s}=$ singlet, $\mathrm{d}=$ doublet, $\mathrm{dd}$ $=$ double doublet, $\mathrm{dt}=$ double triplet, $\mathrm{t}=$ triplet, $\mathrm{q}=$ quartet, $\mathrm{br}=$ broad, $\mathrm{m}=$ multiplet or unresolved, coupling constant(s) in Hz, integration). Mass spectra were measured on a JEOL JMS-700 mass spectrometer. Commercially obtained reagents were used without further purification. All reactions were carried out under dry nitrogen atmosphere by using standard Schlenk techniques. Solvents were dried by general methods and degassed before use. Propargylic alcohols 1-phenyl-2-propyn-1-ol (2b), 1,1-bis(4-methoxyphenyl)-2-propyn-1-ol $\quad(\mathbf{2 t}), \quad$ 1,3-diphenyl-2-propyn-1-ol $\quad \mathbf{( 2 x}), \quad \mathrm{NH}_{4} \mathrm{BF}_{4}, \quad$ and ethynylmagnesium bromide are commercially available. Ruthenium catalysts $\mathbf{1 a}$ and $\mathbf{1 b}$ were prepared according to the literature procedures. ${ }^{\mathrm{S} 1}$ Preparation of Hantzsch esters $\mathbf{3}^{\mathrm{S} 2}$ and $\mathbf{3}-\mathrm{d}_{2}{ }^{\mathrm{S} 3}$ and propargylic alcohols 2a $,{ }^{\mathrm{S} 4} \mathbf{2 c},{ }^{\mathrm{S} 5} \mathbf{2 e},{ }^{\mathrm{S} 5} \mathbf{2 f},{ }^{\mathrm{S} 4} \mathbf{2 g},{ }^{\mathrm{S} 4} \mathbf{2 h},{ }^{\mathrm{S} 6} \mathbf{2 i},{ }^{\mathrm{S} 4} \mathbf{2} \mathbf{k},{ }^{\mathrm{S} 7} \mathbf{2 l},{ }^{\mathrm{S} 8} \mathbf{2} \mathbf{m},{ }^{\mathrm{S} 9} \mathbf{2 n},{ }^{\mathrm{S} 10} \mathbf{2 0},{ }^{\mathrm{S} 11} \mathbf{2} \mathbf{p},{ }^{\mathrm{S} 5} \mathbf{2 q},{ }^{\mathrm{S} 12} \mathbf{2 r},{ }^{\mathrm{S} 13} \mathbf{2} \mathbf{s},{ }^{\mathrm{S} 14} \mathbf{2 v},{ }^{\mathrm{S} 15}$ and $\mathbf{2} \mathbf{w}^{\mathrm{S} 16}$ were reported elsewhere, by-product 1,3 -enynes $\mathbf{6} \mathbf{v}^{\mathrm{S} 17}$ and $\mathbf{6} \mathbf{w}^{\mathrm{S} 18}$ were reported elsewhere. Other reagents were purchased commercially and used as received. 


\section{Procedure for Preparation of Propargylic Alcohols}

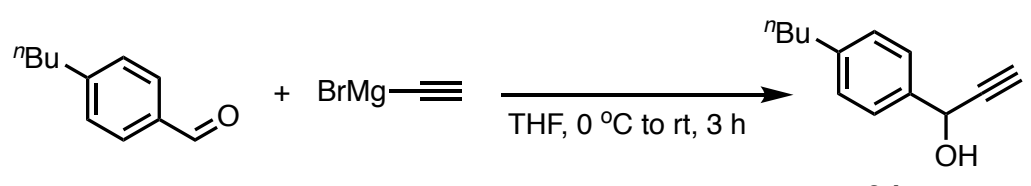

2d

A typical experiment procedure of the preparation of a propargylic alcohol, 1-(4-butylphenyl)2-propyn-1-ol (2d) is described below. In a $50 \mathrm{~mL}$ Schlenk flask were placed 4-butylbenzaldehyde (0.49 g, $3.0 \mathrm{mmol})$ and anhydrous THF $(12 \mathrm{~mL})$ under $\mathrm{N}_{2}$ atmosphere and the solution was cooled down to 0 ${ }^{\circ} \mathrm{C}$. To the solution, ethynylmagnesium bromide $(0.5 \mathrm{M}$ in THF, $6.6 \mathrm{~mL}, 3.3 \mathrm{mmol})$ was added dropwise, and the mixture was stirred for $10 \mathrm{~min}$. The mixture was warmed up to room temperature and was stirred for 3 hours. After the reaction, the mixture was quenched with saturated $\mathrm{NH}_{4} \mathrm{Cl}$ aq., and the solution was extracted with EtOAc (5 mL x 3). The combined organic layers were dried over anhydrous $\mathrm{MgSO}_{4}$ and concentrated under reduced pressure. The residue was purified by column chromatography $\left(\mathrm{SiO}_{2}\right)$ with hexane/EtOAc (9:1) as eluent to give $\mathbf{2 d}$ as a pale yellow oil $(0.485 \mathrm{~g}, 2.57 \mathrm{mmol}, 86 \%$ isolated yield). ${ }^{1} \mathrm{H}$ NMR $\left(\mathrm{CDCl}_{3}\right): \delta$ 7.47-7.45 (m, 2H), 7.21-7.19 (m, 2H), $5.44(\mathrm{dd}, J=6.3$ and $2.0 \mathrm{~Hz}, 1 \mathrm{H})$, $2.66(\mathrm{~d}, J=2.0 \mathrm{~Hz}, 1 \mathrm{H}), 2.62(\mathrm{t}, J=7.8 \mathrm{~Hz}, 2 \mathrm{H}), 2.17$ (d, $J=6.4 \mathrm{~Hz}, 1 \mathrm{H}), 1.63-1.55$ (m, 2H), $1.40-1.31$ (m, 2H), $0.92(\mathrm{t}, J=7.2 \mathrm{~Hz}, 3 \mathrm{H}) .{ }^{13} \mathrm{C} \mathrm{NMR}\left(\mathrm{CDCl}_{3}\right): \delta 143.7,137.4,128.9,126.7,83.7,74.8,64.5$, 35.5, 33.7, 22.5, 14.1. HRMS (FAB+): Calcd. for $\mathrm{C}_{13} \mathrm{H}_{16} \mathrm{O}[\mathrm{M}]$ 188.1201. Found 188.1192.

\section{Spectroscopic Data and Isolated Yields (NMR yields) of Other Products}

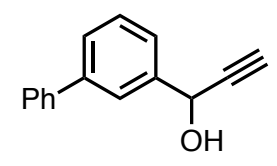

1-(1,1'-Biphenyl-3-yl)-2-propyn-1-ol (2j): $0.506 \mathrm{~g}, 81 \%$ yield, a pale yellow oil. ${ }^{1} \mathrm{H} \mathrm{NMR}\left(\mathrm{CDCl}_{3}\right): \delta$ 7.790-7.786 (m, 1H), 7.62-7.54 (m, 4H), 7.49-7.43 (m, 3H), 7.39-7.34 (m, 1H), $5.54(\mathrm{dd}, J=6.0$ and $2.4 \mathrm{~Hz}, 1 \mathrm{H}), 2.69(\mathrm{~d}, J=2.4 \mathrm{~Hz}, 1 \mathrm{H}), 2.31(\mathrm{~d}, J=6.0 \mathrm{~Hz}, 1 \mathrm{H}) .{ }^{13} \mathrm{C} \mathrm{NMR}\left(\mathrm{CDCl}_{3}\right): \delta 141.9,140.9,140.6$, 129.3, 129.0, 127.6, 127.5, 127.4, 125.6, 83.5, 75.2, 64.6. HRMS (FAB+): Calcd. for $\mathrm{C}_{15} \mathrm{H}_{12} \mathrm{O}[\mathrm{M}]$ 208.0888. Found 208.0880.

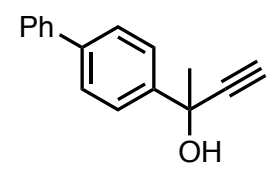

2-(1,1'-Biphenyl-4-yl)-3-butyn-2-ol (2u): $0.526 \mathrm{~g}, 79 \%$ yield, a whit solid, m.p: 83.5-85.2 ${ }^{\circ} \mathrm{C} .{ }^{1} \mathrm{H}$ NMR $\left(\mathrm{CDCl}_{3}\right): \delta$ 7.77-7.50 (m, 2H), 7.63-7.61 (m, 4H), 7.49- $7.37(\mathrm{~m}, 3 \mathrm{H}), 2.73(\mathrm{~s}, 1 \mathrm{H}), 2.70(\mathrm{~s}, 1 \mathrm{H}), 1.86(\mathrm{~s}$, $3 \mathrm{H}) .{ }^{13} \mathrm{C} \mathrm{NMR}\left(\mathrm{CDCl}_{3}\right): \delta 144.1,140.9,140.7,128.9,127.6,127.2,125.5,87.3,73.3,69.8,33.2$. HRMS $(\mathrm{FAB}+)$ : Calcd. for $\mathrm{C}_{16} \mathrm{H}_{14} \mathrm{O}[\mathrm{M}] 222.1045$. Found 222.1043. 
3. Ruthenium-Catalyzed Propargylic Reduction of Propargylic Alcohols (2) with Hantzsch Ester<smiles>C#CC(O)c1ccc(-c2ccccc2)cc1</smiles>

2a<smiles>CC1=C(C)C(F)C(C)=C(C)N1</smiles>

3 (1.0 equiv) $\left(\mathrm{E}=\mathrm{CO}_{2} \mathrm{Et}\right)$

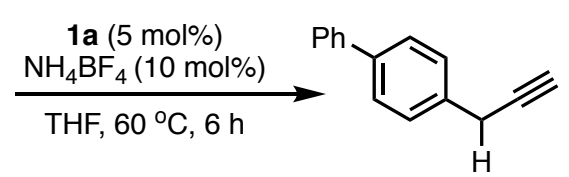

4a

A typical experiment procedure for the reaction of 1-(1,1'-biphenyl-4-yl)-2-propyn-1-ol (2a) with Hantzsch ester 3 is described below. In a $20 \mathrm{~mL}$ Schlenk flask were placed ruthenium catalyst 1a (8.0 $\mathrm{mg}, 0.0125 \mathrm{mmol}$ ) and $\mathrm{NH}_{4} \mathrm{BF}_{4}(2.6 \mathrm{mg}, 0.0250 \mathrm{mmol})$ under $\mathrm{N}_{2}$. Anhydrous THF (2.5 mL) was added, and then $2 \mathrm{a}(52.0 \mathrm{mg}, 0.250 \mathrm{mmol})$ and $\mathbf{3}(63.3 \mathrm{mg}, 0.250 \mathrm{mmol})$ were added. The mixture was stirred at $60{ }^{\circ} \mathrm{C}$ for 6 hours. The solvent was removed under reduced pressure, and the residue was purified by the column chromatography $\left(\mathrm{SiO}_{2}\right)$ with hexane/EtOAc (99:1) as eluent to give 4-(2-propynyl)-1,1'biphenyl (4a) ${ }^{\mathrm{S} 19}$ as a pale yellow solid (47.4 $\mathrm{mg}, 0.247 \mathrm{mmol}, 99 \%$ isolated yield). ${ }^{1} \mathrm{H} \mathrm{NMR}\left(\mathrm{CDCl}_{3}\right): \delta$ 7.63-7.59 (m, 4H), 7.49-7.45 (m, 4H), 7.39-7.36 (m, 1H), 3.69 (d, $J=2.5 \mathrm{~Hz}, 2 \mathrm{H}), 2.25(\mathrm{t}, J=2.5 \mathrm{~Hz}$, $1 \mathrm{H}) .{ }^{13} \mathrm{C} \mathrm{NMR}\left(\mathrm{CDCl}_{3}\right): \delta 140.9,139.9,135.3,128.9,128.4,127.5,127.4,127.2,82.0,70.7,24.6$.

\section{Spectroscopic Data and Isolated Yields (NMR yields) of Other Products}

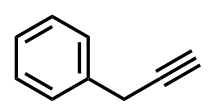

3-Phenyl-1-propyne (4b) ${ }^{\mathrm{S} 19}: 16.6 \mathrm{mg}, 57 \%$ isolated yield (99\% NMR yield). A yellow oil. ${ }^{1} \mathrm{H}$ NMR $\left(\mathrm{CDCl}_{3}\right): \delta 7.36-7.29(\mathrm{~m}, 4 \mathrm{H}), 7.25-7.21(\mathrm{~m}, 1 \mathrm{H}), 3.60(\mathrm{~d}, J=2.6 \mathrm{~Hz}, 2 \mathrm{H}), 2.18(\mathrm{t}, J=2.6 \mathrm{~Hz}, 1 \mathrm{H}) .{ }^{13} \mathrm{C}$ NMR $\left(\mathrm{CDCl}_{3}\right): \delta 136.2,128.7,128.0,126.8,82.1,70.6,24.9$.

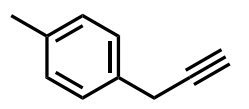

3-(4-Tolyl)-1-propyne (4c) ${ }^{\mathrm{S} 19}: 25.7 \mathrm{mg}, 79 \%$ isolated yield (99\% NMR yield). A pale yellow oil. ${ }^{1} \mathrm{H}$ NMR (CDCl $): \delta 7.25-7.23(\mathrm{~m}, 2 \mathrm{H}), 7.14-7.12(\mathrm{~m}, 2 \mathrm{H}), 3.57(\mathrm{~d}, J=2.6 \mathrm{~Hz}, 2 \mathrm{H}), 2.33(\mathrm{~s}, 3 \mathrm{H}), 2.17(\mathrm{t}$, $J=2.6 \mathrm{~Hz}, 1 \mathrm{H}) .{ }^{13} \mathrm{C} \mathrm{NMR}\left(\mathrm{CDCl}_{3}\right): \delta 136.4,133.2,129.4,127.9,82.4,70.4,24.5,21.2$.

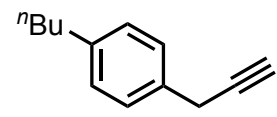

3-(4-Butylphenyl)-1-propyne (4d): $40.7 \mathrm{mg}$, 94\% isolated yield (99\% NMR yield). A pale yellow oil. ${ }^{1} \mathrm{H}$ NMR $\left(\mathrm{CDCl}_{3}\right): \delta$ 7.27-7.13 (m, 4H), $3.58(\mathrm{~d}, J=1.6 \mathrm{~Hz}, 2 \mathrm{H}), 2.59(\mathrm{t}, J=7.6 \mathrm{~Hz}, 2 \mathrm{H}), 2.18-2.17$ (m, $1 \mathrm{H}), 1.62-1.54(\mathrm{~m}, 2 \mathrm{H}), 1.39-1.30(\mathrm{~m}, 2 \mathrm{H}), 0.92(\mathrm{t}, J=7.4 \mathrm{~Hz}, 3 \mathrm{H}) .{ }^{13} \mathrm{C} \mathrm{NMR}\left(\mathrm{CDCl}_{3}\right): \delta 141.6,133.3$, 128.8, 127.8, 82.4, 70.3, 35.6, 33.8, 24.5, 22.5, 14.1. HRMS (FAB+): Calcd. for $\mathrm{C}_{13} \mathrm{H}_{17}[\mathrm{M}+\mathrm{H}]^{+}$ 173.1330. Found 173.1339.

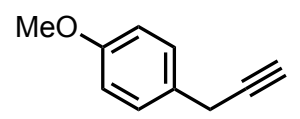

3-(4-Methoxyphenyl)-1-propyne (4e) ${ }^{\mathrm{S} 20}: 29.2 \mathrm{mg}, 80 \%$ isolated yield (99\% NMR yield). A pale yellow oil. ${ }^{1} \mathrm{H}$ NMR $\left(\mathrm{CDCl}_{3}\right): \delta$ 7.27-7.25 (m, 2H), 6.87-6.85 (m, 2H), $3.79(\mathrm{~s}, 3 \mathrm{H}), 3.54(\mathrm{~d}, J=2.6 \mathrm{~Hz}, 2 \mathrm{H})$, $2.16(\mathrm{t}, J=2.6 \mathrm{~Hz}, 1 \mathrm{H}) .{ }^{13} \mathrm{C} \mathrm{NMR}\left(\mathrm{CDCl}_{3}\right): \delta 158.5,129.0,128.3,114.1,82.6,70.3,55.4,24.1$. 


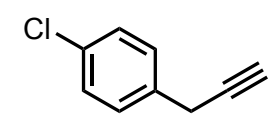

1-Chloro-4-(2-propyn-1-yl)benzene (4f) ${ }^{\mathrm{S} 19}: 27.1 \mathrm{mg}, 72 \%$ isolated yield (99\% NMR yield). A pale yellow oil. ${ }^{1} \mathrm{H}$ NMR $\left(\mathrm{CDCl}_{3}\right)$ : $\delta 7.29$ (s, $\left.4 \mathrm{H}\right), 3.57(\mathrm{~d}, J=2.8 \mathrm{~Hz}, 2 \mathrm{H}), 2.20(\mathrm{t}, J=2.8 \mathrm{~Hz}, 1 \mathrm{H}) .{ }^{13} \mathrm{C} \mathrm{NMR}$ $\left(\mathrm{CDCl}_{3}\right): \delta 134.7,132.7,129.3,128.8,81.5,71.0,24.4$.

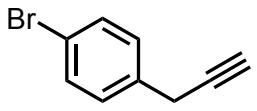

1-Bromo-4-(2-propyn-1-yl)benzene (4g) ${ }^{\mathrm{S} 21}$ : $41.0 \mathrm{mg}$, 84\% isolated yield. A pale yellow oil. ${ }^{1} \mathrm{H}$ NMR $\left(\mathrm{CDCl}_{3}\right): \delta 7.46-7.44(\mathrm{~m}, 2 \mathrm{H}), 7.24-7.22(\mathrm{~m}, 2 \mathrm{H}), 3.56(\mathrm{~d}, J=2.6 \mathrm{~Hz}, 2 \mathrm{H}), 2.20(\mathrm{t}, J=2.6 \mathrm{~Hz}, 1 \mathrm{H}) .{ }^{13} \mathrm{C}$ NMR $\left(\mathrm{CDCl}_{3}\right): \delta 135.2,131.7,129.7,120.7,81.4,71.1,24.2$.

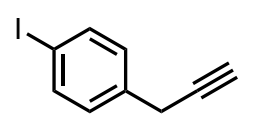

1-Iodo-4-(2-propyn-1-yl)benzene (4h) ${ }^{\mathrm{S} 22}$ : $59.6 \mathrm{mg}$, 99\% isolated yield. A pale yellow solid ${ }^{1} \mathrm{H}$ NMR $\left(\mathrm{CDCl}_{3}\right): \delta 7.65-7.62(\mathrm{~m}, 2 \mathrm{H}), 7.11-7.09(\mathrm{~m}, 2 \mathrm{H}), 3.54(\mathrm{~d}, J=2.6 \mathrm{~Hz}, 2 \mathrm{H}), 2.20(\mathrm{t}, J=2.6 \mathrm{~Hz}, 1 \mathrm{H}) .{ }^{13} \mathrm{C}$ $\operatorname{NMR}\left(\mathrm{CDCl}_{3}\right): \delta 137.7,135.9,130.0,92.1,81.3,71.1,24.5$.

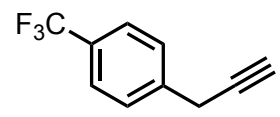

1-(2-Propyn-1-yl)-4-(trifluoromethyl)benzene (4i) ${ }^{\mathrm{S} 23}: 19.8 \mathrm{mg}, 43 \%$ isolated yield (74\% NMR yield). A pale yellow oil. ${ }^{1} \mathrm{H}$ NMR $\left(\mathrm{CDCl}_{3}\right): \delta$ 7.60-7.58 (m, $\left.2 \mathrm{H}\right), 7.49-7.47(\mathrm{~m}, 2 \mathrm{H}), 3.67(\mathrm{~d}, J=2.6 \mathrm{~Hz}, 2 \mathrm{H})$, $2.24(\mathrm{t}, J=2.6 \mathrm{~Hz}, 1 \mathrm{H}) .{ }^{13} \mathrm{C} \mathrm{NMR}\left(\mathrm{CDCl}_{3}\right): \delta 140.3,129.3\left(\mathrm{q},{ }^{2} J_{\mathrm{C}-\mathrm{F}}=32.1 \mathrm{~Hz}\right), 128.4,125.6\left(\mathrm{q},{ }^{3} J_{\mathrm{C}-\mathrm{F}}=\right.$ $3.5 \mathrm{~Hz}), 124.3\left(\mathrm{q},{ }^{1} J_{\mathrm{C}-\mathrm{F}}=270 \mathrm{~Hz}\right), 80.9,71.4,24.9 .{ }^{19} \mathrm{~F} \mathrm{NMR}\left(\mathrm{CDCl}_{3}\right): \delta-62.35$.

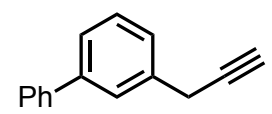

3-(2-Propyn-1-yl)-1,1'-biphenyl (4j): $47.5 \mathrm{mg}, 99 \%$ isolated yield. A pale yellow oil. ${ }^{1} \mathrm{H}$ NMR $\left(\mathrm{CDCl}_{3}\right): \delta 7.61-7.33(\mathrm{~m}, 9 \mathrm{H}), 3.68(\mathrm{~d}, J=2.0 \mathrm{~Hz}, 2 \mathrm{H}), 2.23-2.21(\mathrm{~m}, 1 \mathrm{H}) .{ }^{13} \mathrm{C} \mathrm{NMR}\left(\mathrm{CDCl}_{3}\right): \delta 141.7$, 141.2, 136.7, 129.1, 128.9, 127.5, 127.4, 126.9, 125.8, 82.0, 70.8, 24.7. HRMS (FAB+): Calcd. for $\mathrm{C}_{15} \mathrm{H}_{12}[\mathrm{M}]$ 192.0939. Found 192.0938.

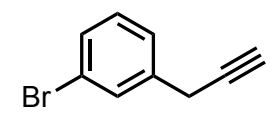

1-Bromo-3-(2-propyn-1-yl)benzene (4k) ${ }^{\mathrm{S} 24}$ : $41.4 \mathrm{mg}, 85 \%$ isolated yield. A pale yellow oil. ${ }^{1} \mathrm{H}$ NMR $\left(\mathrm{CDCl}_{3}\right): \delta 7.53-7.18(\mathrm{~m}, 4 \mathrm{H}), 3.59(\mathrm{~d}, J=2.5 \mathrm{~Hz}, 2 \mathrm{H}), 2.22(\mathrm{t}, J=2.5 \mathrm{~Hz}, 1 \mathrm{H}) .{ }^{13} \mathrm{C} \mathrm{NMR}\left(\mathrm{CDCl}_{3}\right): \delta$ $138.4,131.1,130.2,130.0,126.7,122.8,81.1,71.3,24.6$.

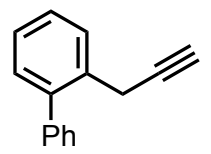

2-(2-Propyn-1-yl)-1,1'-biphenyl (4l) ${ }^{\mathrm{S} 25}: 45.6 \mathrm{mg}, 95 \%$ isolated yield (98\% NMR yield). A pale yellow oil. ${ }^{1} \mathrm{H}$ NMR $\left(\mathrm{CDCl}_{3}\right): \delta$ 7.68-7.66 (m, 1H), 7.47-7.28 (m, 8H), $3.52(\mathrm{~d}, J=2.7 \mathrm{~Hz}, 2 \mathrm{H}), 2.17(\mathrm{t}, J=2.7$ $\mathrm{Hz}, 1 \mathrm{H}) .{ }^{13} \mathrm{C} \mathrm{NMR}\left(\mathrm{CDCl}_{3}\right): \delta 141.5,141.0,133.8,130.1,129.3,129.0,128.4,127.9,127.3,127.0,82.8$, $70.5,22.9$. 


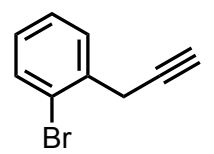

1-Bromo-2-(2-propyn-1-yl)benzene (4m) ${ }^{\mathrm{S} 26}: 41.1 \mathrm{mg}, 84 \%$ isolated yield (88\% NMR yield). A pale yellow oil. ${ }^{1} \mathrm{H}$ NMR $\left(\mathrm{CDCl}_{3}\right)$ : $\delta$ 7.64-7.53 $(\mathrm{m}, 2 \mathrm{H}), 7.34-7.30(\mathrm{~m}, 1 \mathrm{H}), 7.15-7.11(\mathrm{~m}, 1 \mathrm{H}), 3.68(\mathrm{~d}, J=$ $2.8 \mathrm{~Hz}, 2 \mathrm{H}), 2.26(\mathrm{t}, J=2.8 \mathrm{~Hz}, 1 \mathrm{H}) .{ }^{13} \mathrm{C} \mathrm{NMR}\left(\mathrm{CDCl}_{3}\right): \delta 135.6,132.7,129.8,128.6,127.8,123.9$, $80.8,71.7,26.0$.

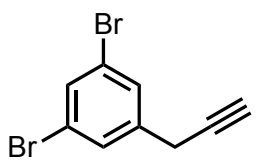

1,3-Dibromo-5-(2-propyn-1-yl)benzene (4n): $43.1 \mathrm{mg}, 63 \%$ isolated yield. A pale yellow solid, m.p. $=49.8-50.7{ }^{\circ} \mathrm{C} .{ }^{1} \mathrm{H}$ NMR $\left(\mathrm{CDCl}_{3}\right): \delta 7.56-7.55(\mathrm{~m}, 1 \mathrm{H}), 7.45-7.44(\mathrm{~m}, 2 \mathrm{H}), 3.57(\mathrm{~d}, J=2.6 \mathrm{~Hz}, 2 \mathrm{H})$, 2.26, (t, $J=2.6 \mathrm{~Hz}, 1 \mathrm{H}) .{ }^{13} \mathrm{C}$ NMR $\left(\mathrm{CDCl}_{3}\right): \delta 140.0,132.7,129.9,123.1,80.2,72.0,23.8$. HRMS (FAB+): Calcd for $\mathrm{C}_{9} \mathrm{H}_{6} \mathrm{Br}_{2}[\mathrm{M}]$ 273.8816. Found 273.8829.

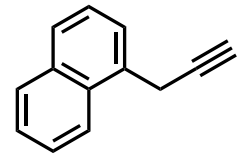

1-(2-Propyn-1-yl)naphthalene (4o) ${ }^{\mathrm{S} 19}: 38.2 \mathrm{mg}, 92 \%$ isolated yield. A pale yellow oil. ${ }^{1} \mathrm{H}$ NMR $\left(\mathrm{CDCl}_{3}\right): \delta 8.04-8.02(\mathrm{~m}, 1 \mathrm{H}), 7.90-7.88(\mathrm{~m}, 1 \mathrm{H}), 7.81-7.79(\mathrm{~m}, 1 \mathrm{H}), 7.67-7.65(\mathrm{~m}, 1 \mathrm{H}), 7.59-7.45(\mathrm{~m}$, $3 \mathrm{H}), 4.03(\mathrm{~d}, J=2.8 \mathrm{~Hz}, 2 \mathrm{H}), 2.28(\mathrm{t}, J=2.8 \mathrm{~Hz}, 1 \mathrm{H}) .{ }^{13} \mathrm{C} \mathrm{NMR}\left(\mathrm{CDCl}_{3}\right): \delta 133.8,132.0,131.4,128.9$, $127.8,126.4,125.9,125.8,125.7,123.4,81.8,71.5,22.9$.

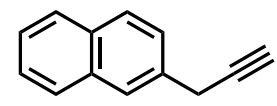

2-(2-Propyn-1-yl)naphthalene (4p) ${ }^{\mathrm{S} 19}: 39.0 \mathrm{mg}$, 94\% isolated yield. A pale yellow solid. ${ }^{1} \mathrm{H}$ NMR $\left(\mathrm{CDCl}_{3}\right): \delta 7.84-7.81(\mathrm{~m}, 4 \mathrm{H}), 7.51-7.44(\mathrm{~m}, 3 \mathrm{H}), 3.79(\mathrm{~d}, J=2.0 \mathrm{~Hz}, 2 \mathrm{H}), 2.28-2.27(\mathrm{~m}, 1 \mathrm{H}) .{ }^{13} \mathrm{C}$ NMR $\left(\mathrm{CDCl}_{3}\right): \delta 133.6,132.5,128.4,127.8,126.4,126.3,125.8,82.0,70.9,25.1$.

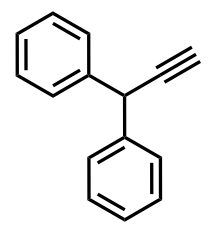

3,3-Diphenyl-1-propyne (4q) ${ }^{\mathrm{S} 27}: 35.6 \mathrm{mg}, 74 \%$ isolated yield. A pale yellow oil. ${ }^{1} \mathrm{H}$ NMR $\left(\mathrm{CDCl}_{3}\right): \delta$ 7.39-7.21 (m, 10H), $5.00(\mathrm{~d}, J=2.0 \mathrm{~Hz}, 1 \mathrm{H}), 2.49-2.48(\mathrm{~m}, 1 \mathrm{H}) .{ }^{13} \mathrm{C} \mathrm{NMR}\left(\mathrm{CDCl}_{3}\right): \delta 141.2,128.8$, $128.0,127.1,84.7,73.0,43.0$. 


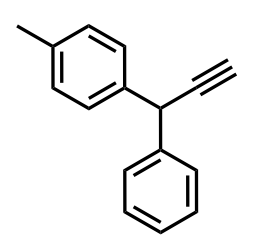

3-Phenyl-3-(4-tolyl)-1-propyne (4r) ${ }^{\mathrm{S} 28}: 39.7 \mathrm{mg}$, 77\% isolated yield. A pale yellow oil. ${ }^{1} \mathrm{H}$ NMR $\left(\mathrm{CDCl}_{3}\right): \delta$ 7.40-7.38 (m, 2H), 7.33-7.21 (m, 5H), 7.14-7.12 (m, 2H), 4.99 (d, J=2.2 Hz, 1H), 2.49 (d, $J=2.2 \mathrm{~Hz}, 1 \mathrm{H}), 2.32(\mathrm{~s}, 3 \mathrm{H}) .{ }^{13} \mathrm{C} \mathrm{NMR}\left(\mathrm{CDCl}_{3}\right): \delta 141.4,138.3,136.8,129.5,128.8,127.9,127.8$, 127.1, 84.9, 72.8, 42.6, 21.3.

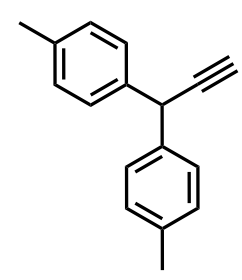

3,3-Bis(4-tolyl)-1-propyne (4s): $44.0 \mathrm{mg}, 80 \%$ isolated yield. A pale yellow oil. ${ }^{1} \mathrm{H} \mathrm{NMR}\left(\mathrm{CDCl}_{3}\right): \delta$ 7.30-7.28 (m, 4H), 7.15-7.13 (m, 4H), 4.97 (d, $J=2.4 \mathrm{~Hz}, 1 \mathrm{H}), 2.49$ (d, $J=2.4 \mathrm{~Hz}, 1 \mathrm{H}), 2.336(\mathrm{~s}, 6 \mathrm{H})$. ${ }^{13} \mathrm{C}$ NMR $\left(\mathrm{CDCl}_{3}\right): \delta 138.5,136.7,129.4,127.8,85.1,72.6,42.3,21.0$. HRMS (FAB+): Calcd. for $\mathrm{C}_{17} \mathrm{H}_{16}[\mathrm{M}] 220.1252$. Found 220.1255.

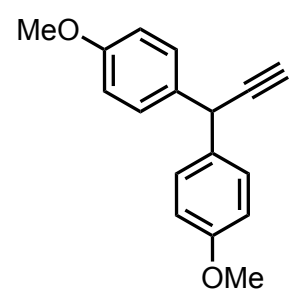

3,3-Bis(4-methoxyphenyl)-1-propyne (4t) ${ }^{\mathrm{S} 29}: 41.6 \mathrm{mg}, 66 \%$ isolated yield. A yellow solid. ${ }^{1} \mathrm{H}$ NMR $\left(\mathrm{CDCl}_{3}\right): \delta 7.29-7.25(\mathrm{~m}, 4 \mathrm{H}), 6.86-6.82(\mathrm{~m}, 4 \mathrm{H}), 4.92(\mathrm{~d}, J=2.6 \mathrm{~Hz}, 1 \mathrm{H}), 3.77(\mathrm{~s}, 6 \mathrm{H}), 2.47(\mathrm{~d}, J=2.6$ $\mathrm{Hz}, 1 \mathrm{H}) .{ }^{13} \mathrm{C} \mathrm{NMR}\left(\mathrm{CDCl}_{3}\right): \delta 158.6,133.7,128.8,114.1,85.3,72.6,55.4,41.4$.

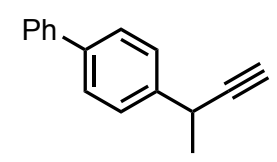

4-(3-Butyn-2-yl)-1,1'-biphenyl (4u) ${ }^{\mathrm{S} 30}: 45.4 \mathrm{mg}, 88 \%$ isolated yield. A pale yellow solid. ${ }^{1} \mathrm{H}$ NMR $\left(\mathrm{CDCl}_{3}\right): \delta$ 7.59-7.55 (m, 4H), 7.48-7.42 (m, 4H), 7.36-7.32 (m, 1H), 3.85-3.79 (m, 1H), $2.29(\mathrm{~d}, J=2.8$ $\mathrm{Hz}, 1 \mathrm{H}), 1.54(\mathrm{~s}, 3 \mathrm{H}) .{ }^{13} \mathrm{C} \mathrm{NMR}\left(\mathrm{CDCl}_{3}\right): \delta 141.9,141.0,139.9,128.9,127.5,127.4,127.3,127.2,87.2$, 70.4, 31.8, 24.6. 
4. Large-Scale Reaction of Ruthenium-Catalyzed Propargylic Reduction of Propargylic Alcohols (2a) with Hantzsch Ester

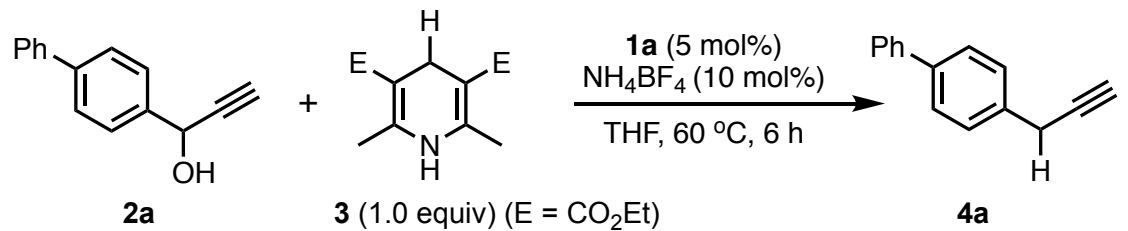

A typical experiment procedure for the reaction of 1-(1,1'-biphenyl-4-yl)-2-propyn-1-ol (2a) with Hantzsch ester $\mathbf{3}$ is described below. In a $20 \mathrm{~mL}$ Schlenk flask were placed ruthenium catalyst 1a (32.0 $\mathrm{mg}, 0.050 \mathrm{mmol})$ and $\mathrm{NH}_{4} \mathrm{BF}_{4}(10.4 \mathrm{mg}, 0.100 \mathrm{mmol})$ under $\mathrm{N}_{2}$. Anhydrous THF (10.0 mL) was added, and then $2 \mathrm{a}(208.0 \mathrm{mg}, 1.00 \mathrm{mmol})$ and $\mathbf{3}(253.2 \mathrm{mg}, 1.00 \mathrm{mmol})$ were added. The mixture was stirred at $60{ }^{\circ} \mathrm{C}$ for 6 hours. The solvent was removed under reduced pressure, and the residue was purified by the column chromatography $\left(\mathrm{SiO}_{2}\right)$ with hexane/EtOAc (99:1) as eluent to give 4-(2-propynyl)-1,1'biphenyl (4a) ${ }^{\mathrm{S} 19}$ as a pale yellow solid (189.5 $\mathrm{mg}, 0.99 \mathrm{mmol}, 99 \%$ isolated yield). 


\section{Computational Details}

DFT calculations were carried out with the Gaussian16 (Revision A.03) program package. ${ }^{\mathrm{S} 1}$ Geometry optimization and analytical vibrational frequency analysis were performed by the restricted Kohn-Sham $\mathrm{DFT}^{\mathrm{S} 32}$ using the long-range-corrected functional including the empirical dispersion $\left(\omega \mathrm{B} 97 \mathrm{XD}\right.$ method). ${ }^{\mathrm{S} 33}$ In the numerical integration, a larger grid (superfinegrid) was used. ${ }^{\mathrm{S} 31}$ Pople's 6-31G $(d)$ basis set ${ }^{\mathrm{S} 34}$ for $\mathrm{C}, \mathrm{H}, \mathrm{N}, \mathrm{O}, \mathrm{S}$, and $\mathrm{Cl}$ atoms (6d-type) and the SDD basis set ${ }^{\mathrm{S} 35}$ with the effective core potential for Ru atom were used for the Gaussian basis functions. Solvent effect of THF was taken into account by the IEF-PCM method ( $\left(\mathrm{B} 97 \mathrm{XD}\right.$ (IEFPCM)/(SDD, 6-31G*)). ${ }^{\mathrm{S3}} \quad$ For the evaluation of the entropy of each optimized structure, the quasi-harmonic treatment was applied. ${ }^{\mathrm{S} 7, \mathrm{~S} 38}$ The energies of each structure were calculated by using larger basis set (Pople's 6-311++G $(d, p)$ basis set ${ }^{\mathrm{S} 34}$ for $\mathrm{C}, \mathrm{H}, \mathrm{N}, \mathrm{O}, \mathrm{S}$, and $\mathrm{Cl}$ atoms (5d-type) and the $\mathrm{SDD}$ basis set for $\mathrm{Ru}$ atom; $\left.\omega B 97 X D(I E F P C M) /\left(\operatorname{SDD}, 6-311++G^{* *}\right) / / \omega B 97 X D(I E F P C M) /\left(S D D, 6-31 G^{*}\right)\right)$.

Total electronic energy $E$ and Gibbs free energy $G^{298 \mathrm{~K}}$ are shown in Table S1, and the Gibbs free energy diagram is represented in Figure S1, which corresponds to Figure 1. Each optimized structure is shown in Figure S2. We obtained three structures for the Ruallenylidene complex, $\mathbf{I}_{\mathbf{A}}, \mathbf{I}_{\mathbf{B}}$, and $\mathbf{I}_{\mathbf{C}}$, which have almost the same Gibbs free energies (the differences are within $0.2 \mathrm{kcal} / \mathrm{mol}$ ). For the transition state TS III-IV, the changes in bond distances and fragment charges along the IRC are drawn in Figures S3 and S4, respectively. As shown in Figure S4, the charge of the hydrogen atom is invariant, while the charges in the Ru-allenylidene and ester fragments become altered through the transition state. As a result, the hydrogen atom transfers formally as a hydride. 
Table S1. Total Electronic Energy $E$ and Gibbs Free Energy $G^{298 \mathrm{~K}}(\mathrm{au})$.

\begin{tabular}{|c|c|c|c|c|c|}
\hline & \multicolumn{3}{|c|}{$\omega \mathrm{B} 97 \mathrm{XD}(\mathrm{IEFPCM}) /\left(\mathrm{SDD}, 6-31 \mathrm{G}^{*}\right)$} & \multicolumn{2}{|c|}{$\begin{array}{c}\omega \mathrm{B} 97 \mathrm{XD}(\mathrm{IEFPCM}) /\left(\mathrm{SDD}, 6-311++\mathrm{G}^{* *}\right) \\
/ / \omega \mathrm{B} 97 \mathrm{XD}(\mathrm{IEFPCM}) /\left(\mathrm{SDD}, 6-31 \mathrm{G}^{*}\right)\end{array}$} \\
\hline & $E$ & enthalpy term & $T S$ & $E$ & $G^{298 \mathrm{~K}}$ \\
\hline $\mathbf{I}_{\mathbf{A}}$ & -2652.624888 & 0.702604 & 0.111787 & -2652.985514 & -2652.394697 \\
\hline $\mathbf{I}_{\mathbf{B}}$ & -2652.623899 & 0.702458 & 0.112356 & -2652.984435 & -2652.394333 \\
\hline $\mathbf{I}_{\mathbf{C}}$ & -2652.623726 & 0.702404 & 0.112610 & -2652.984306 & -2652.394511 \\
\hline II & -862.247487 & 0.334715 & 0.066737 & -862.477904 & -862.209926 \\
\hline III & -3514.899469 & 1.040096 & 0.152301 & -3515.487795 & -3514.600001 \\
\hline TS $_{\text {III-IV }}$ & -3514.886175 & 1.035870 & 0.149200 & -3515.474102 & -3514.587432 \\
\hline IV & -3514.925025 & 1.041212 & 0.152050 & -3515.513490 & -3514.624327 \\
\hline $\mathbf{V}$ & -2653.401244 & 0.713775 & 0.113544 & -2653.769519 & -2653.169287 \\
\hline VI & -861.493454 & 0.324666 & 0.065132 & -861.714598 & -861.455065 \\
\hline VII & -3514.927576 & 1.040553 & 0.152964 & -3515.516966 & -3514.629377 \\
\hline TS VIII-VIII & -3514.913806 & 1.034358 & 0.151749 & -3515.502249 & -3514.619640 \\
\hline VIII & -3514.942051 & 1.038785 & 0.151524 & -3515.527696 & 3514.640436 \\
\hline IX & -2653.859143 & 0.725929 & 0.112050 & -2654.223129 & -2653.609250 \\
\hline $\mathbf{X}$ & -861.053567 & 0.310156 & 0.066003 & -861.277245 & -861.0333092 \\
\hline
\end{tabular}




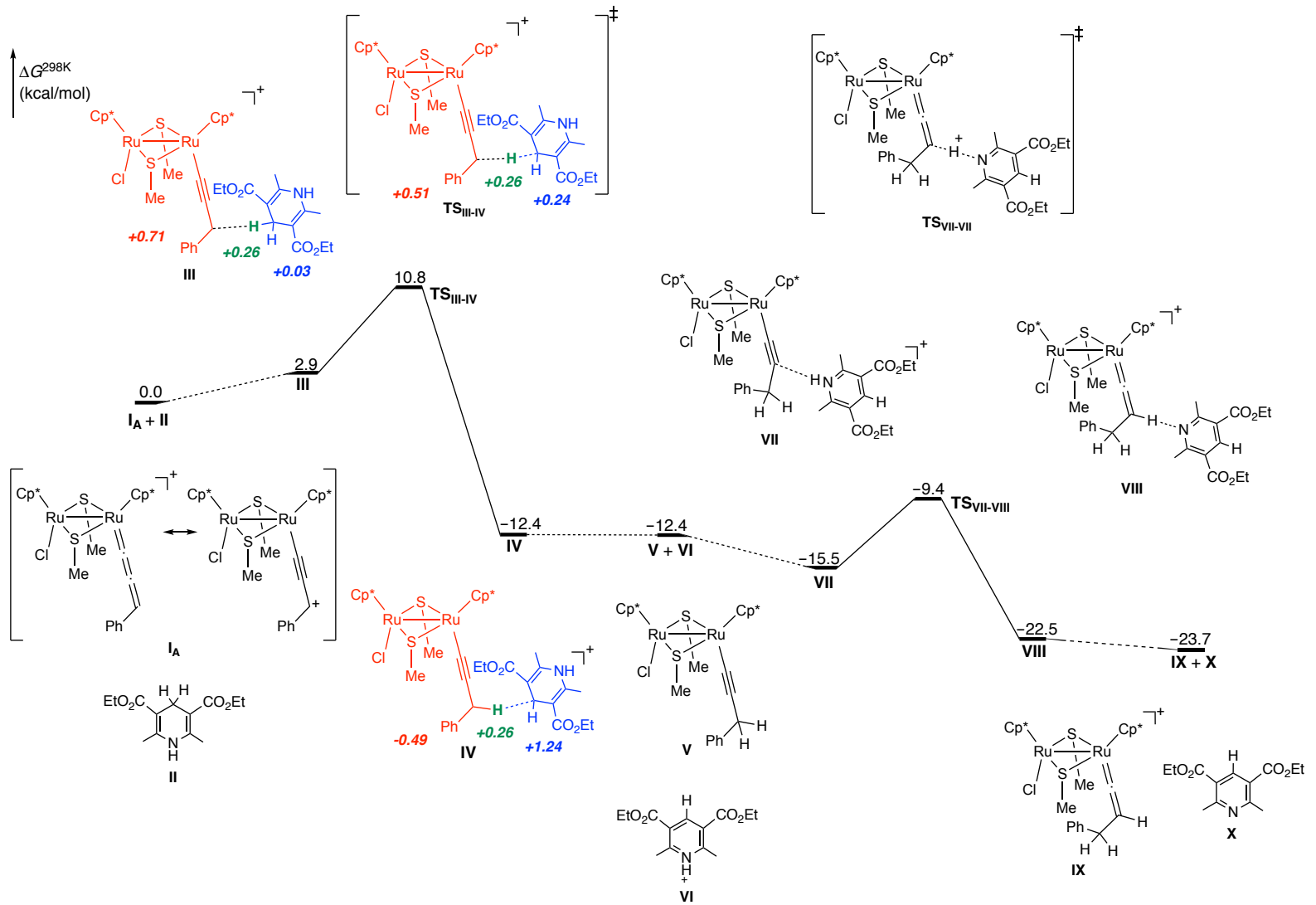

Figure S1. Gibbs free energy diagram (kcal/mol). 


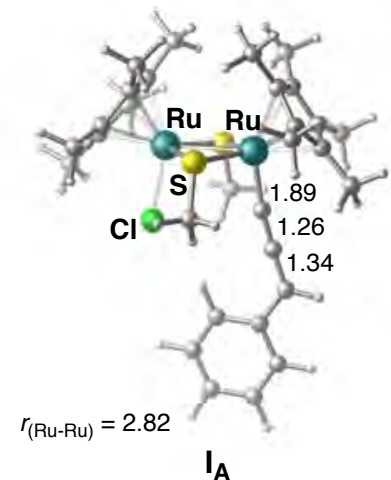

$\left(\Delta G^{298 \mathrm{~K}}=0.0 \mathrm{kcal} / \mathrm{mol}\right)$

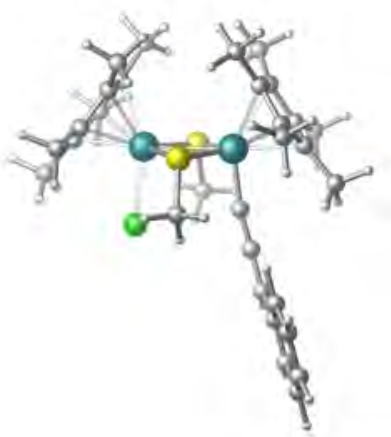

$I_{B}$

$\left(\Delta G^{298 \mathrm{~K}}=0.2 \mathrm{kcal} / \mathrm{mol}\right)$

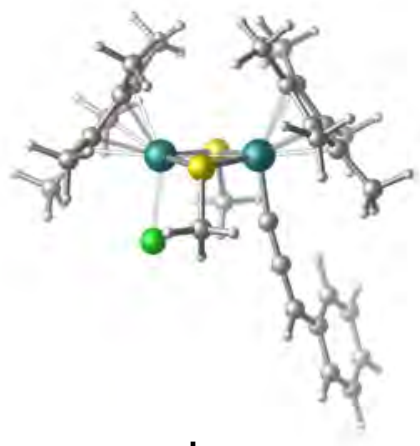

$I_{C}$

$\left(\Delta G^{298 \mathrm{~K}}=0.1 \mathrm{kcal} / \mathrm{mol}\right)$

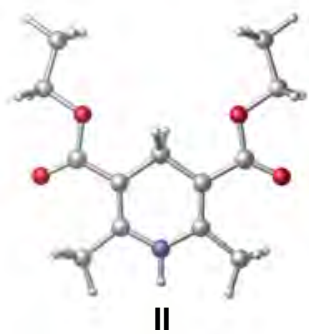

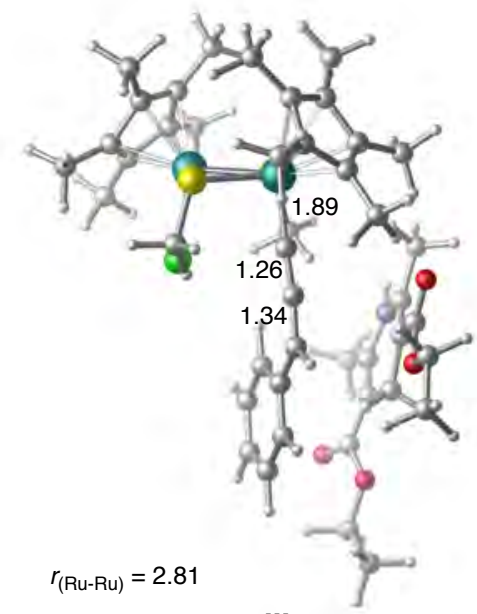

III

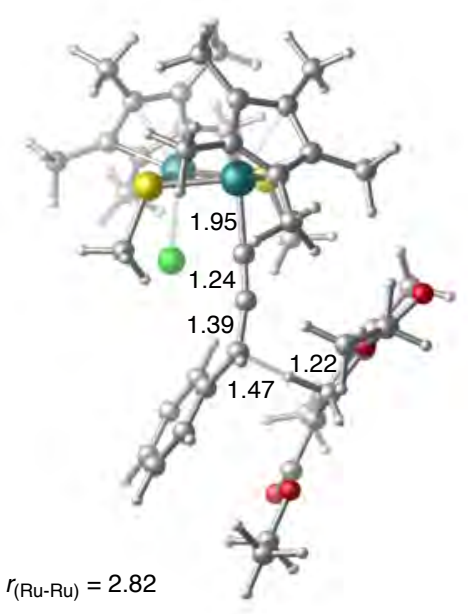

TS $_{\text {III-IV }}$
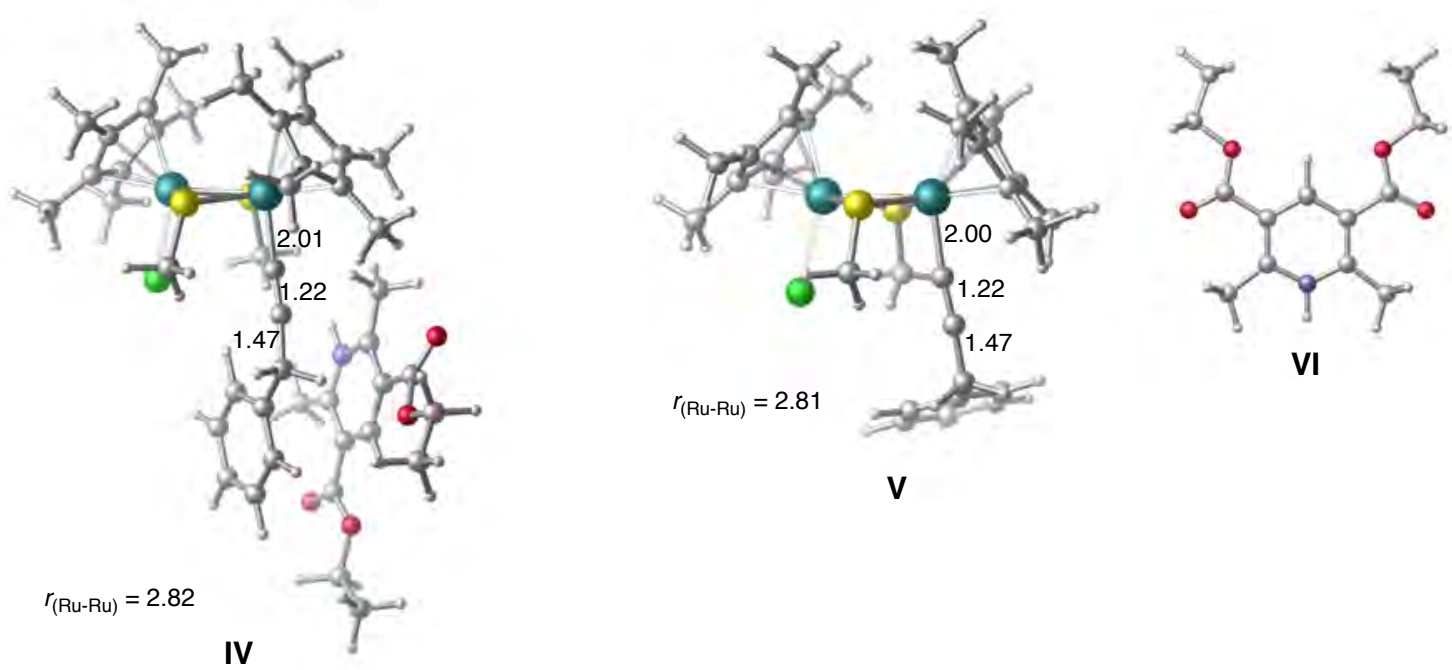

Figure S2. Structures optimized at the $\omega \mathrm{B} 97 \mathrm{XD}(\mathrm{IEFPCM}) /\left(\mathrm{SDD}, 6-31 \mathrm{G}^{*}\right)$ level of theory. Bond lengths are given in $\AA$. 

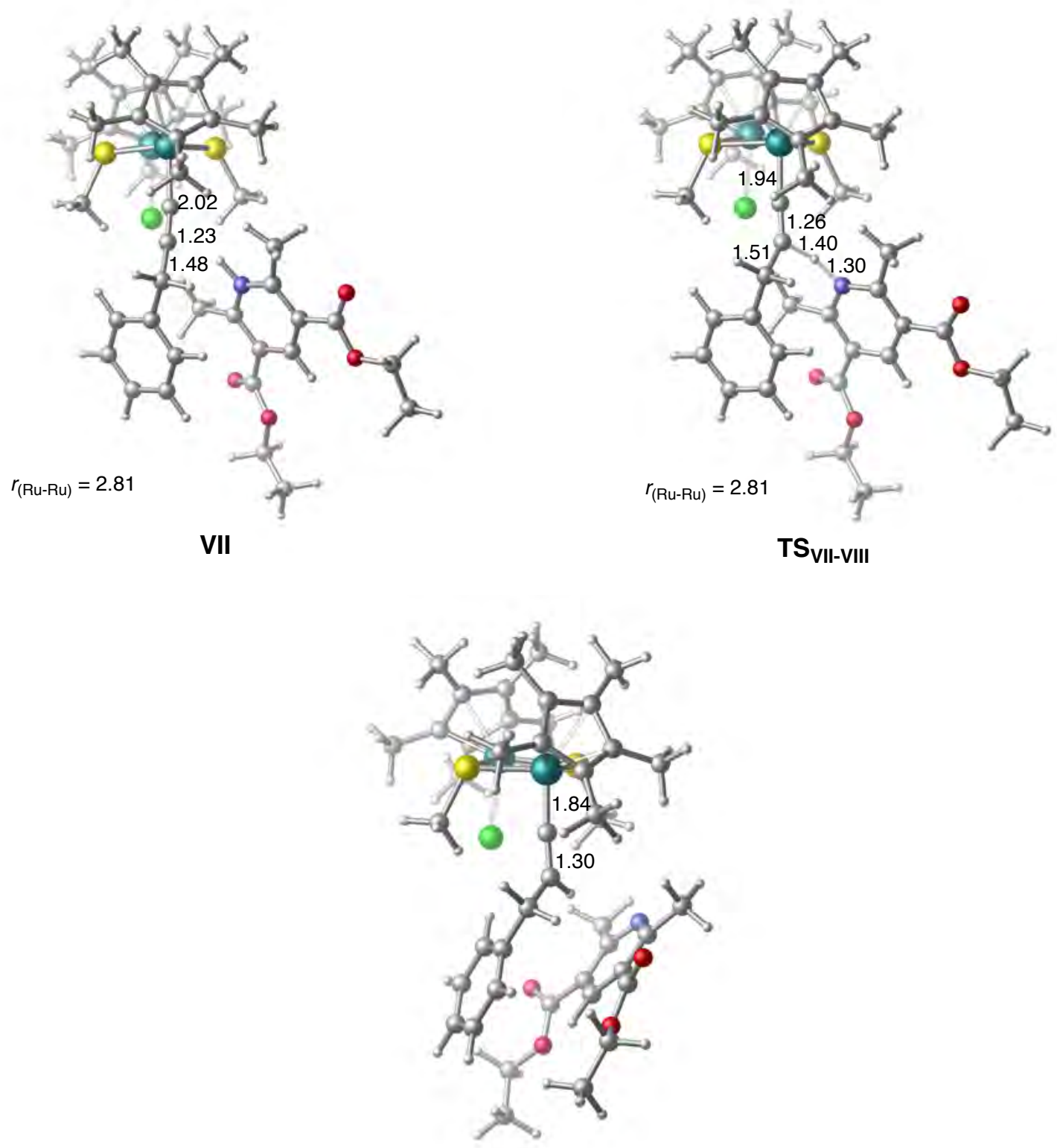

$r_{(\mathrm{Ru}-\mathrm{Ru})}=2.80 \quad$ VIII
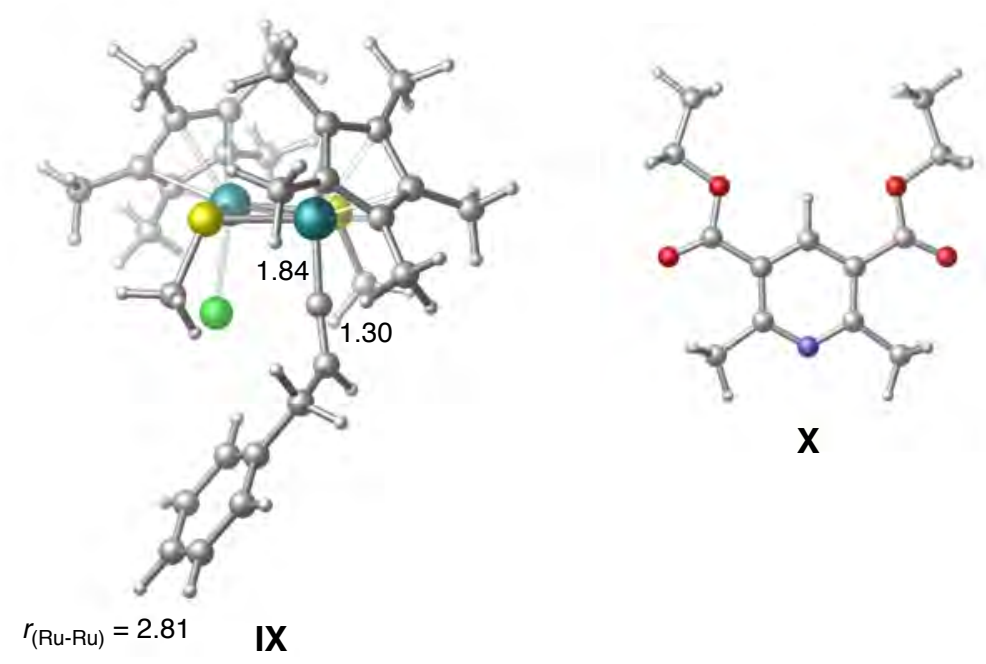

Figure S2. (Continued.) 

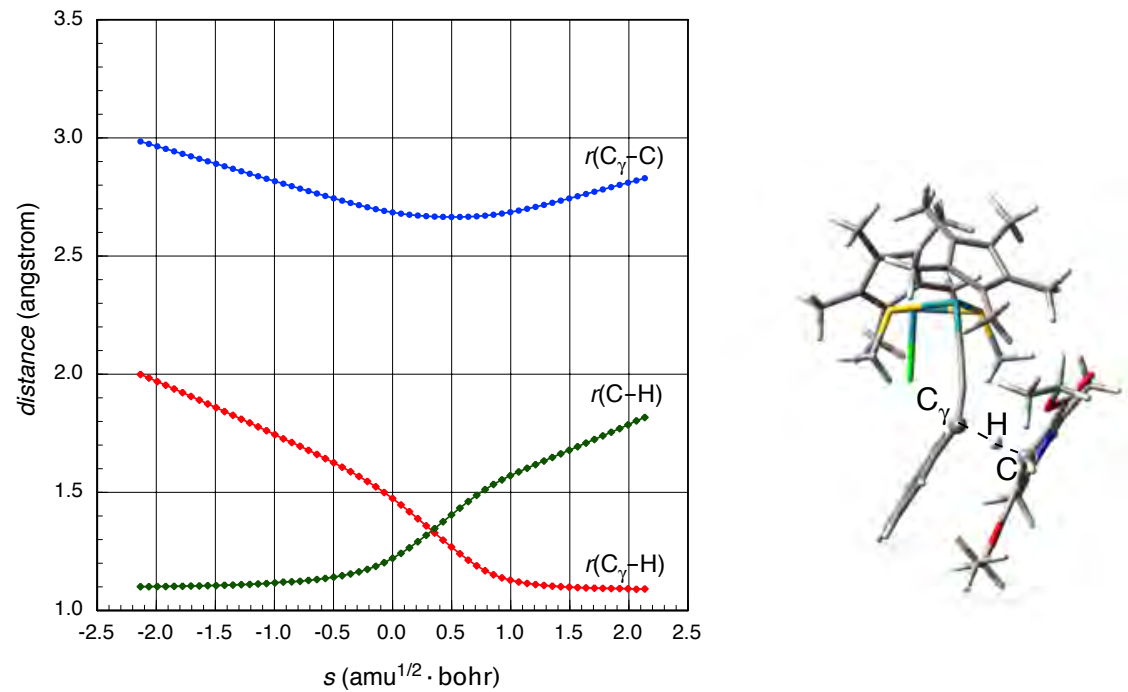

Figure S3. Changes in bond distances along the IRC of TS $\mathbf{T I I - I V}$.

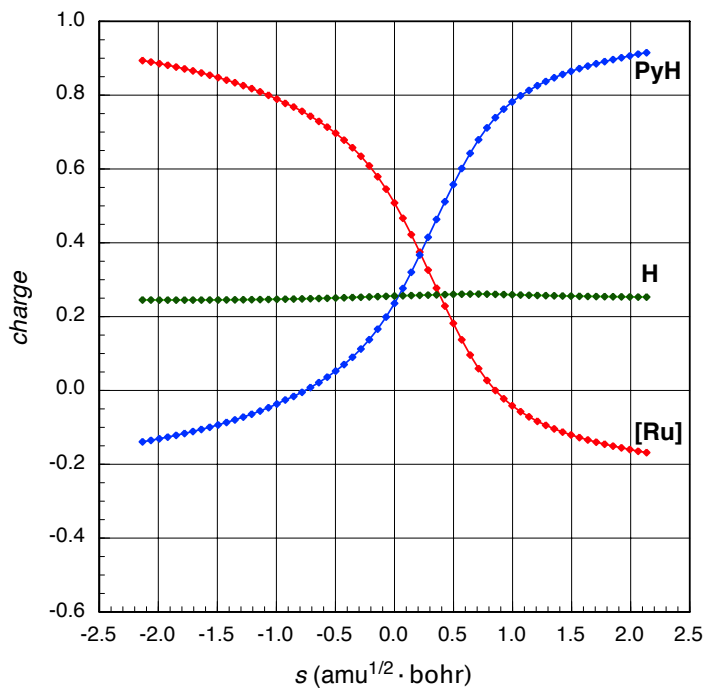

Figure S4. Changes in fragment charges along the IRC of TS III-IV. Charges are estimated by the natural population analysis (NPA). 
6. ${ }^{1} \mathrm{H},{ }^{13} \mathrm{C}$ NMR and ${ }^{19}$ F NMR spectra

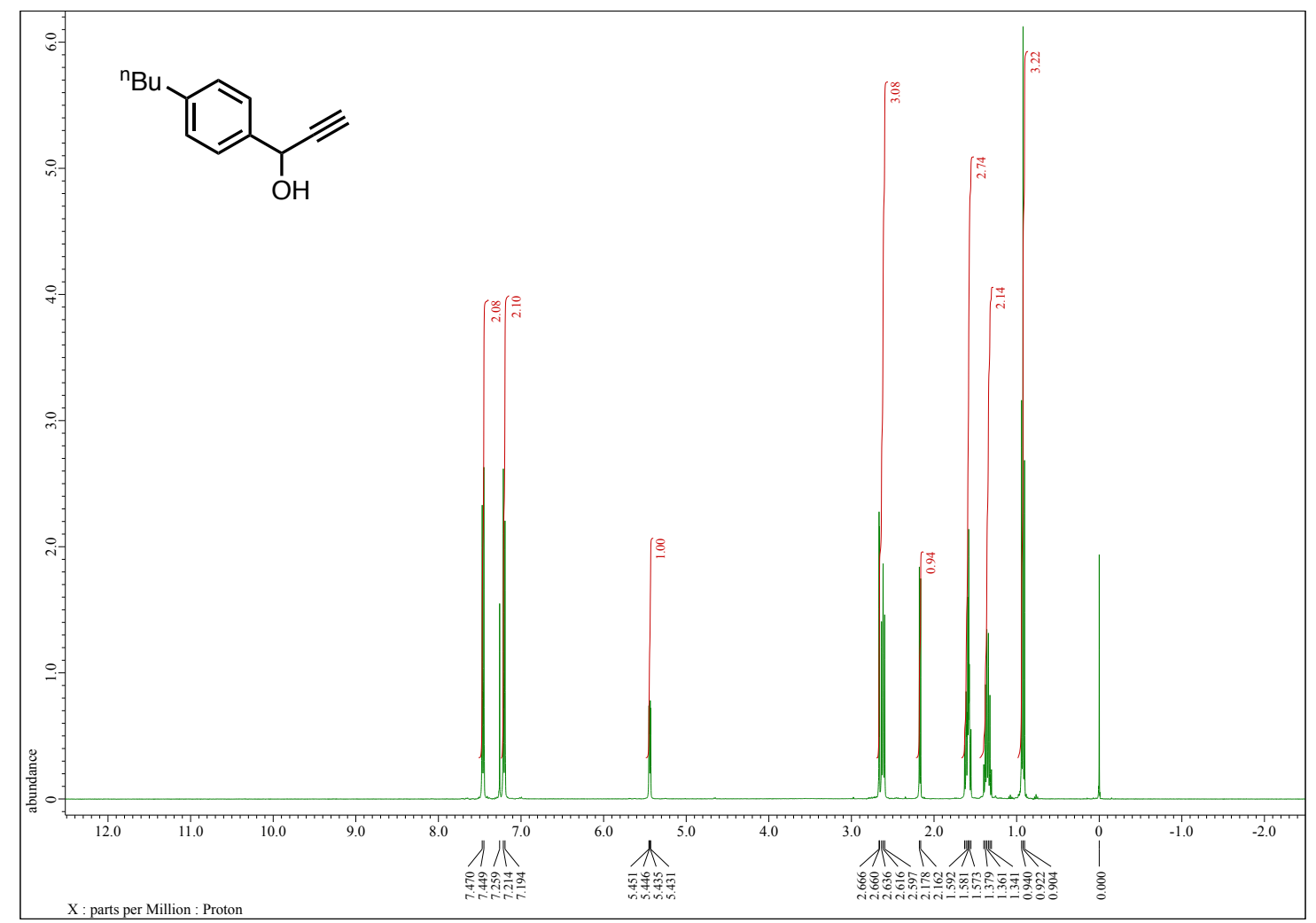

Figure S5. ${ }^{1} \mathrm{H}$ NMR of $\mathbf{2 d}\left(\mathrm{CDCl}_{3}\right)$.

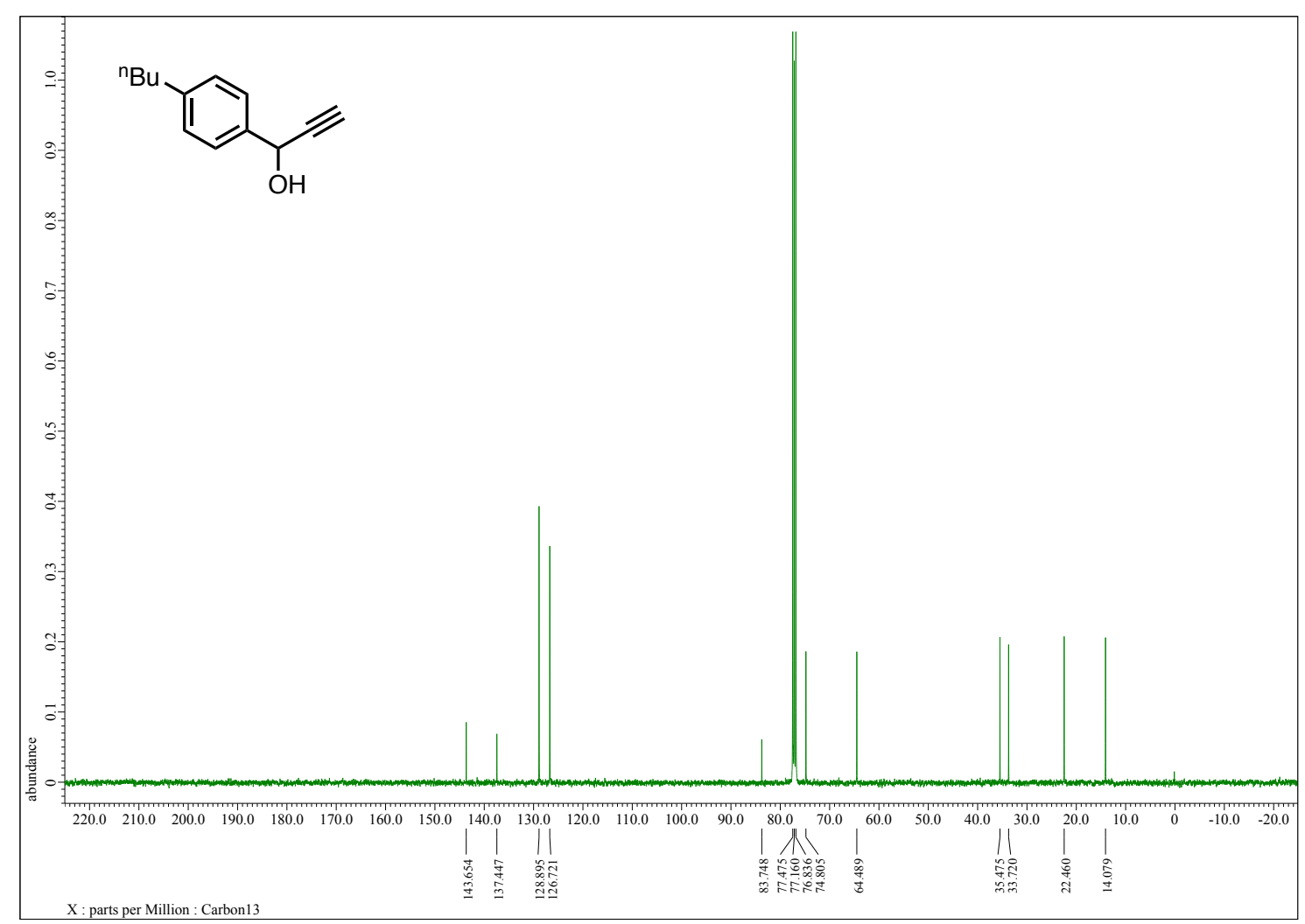

Figure S6. ${ }^{13} \mathrm{C} \mathrm{NMR}$ of $\mathbf{2 d}\left(\mathrm{CDCl}_{3}\right)$. 


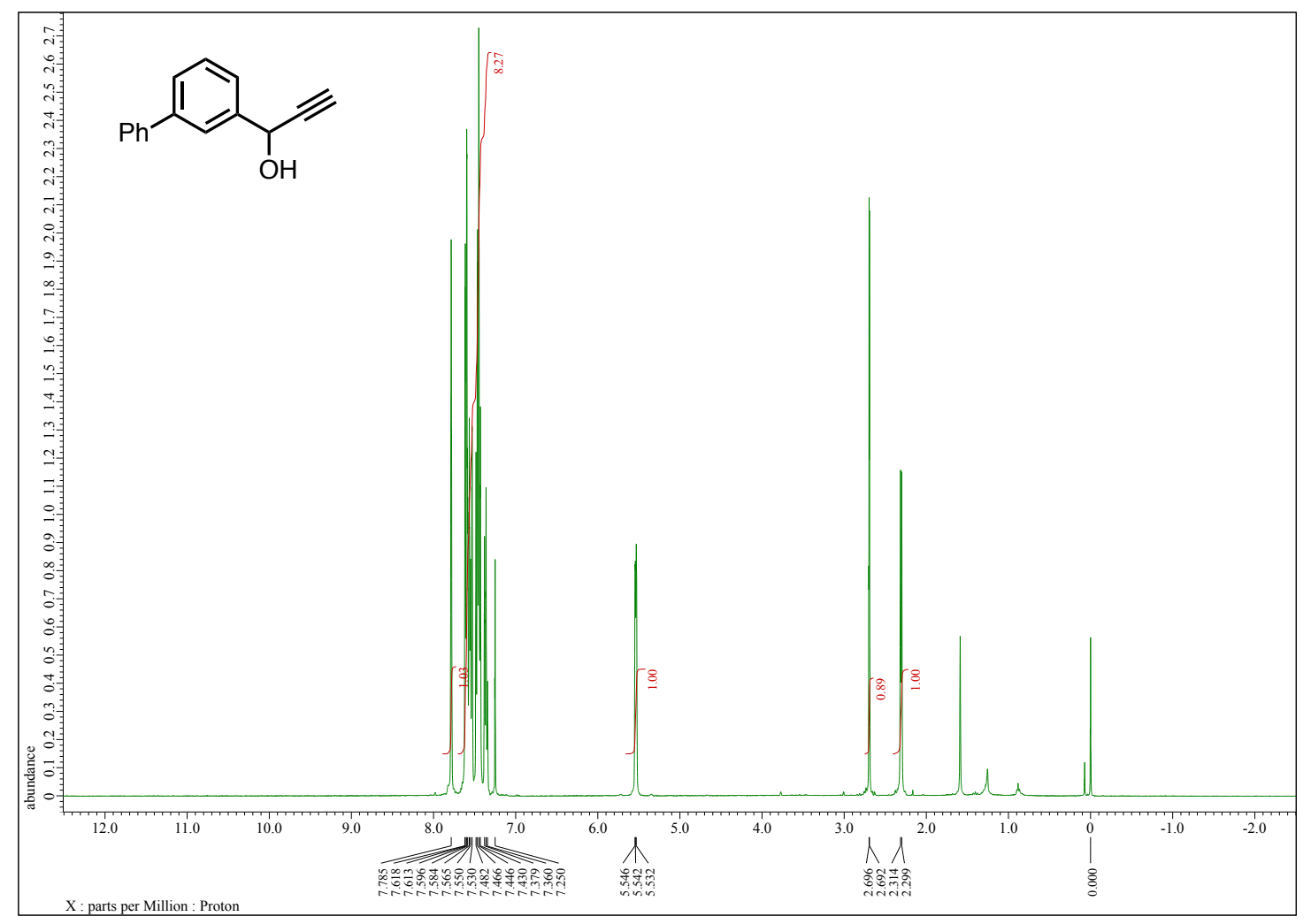

Figure S7. ${ }^{1} \mathrm{H} \mathrm{NMR}$ of $\mathbf{2} \mathbf{j}\left(\mathrm{CDCl}_{3}\right)$.

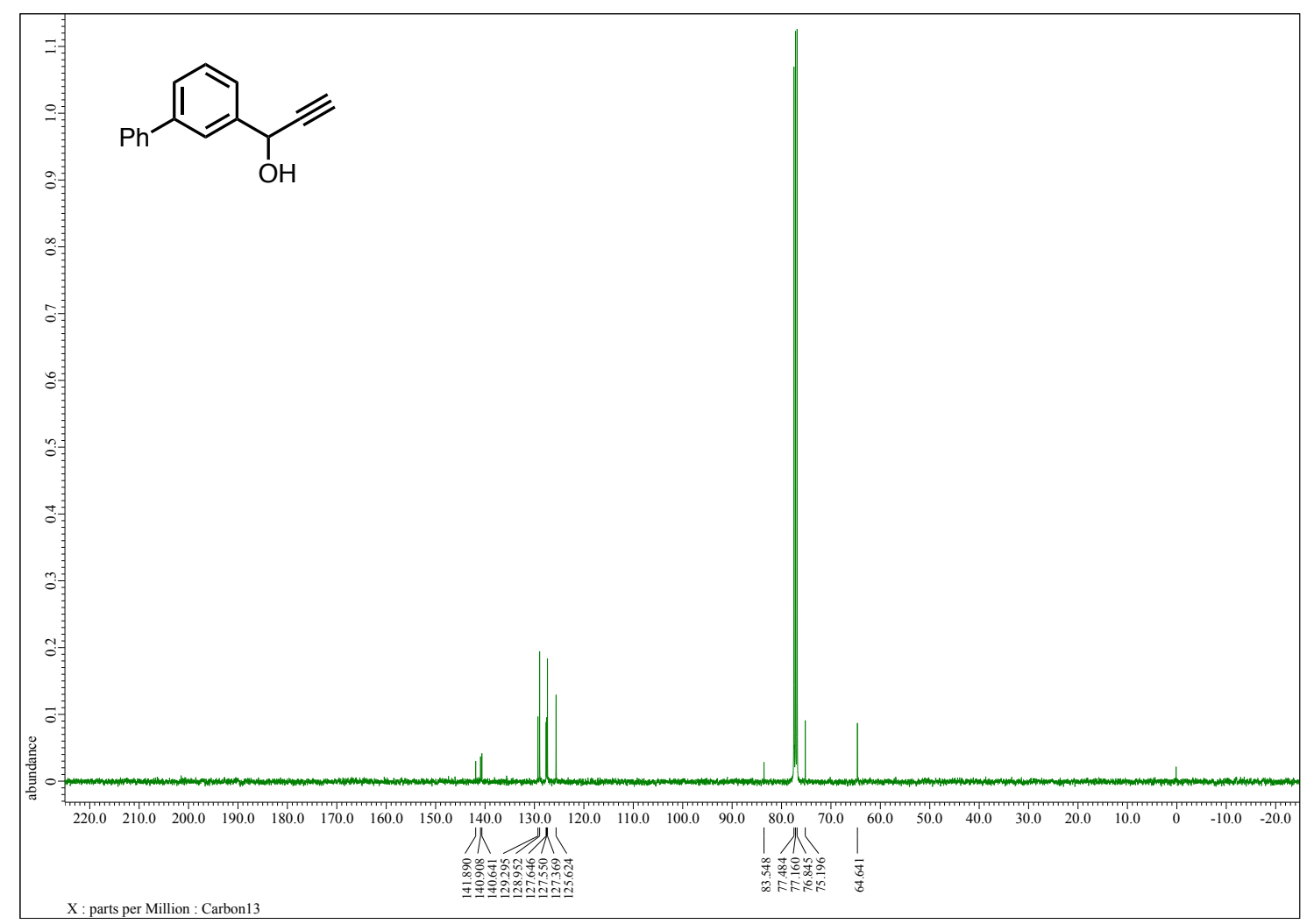

Figure S8. ${ }^{13} \mathrm{C}$ NMR of $\mathbf{2 j}\left(\mathrm{CDCl}_{3}\right)$. 


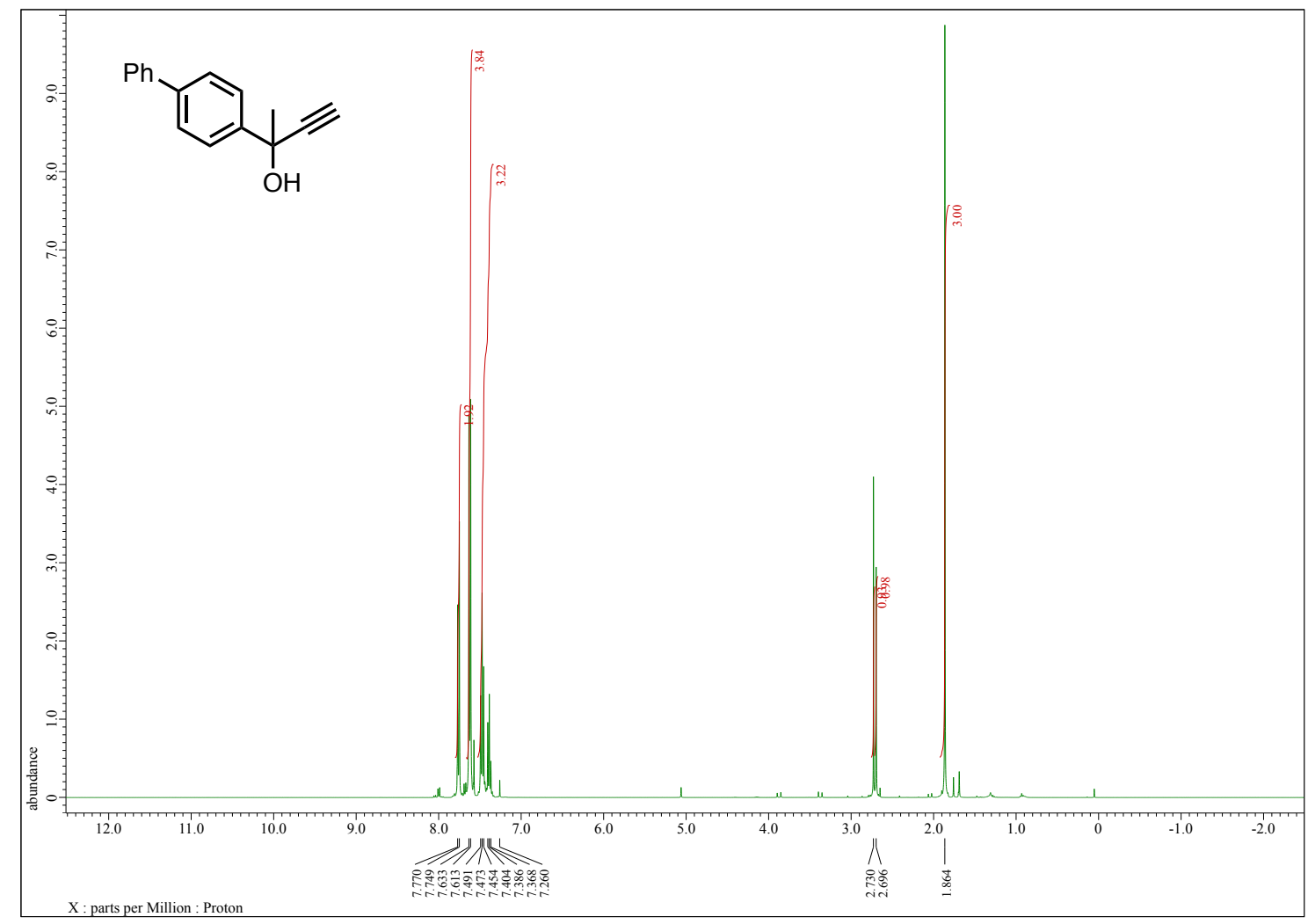

Figure S9. ${ }^{1} \mathrm{H} \mathrm{NMR}$ of $\mathbf{2 u}\left(\mathrm{CDCl}_{3}\right)$.

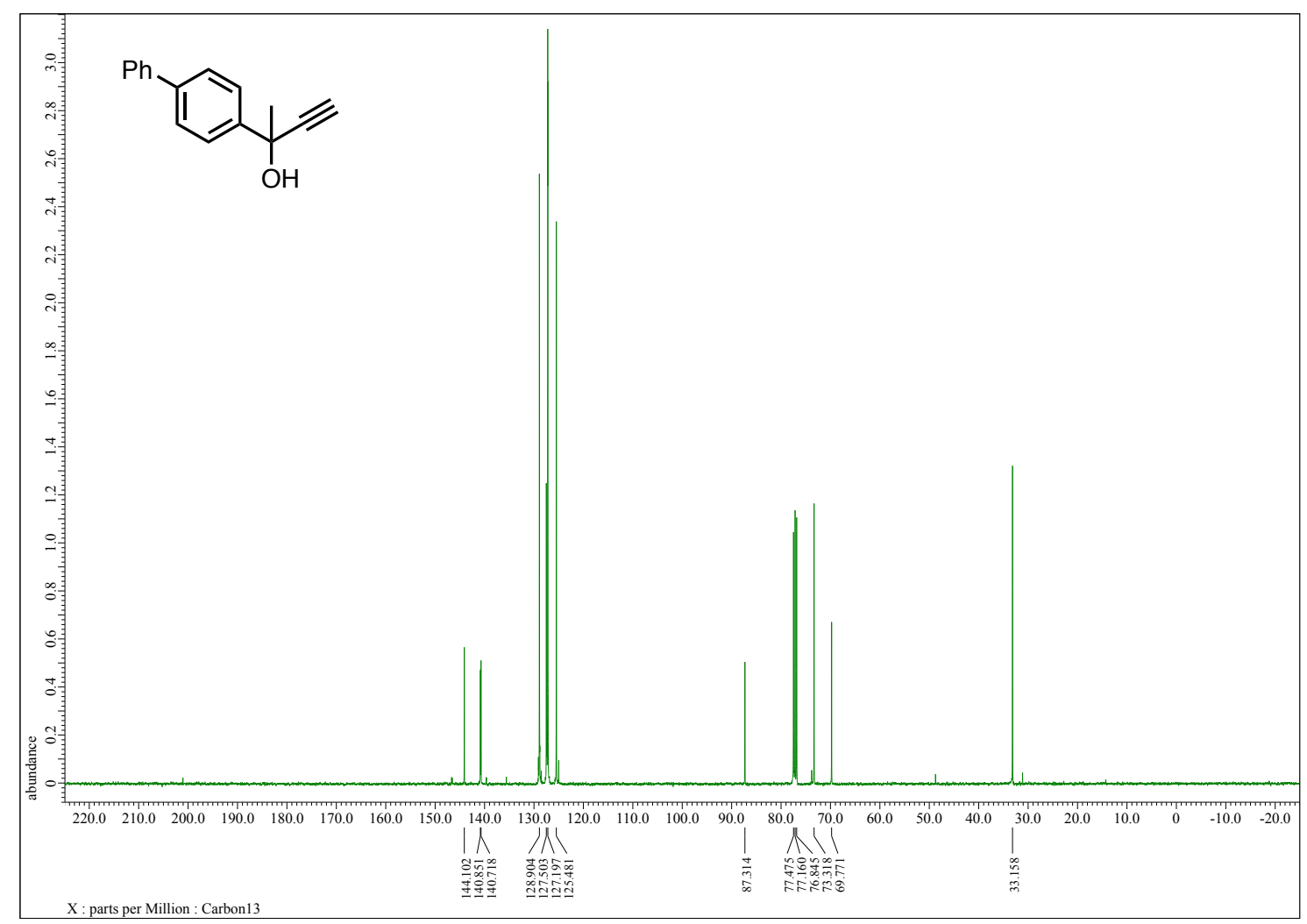

Figure S10. ${ }^{13} \mathrm{C} \mathrm{NMR} \mathrm{of} \mathbf{2 u}\left(\mathrm{CDCl}_{3}\right)$. 


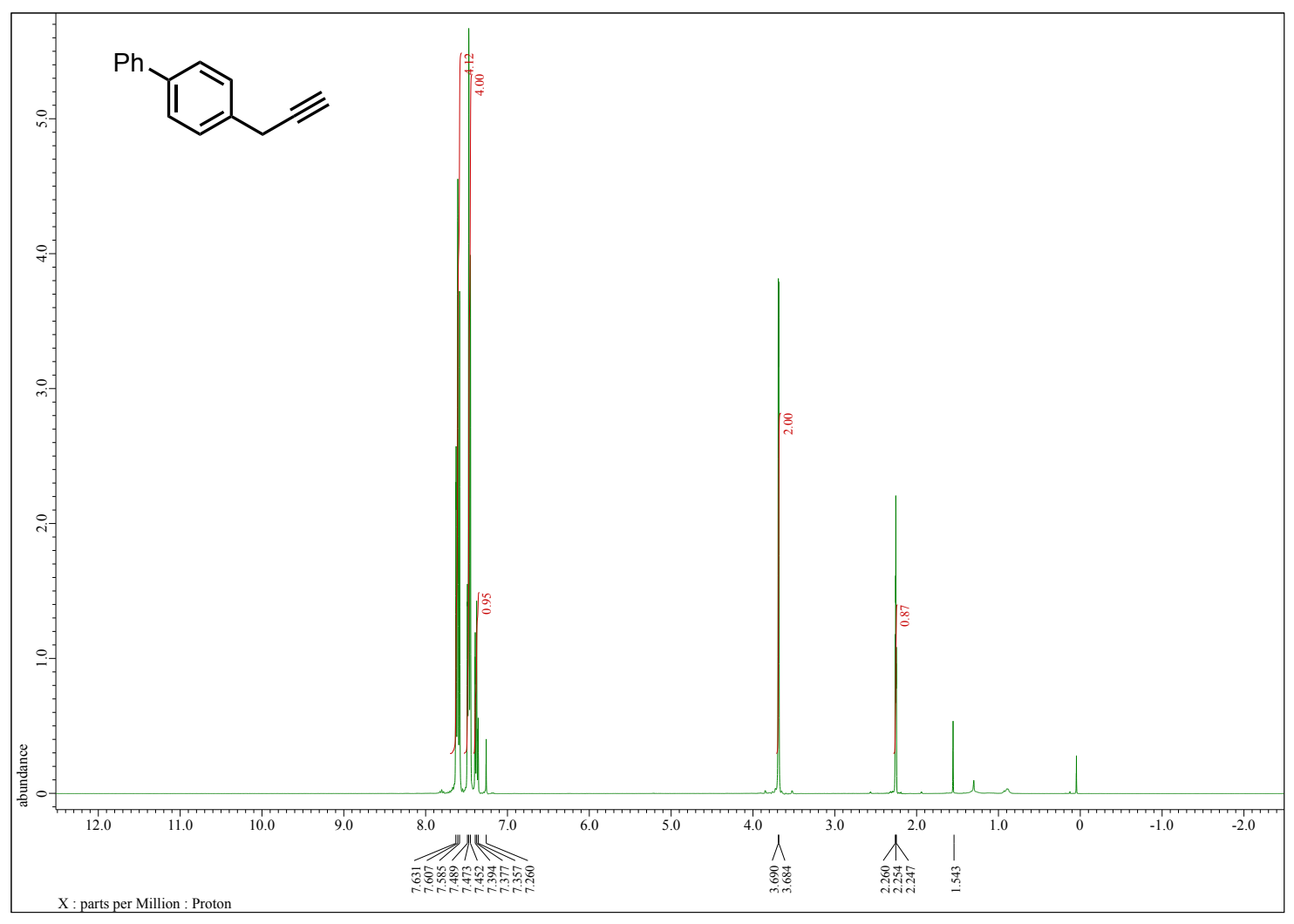

Figure S11. ${ }^{1} \mathrm{H}$ NMR of $\mathbf{4 a}\left(\mathrm{CDCl}_{3}\right)$.

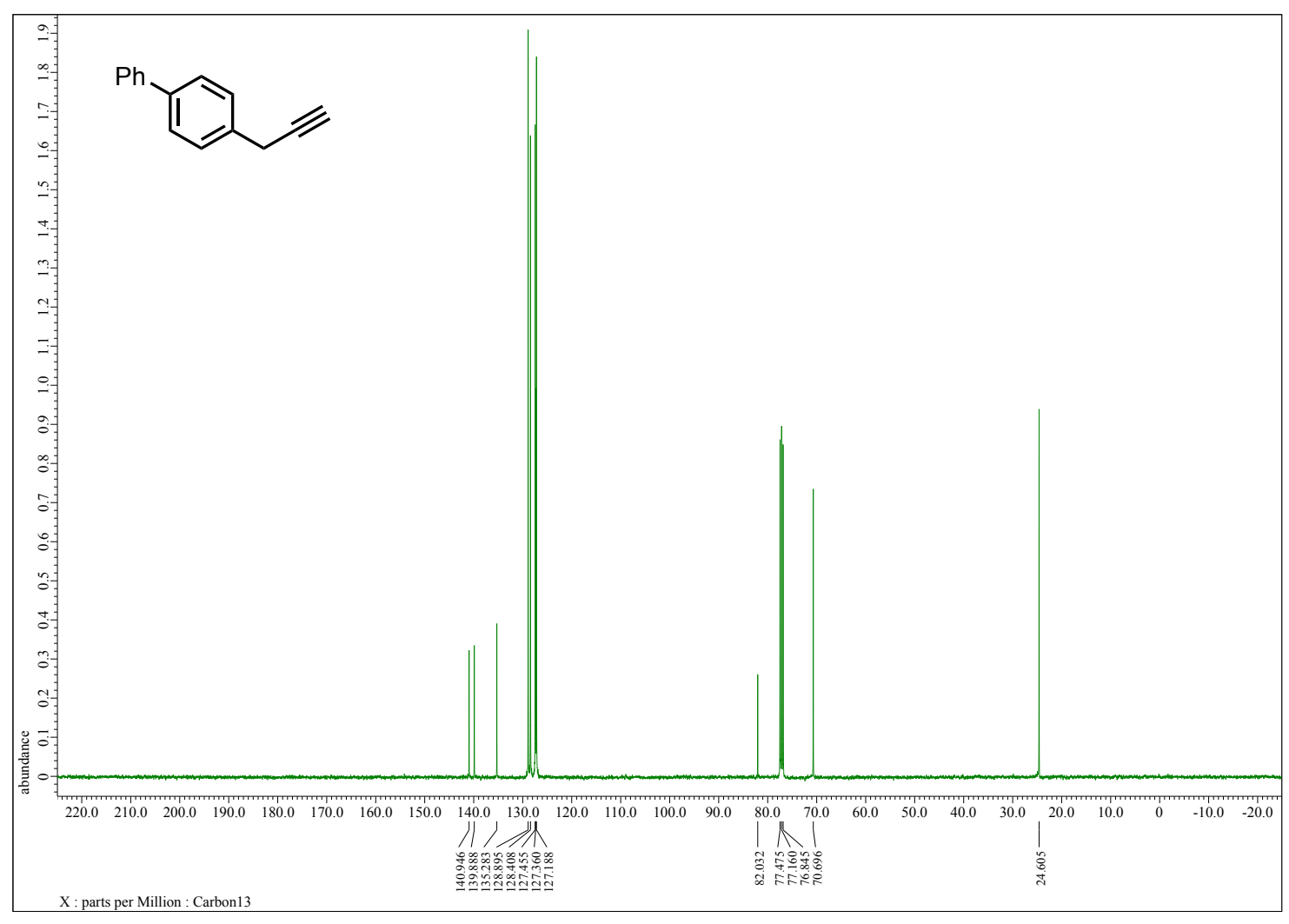

Figure S12. ${ }^{13} \mathrm{C}$ NMR of $4 a\left(\mathrm{CDCl}_{3}\right)$.

S19 


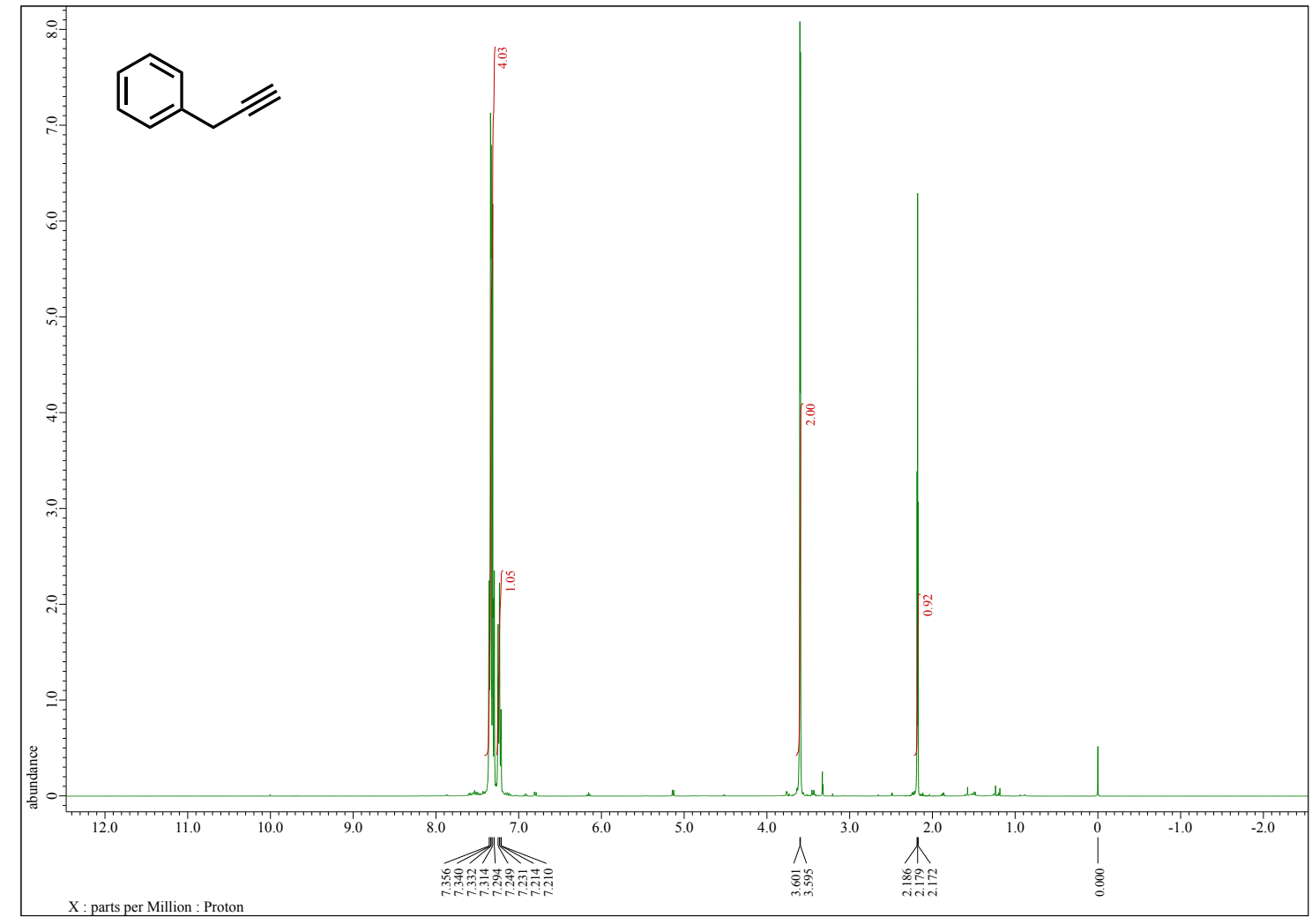

Figure S13. ${ }^{1} \mathrm{H}$ NMR of $\mathbf{4 b}\left(\mathrm{CDCl}_{3}\right)$.

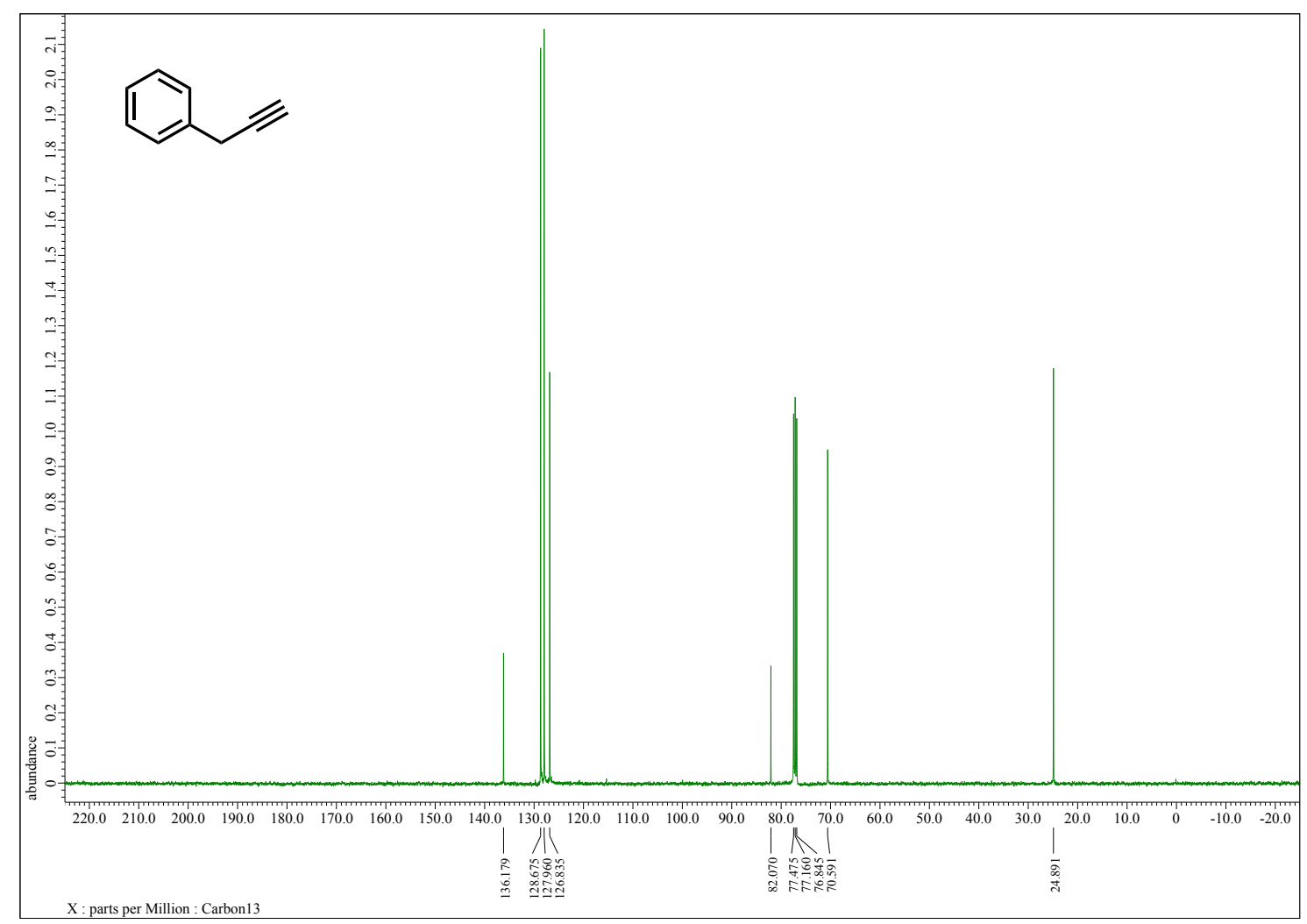

Figure S14. ${ }^{13} \mathrm{C}$ NMR of $4 \mathbf{b}\left(\mathrm{CDCl}_{3}\right)$. 


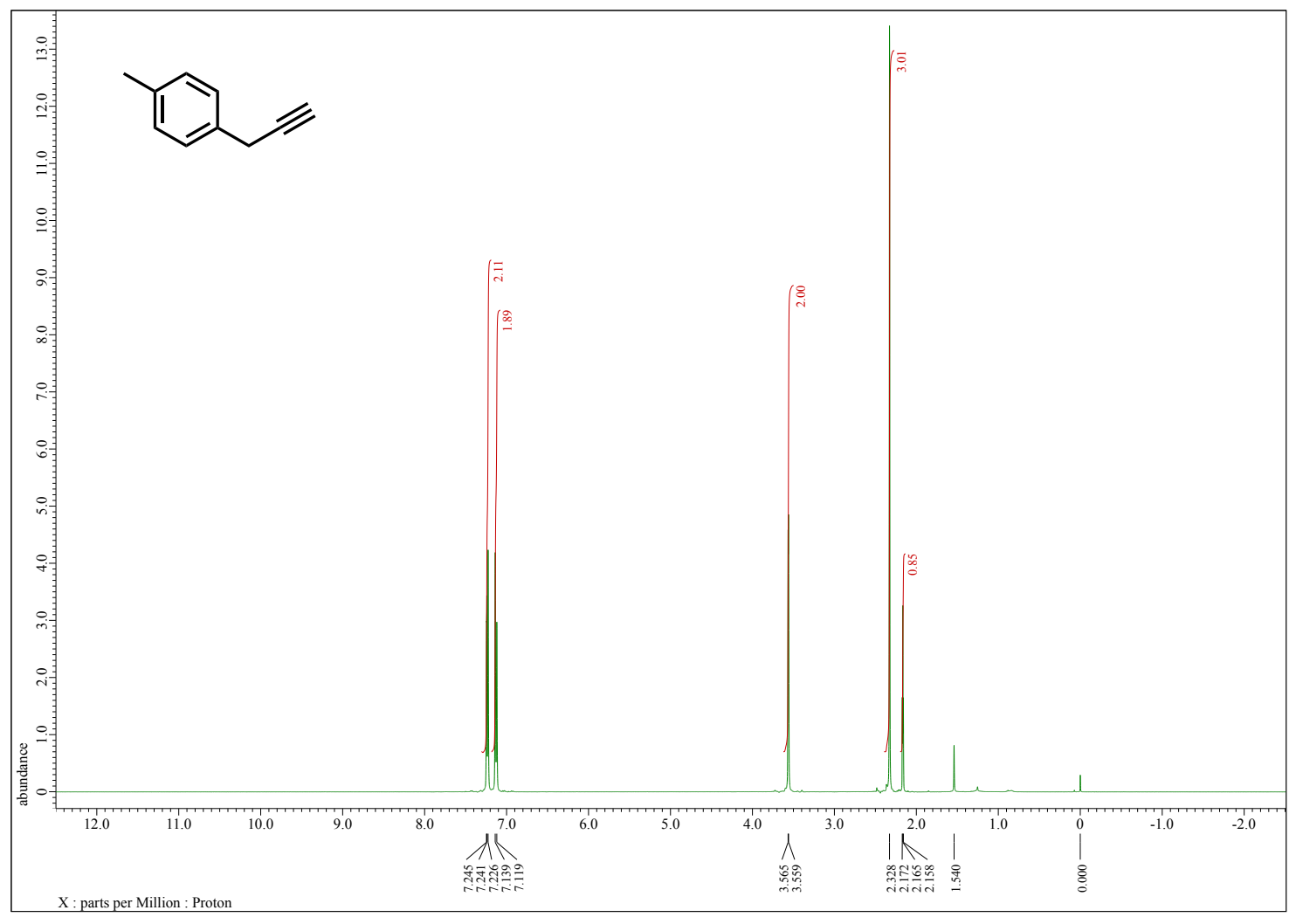

Figure S15. ${ }^{1} \mathrm{H}$ NMR of $4 \mathbf{c}\left(\mathrm{CDCl}_{3}\right)$.

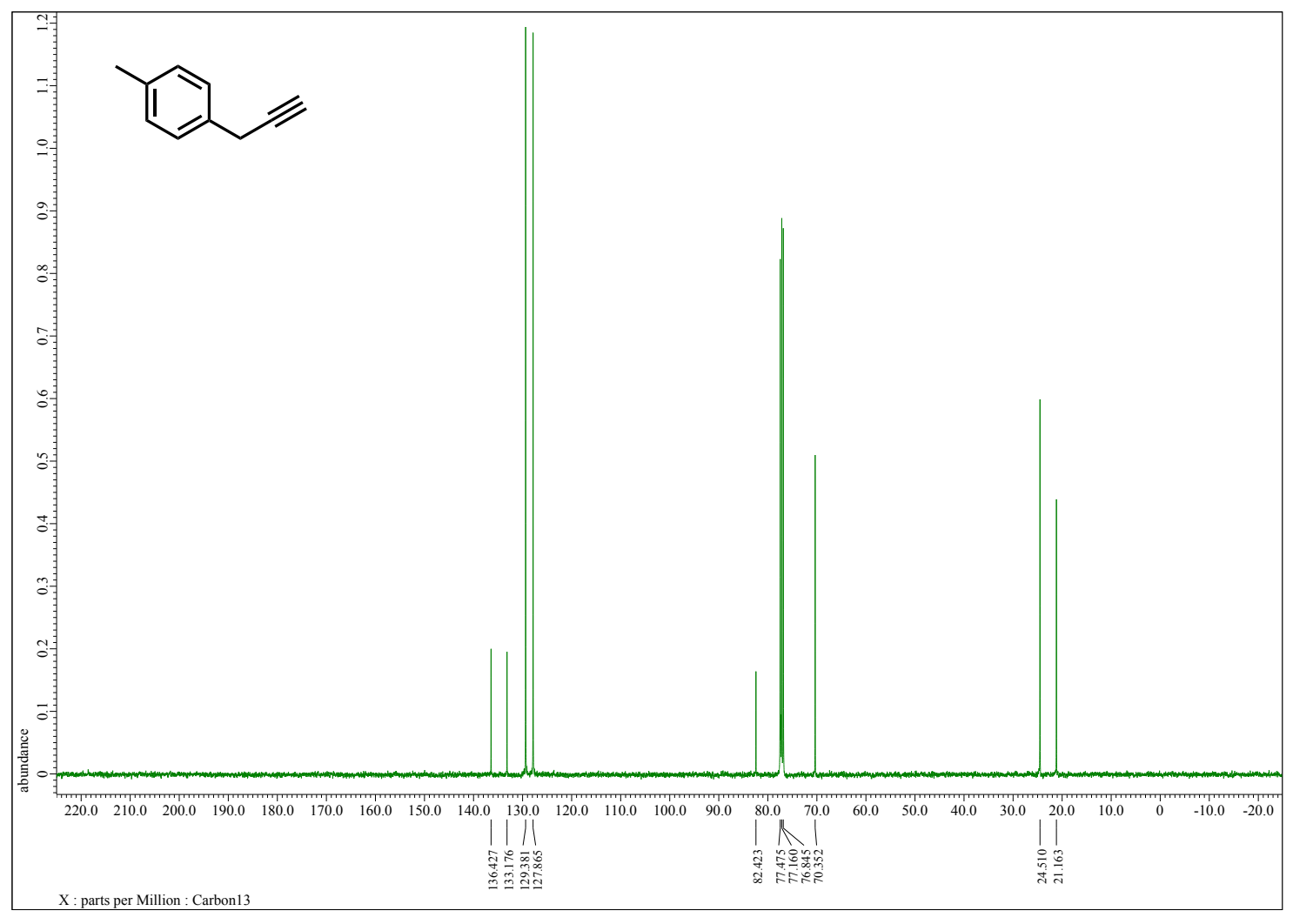

Figure S16. ${ }^{13} \mathrm{C}$ NMR of $4 \mathbf{c}\left(\mathrm{CDCl}_{3}\right)$.

S21 


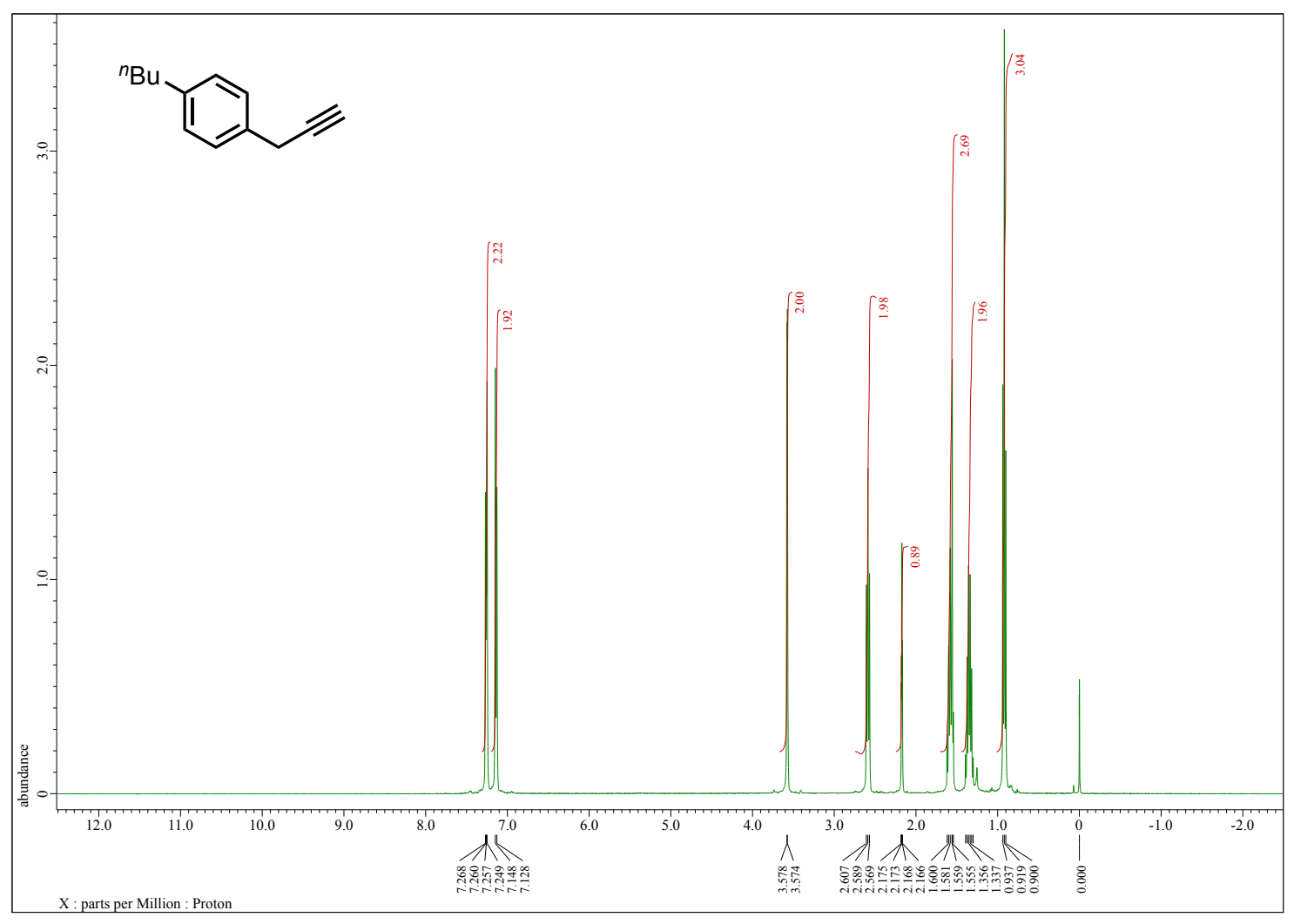

Figure S17. ${ }^{1} \mathrm{H} \mathrm{NMR}$ of $\mathbf{4 d}\left(\mathrm{CDCl}_{3}\right)$.

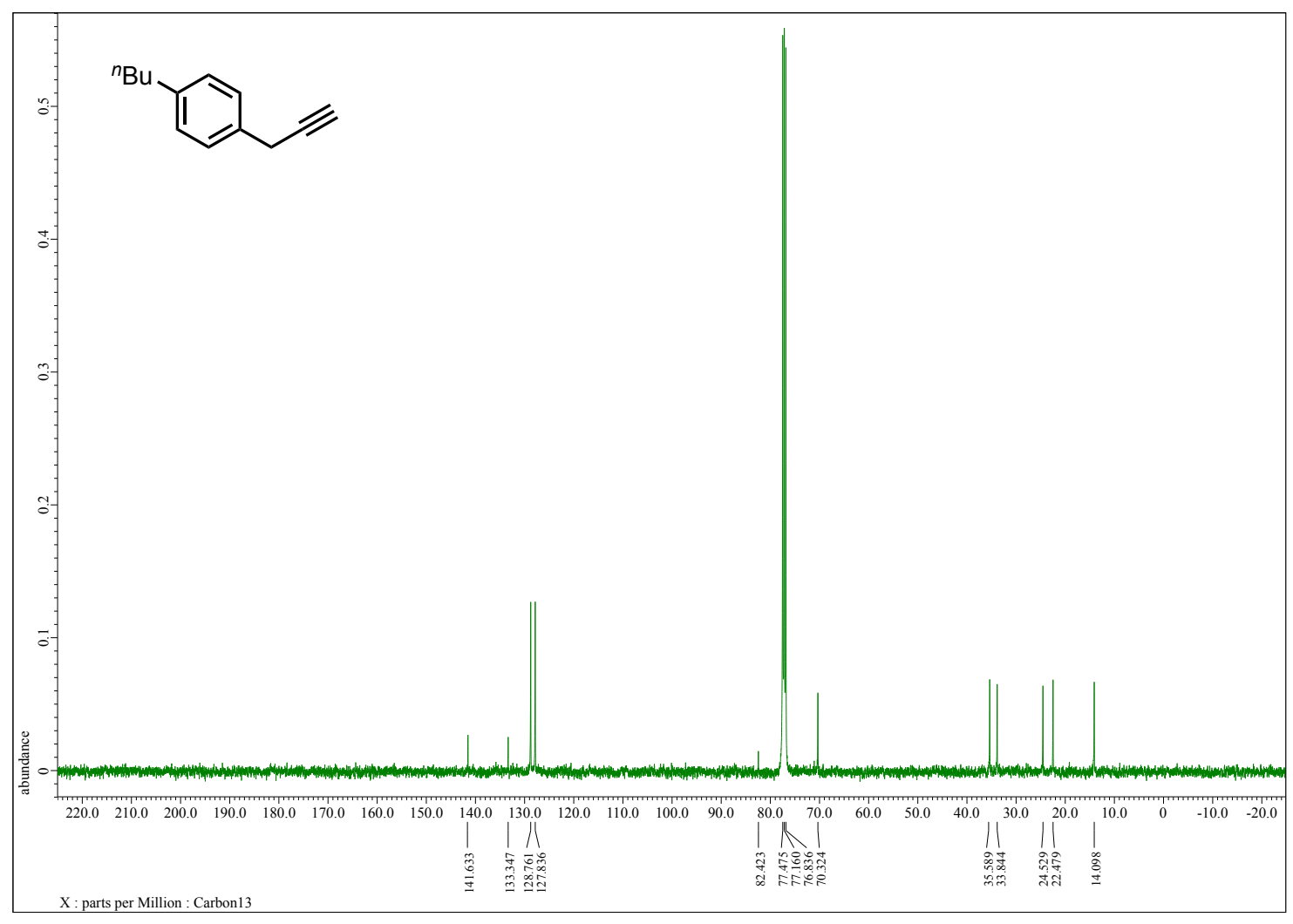

Figure S18. ${ }^{13} \mathrm{C}$ NMR of $4 d\left(\mathrm{CDCl}_{3}\right)$.

S22 


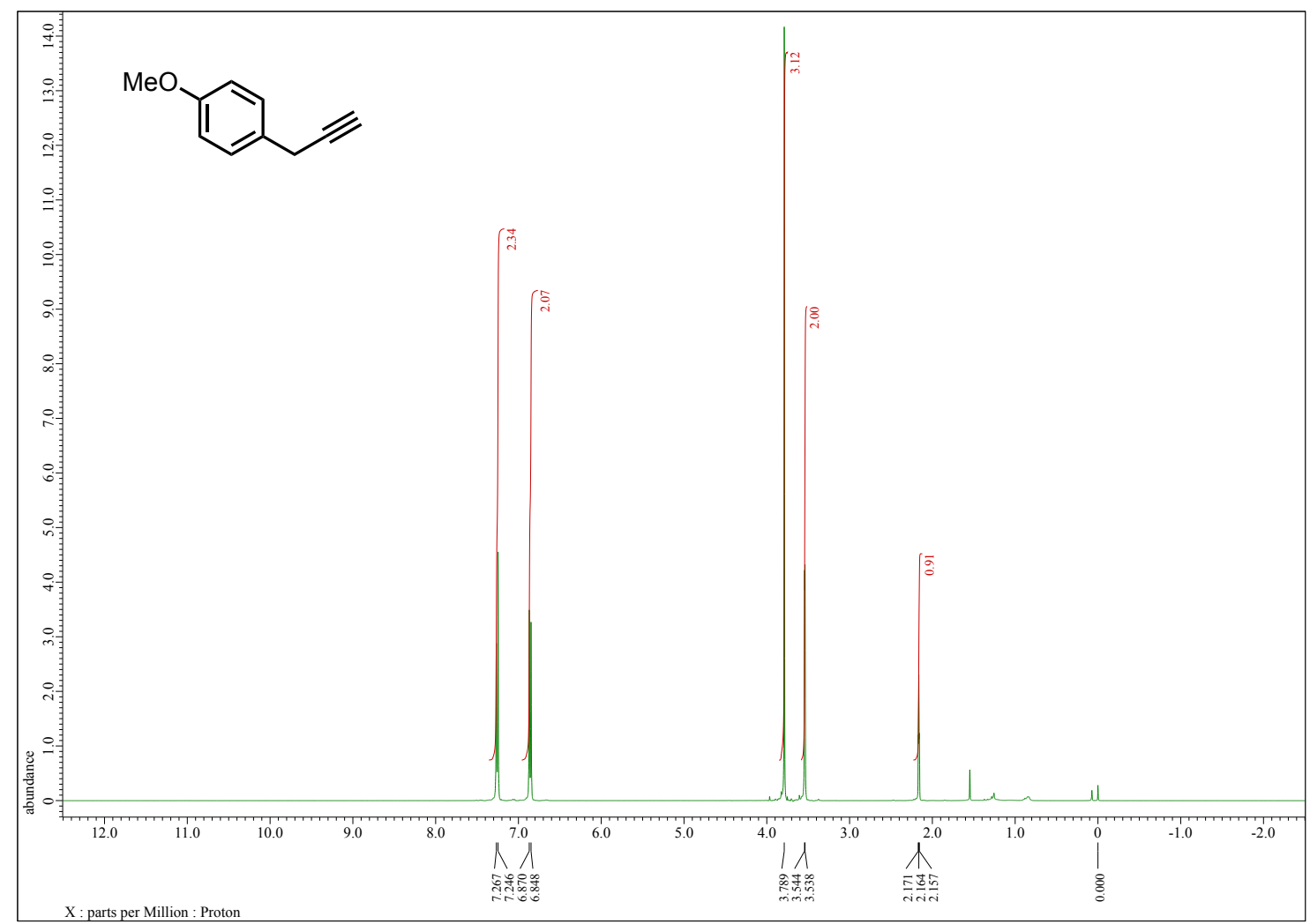

Figure S19. ${ }^{1} \mathrm{H}$ NMR of $4 \mathbf{e}\left(\mathrm{CDCl}_{3}\right)$.

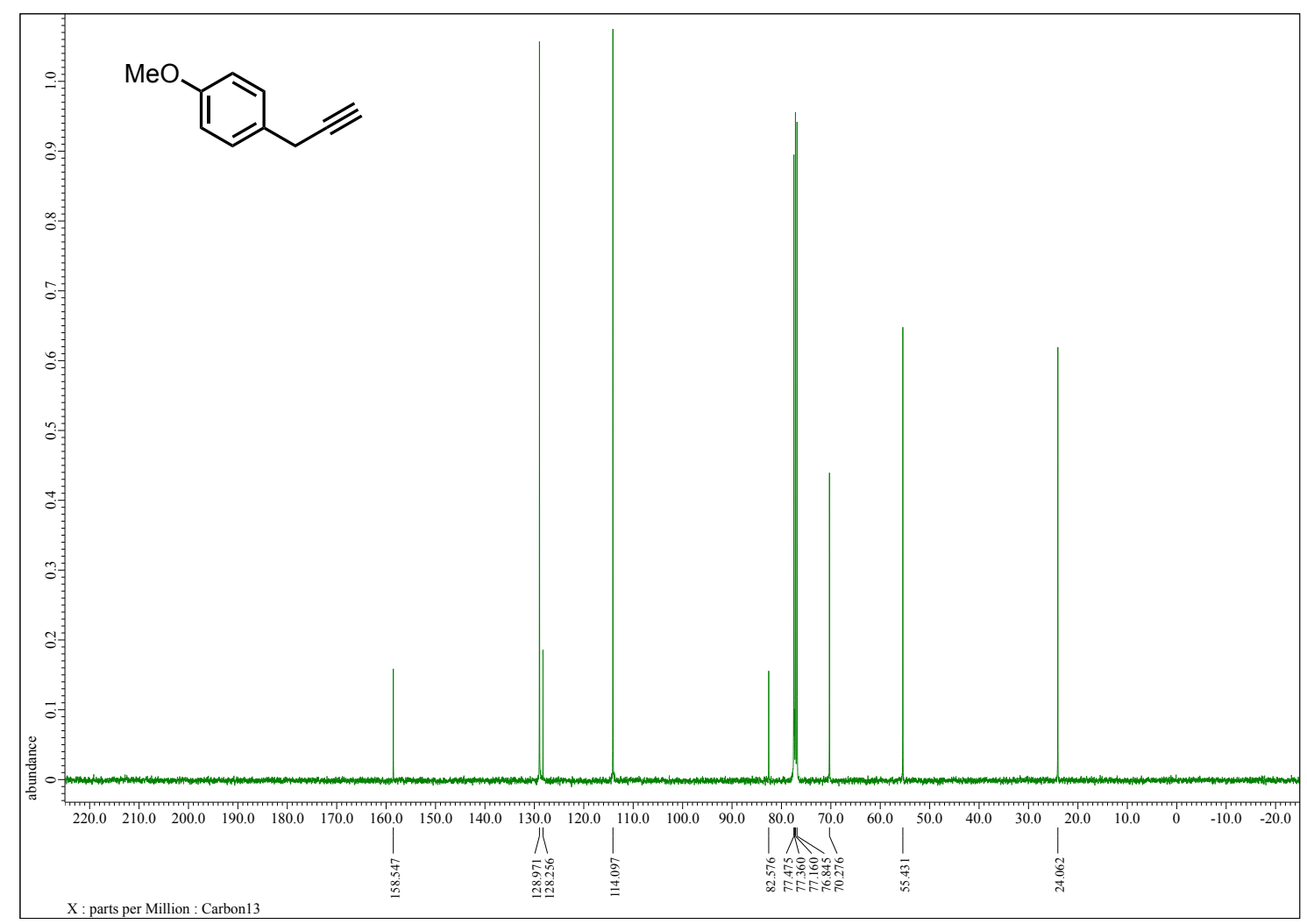

Figure S20. ${ }^{13} \mathrm{C}$ NMR of $4 \mathrm{e}\left(\mathrm{CDCl}_{3}\right)$. 


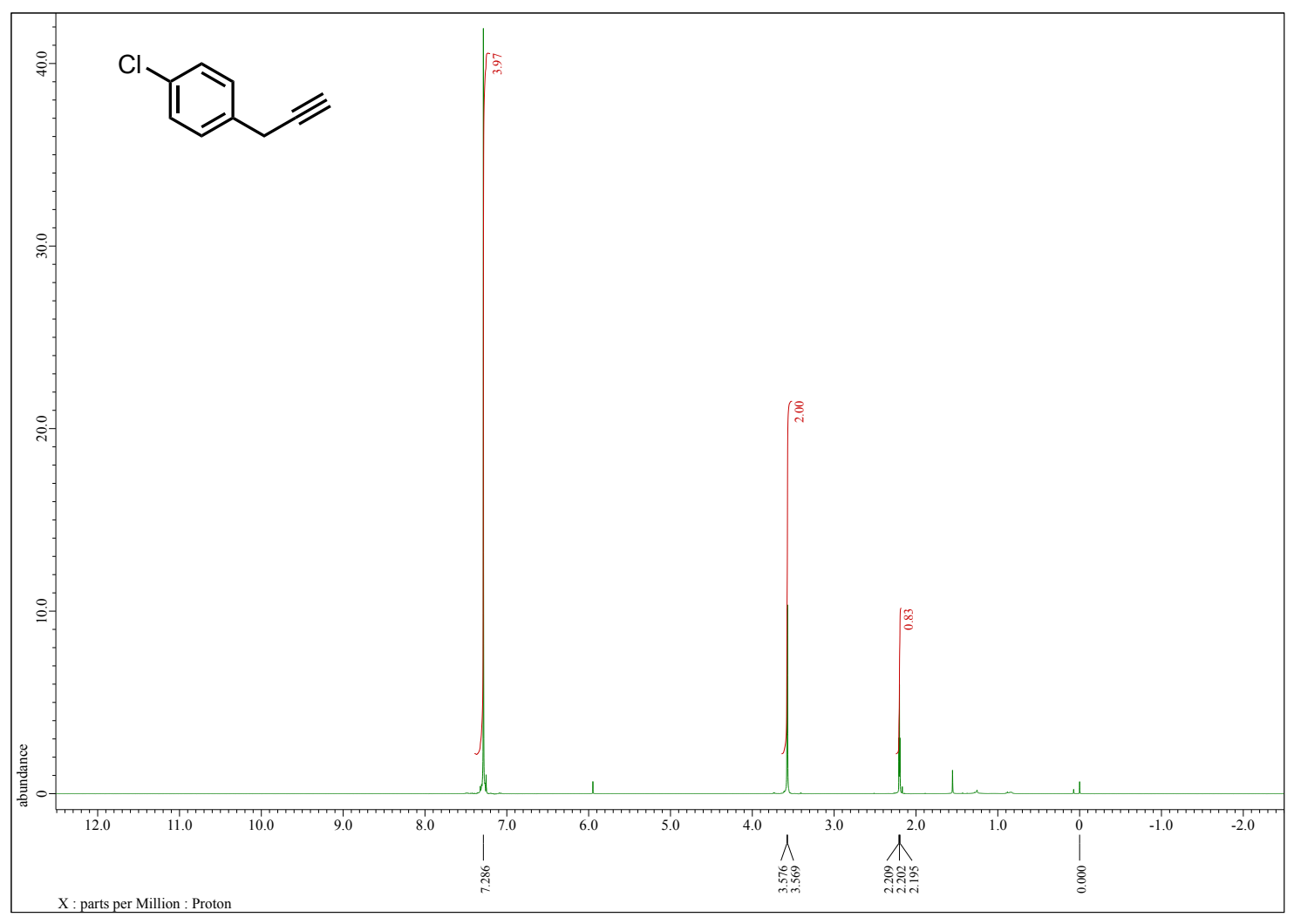

Figure S21. ${ }^{1} \mathrm{H}$ NMR of $\mathbf{4 f}\left(\mathrm{CDCl}_{3}\right)$.

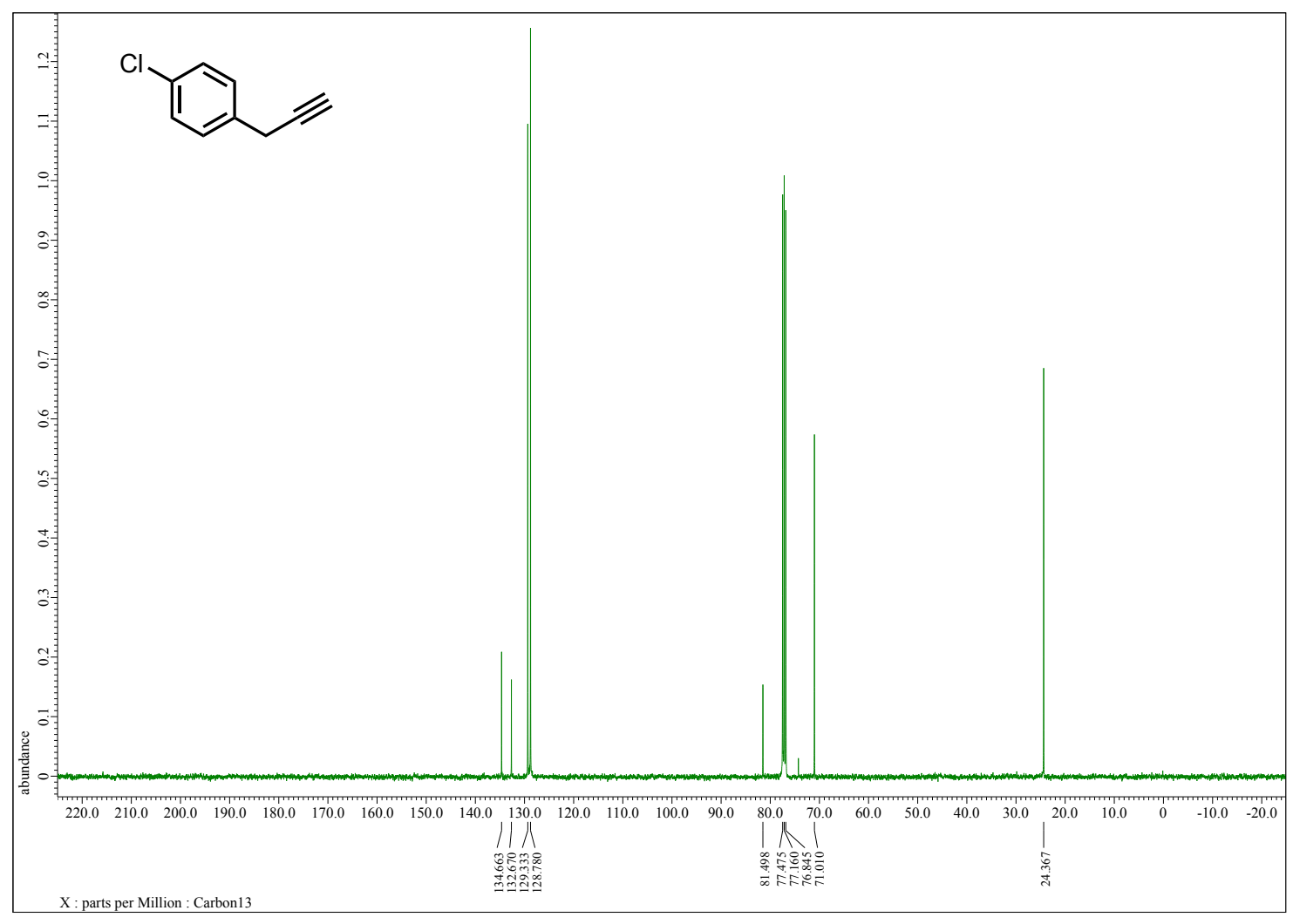

Figure S22. ${ }^{13} \mathrm{C}$ NMR of $\mathbf{4 f}\left(\mathrm{CDCl}_{3}\right)$.

S24 


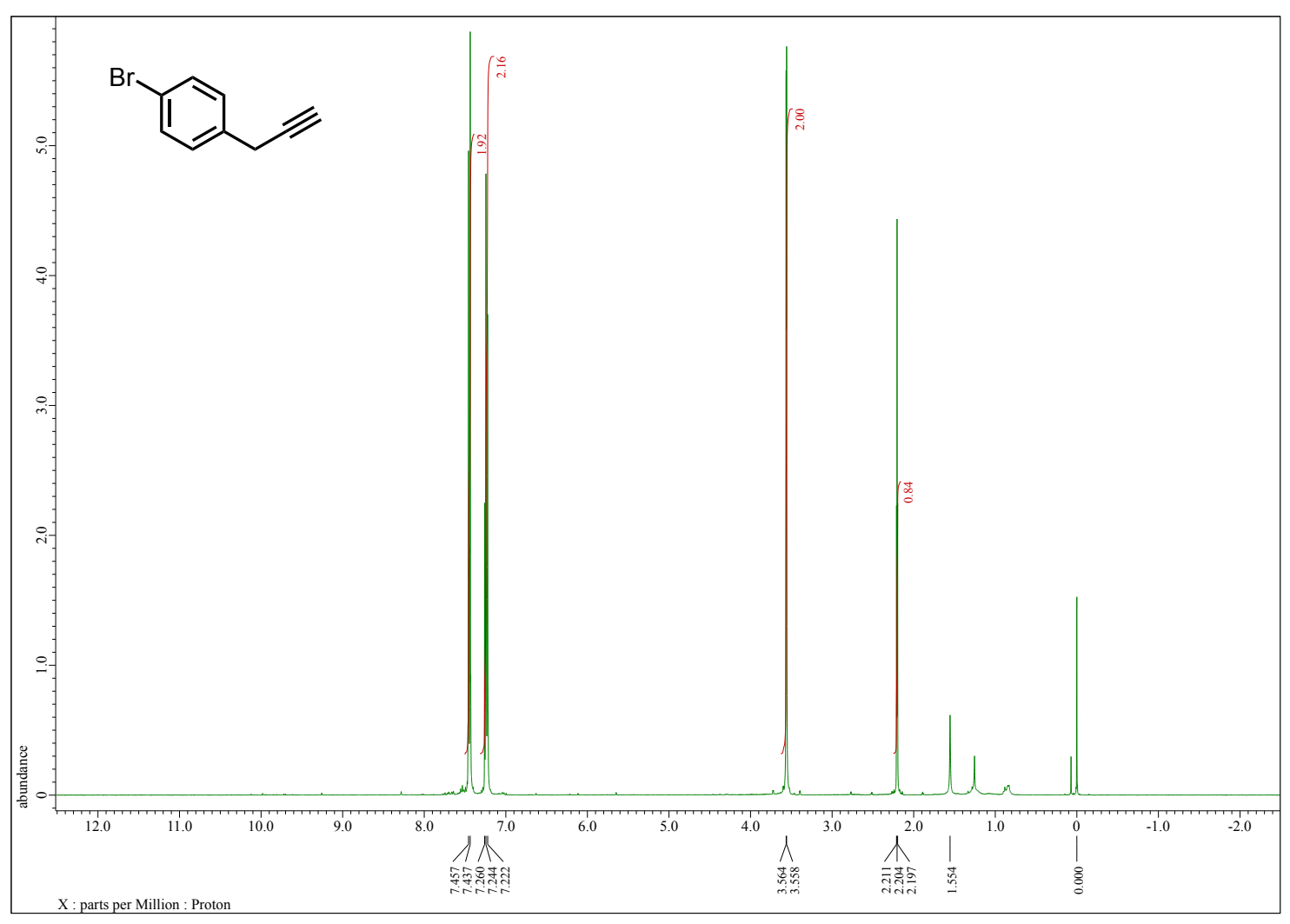

Figure S23. ${ }^{1} \mathrm{H} \mathrm{NMR}$ of $\mathbf{4 g}\left(\mathrm{CDCl}_{3}\right)$.

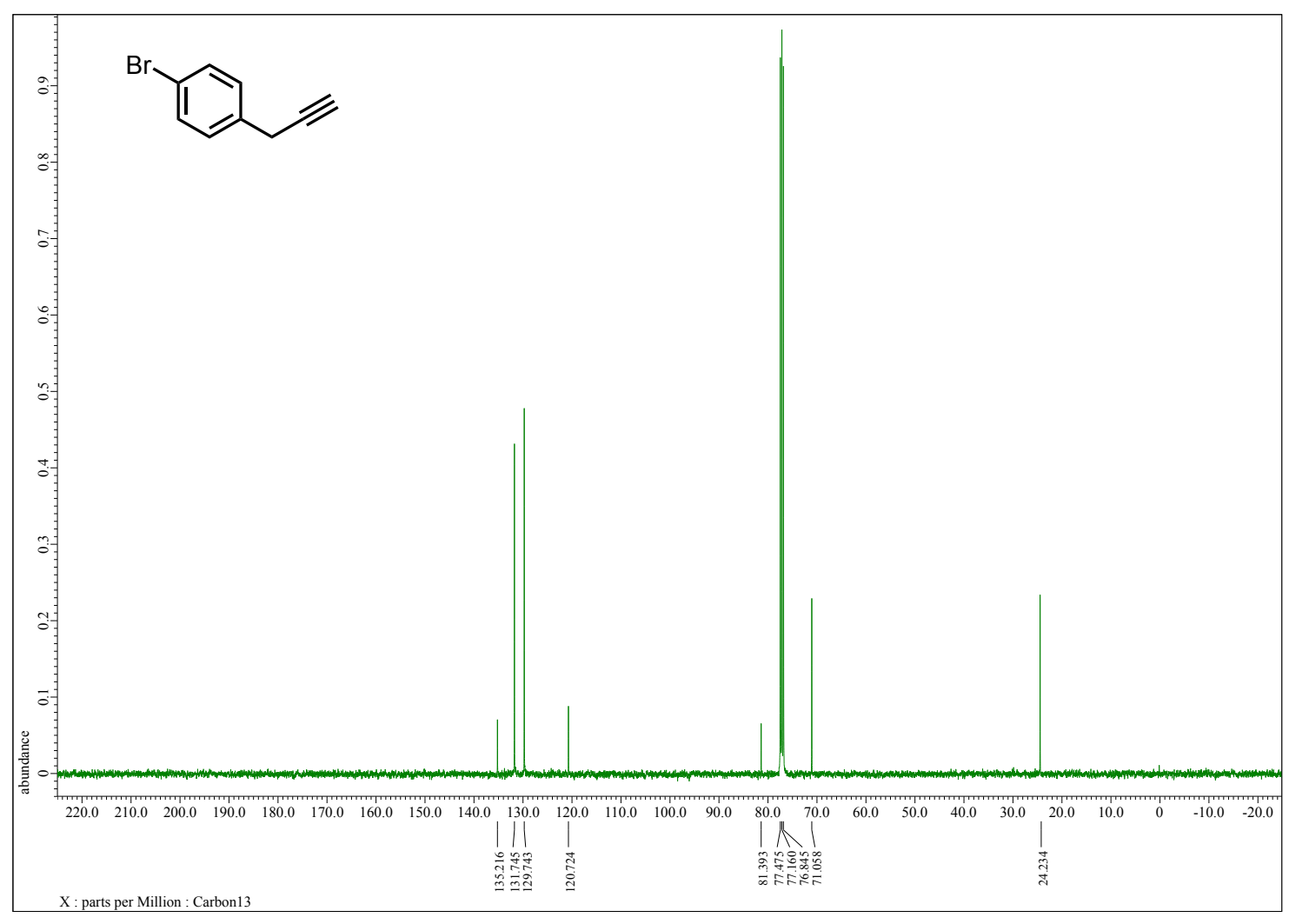

Figure S24. ${ }^{13} \mathrm{C}$ NMR of $4 \mathbf{g}\left(\mathrm{CDCl}_{3}\right)$.

S25 


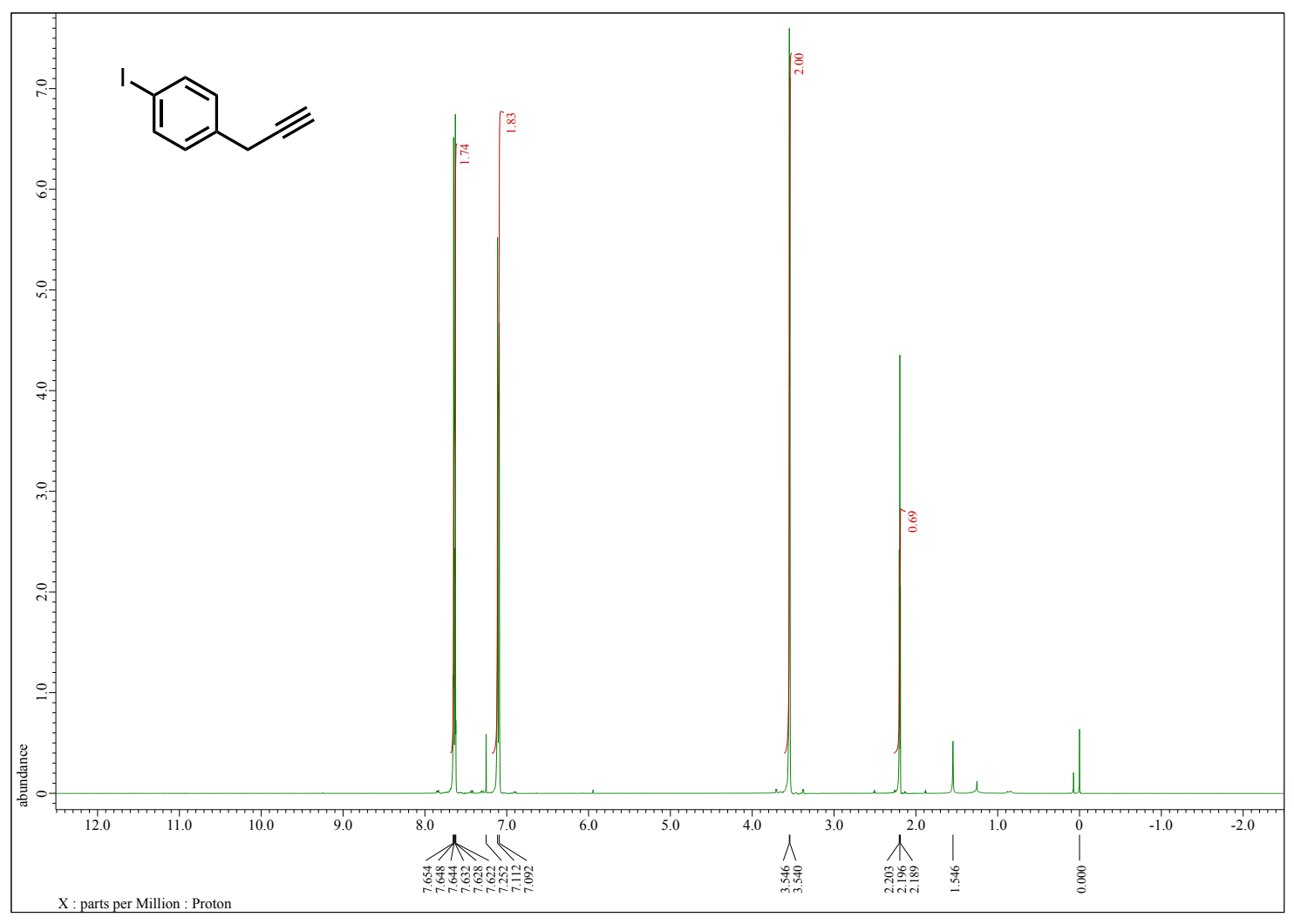

Figure S25. ${ }^{1} \mathrm{H}$ NMR of $\mathbf{4 h}\left(\mathrm{CDCl}_{3}\right)$.

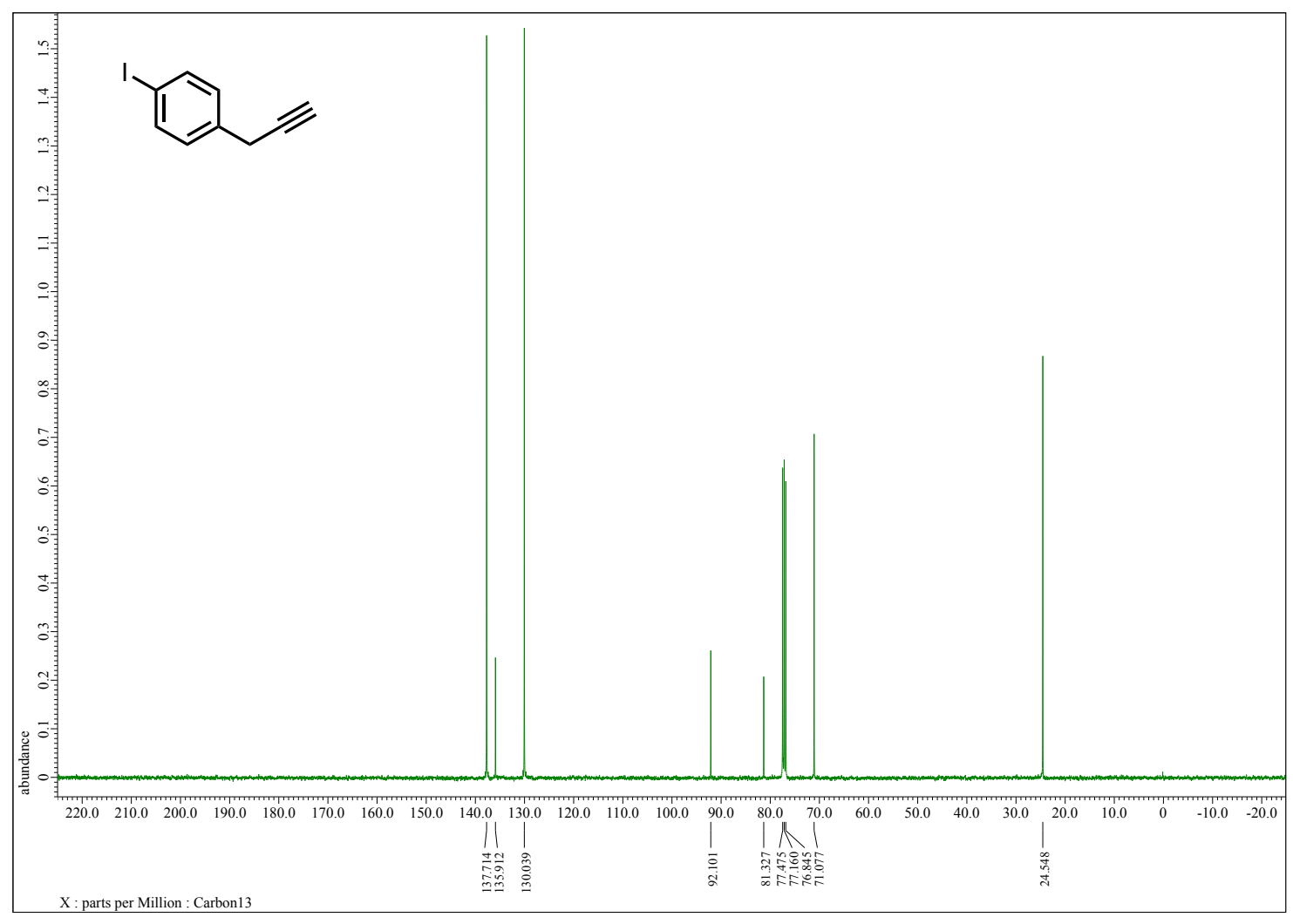

Figure S26. ${ }^{13} \mathrm{C}$ NMR of $\mathbf{4 h}\left(\mathrm{CDCl}_{3}\right)$.

S26 


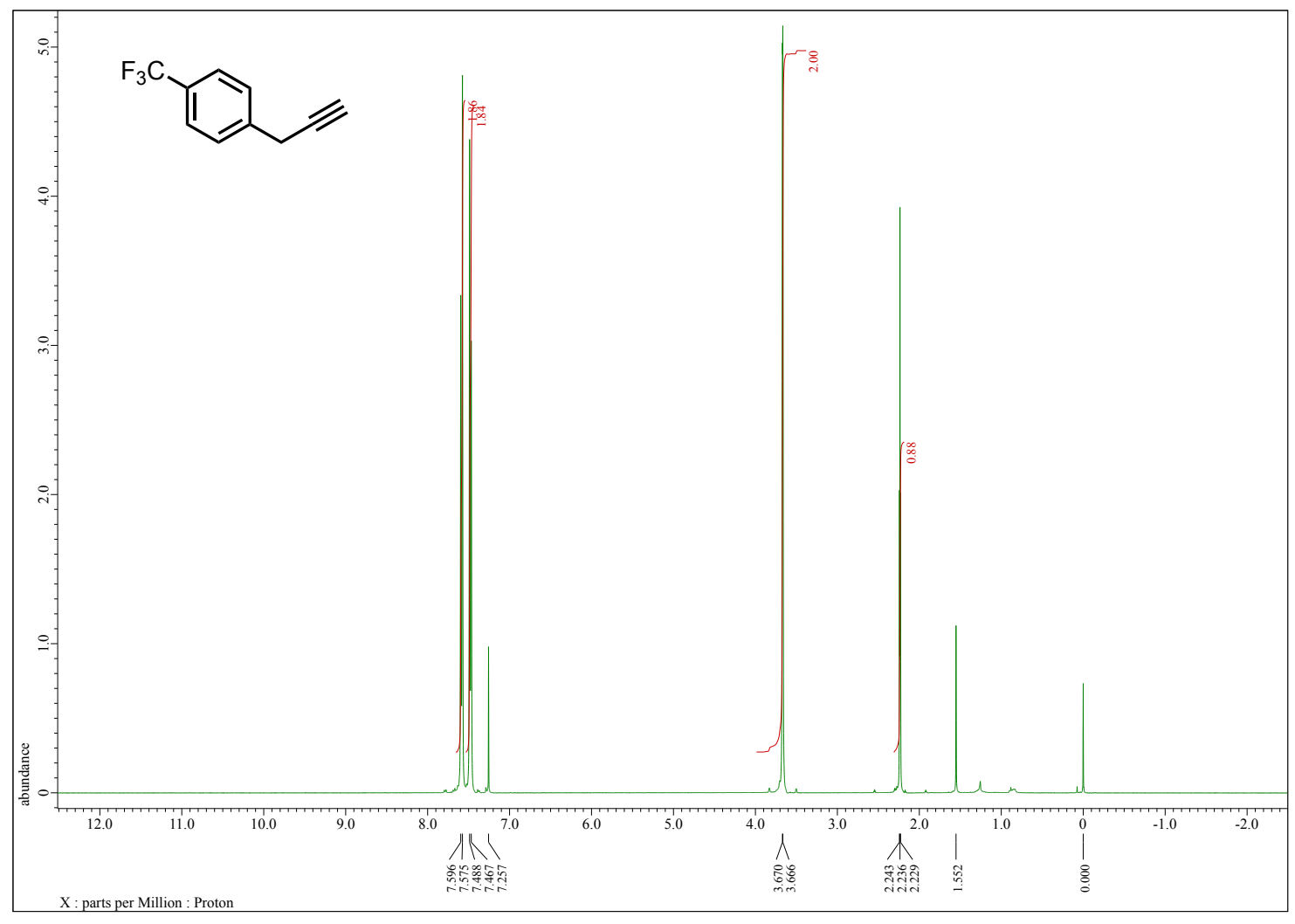

Figure S27. ${ }^{1} \mathrm{H}$ NMR of $4 \mathbf{i}\left(\mathrm{CDCl}_{3}\right)$.

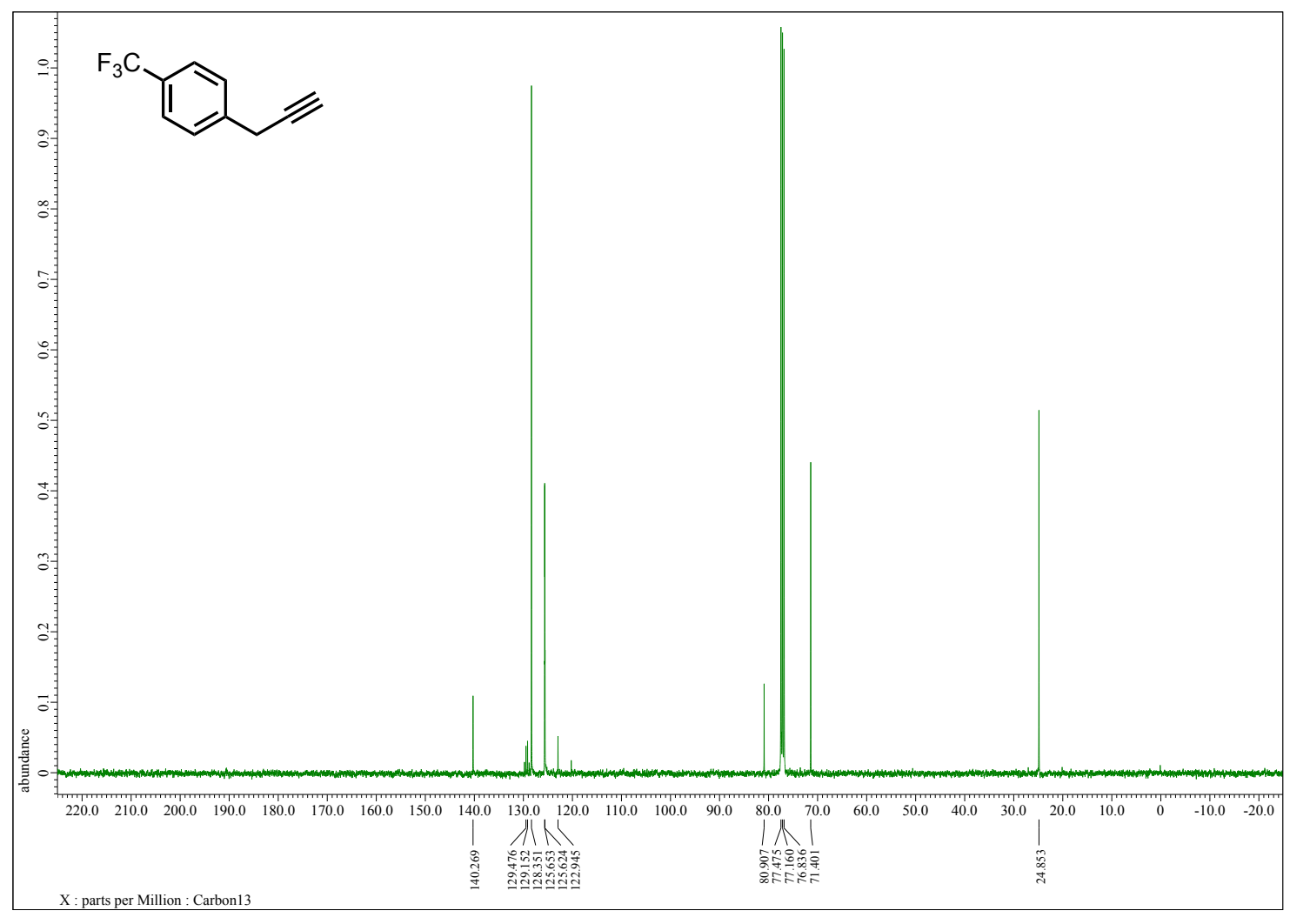

Figure S28. ${ }^{13} \mathrm{C}$ NMR of $4 \mathbf{i}\left(\mathrm{CDCl}_{3}\right)$.

S27 


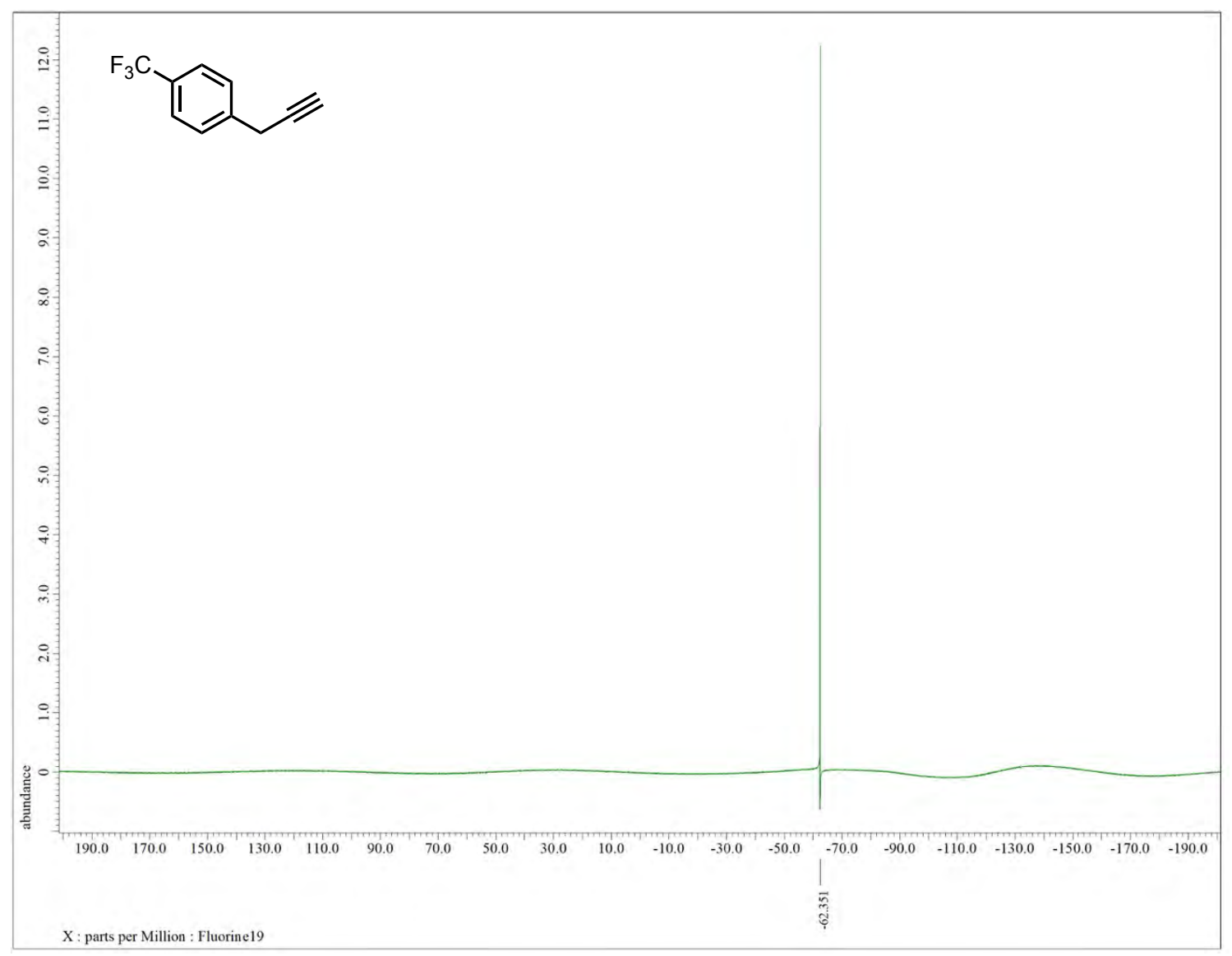

Figure S29. ${ }^{19} \mathrm{~F}$ NMR of $4 \mathbf{i}\left(\mathrm{CDCl}_{3}\right)$.

S28 


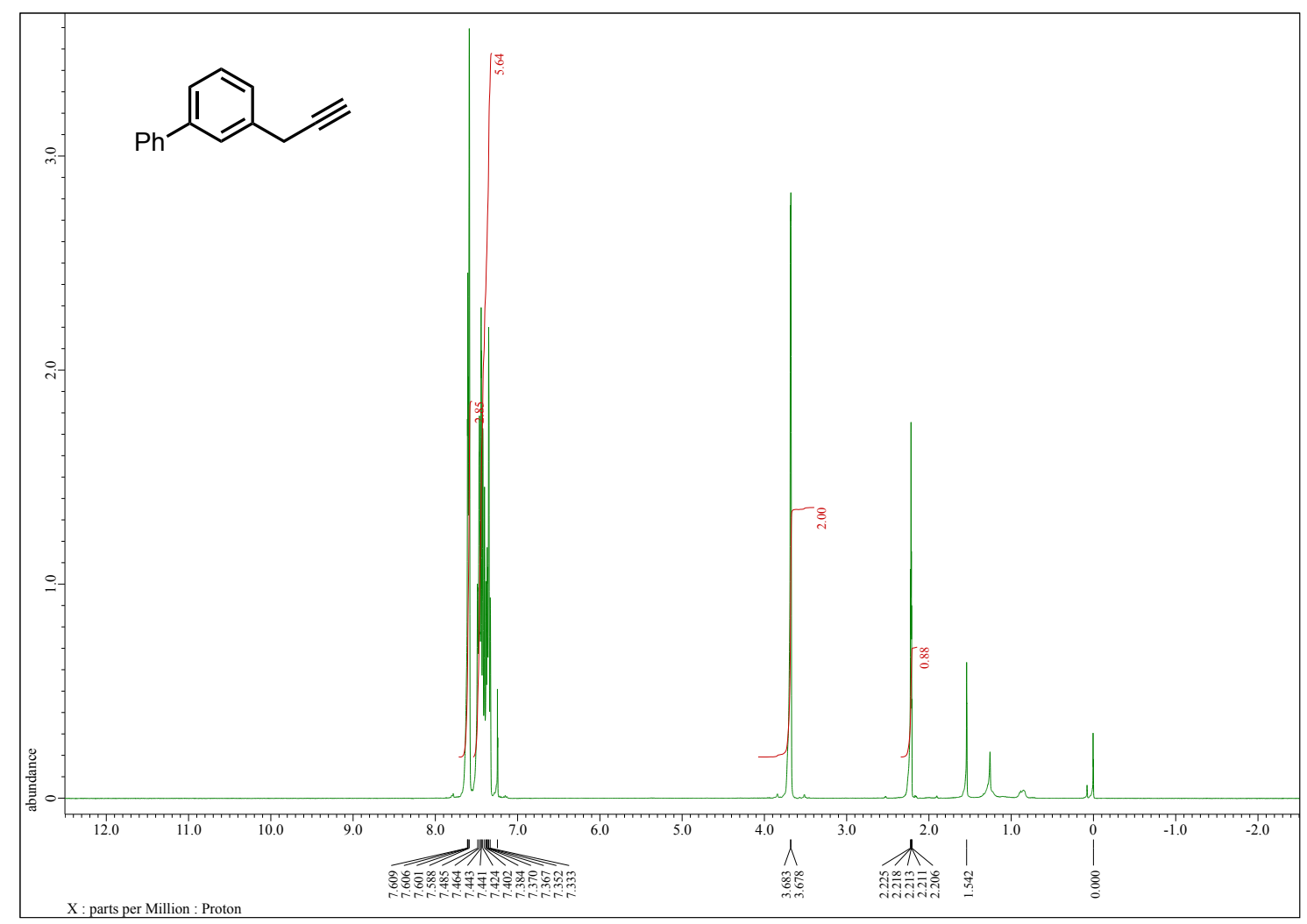

Figure S30. ${ }^{1} \mathrm{H}$ NMR of $\mathbf{4 j}\left(\mathrm{CDCl}_{3}\right)$.

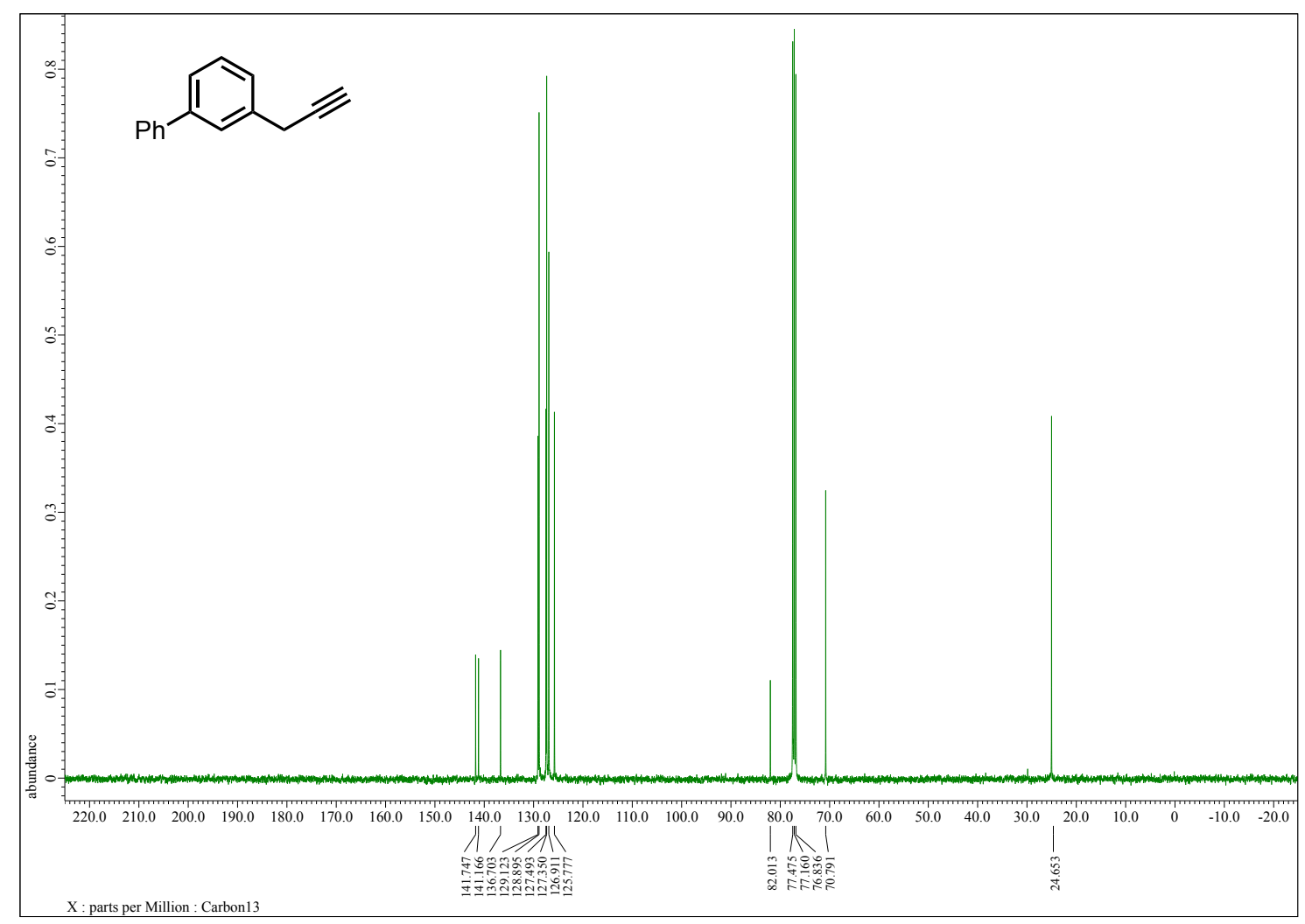

Figure S31. ${ }^{13} \mathrm{C}$ NMR of $\mathbf{4 j}\left(\mathrm{CDCl}_{3}\right)$. 


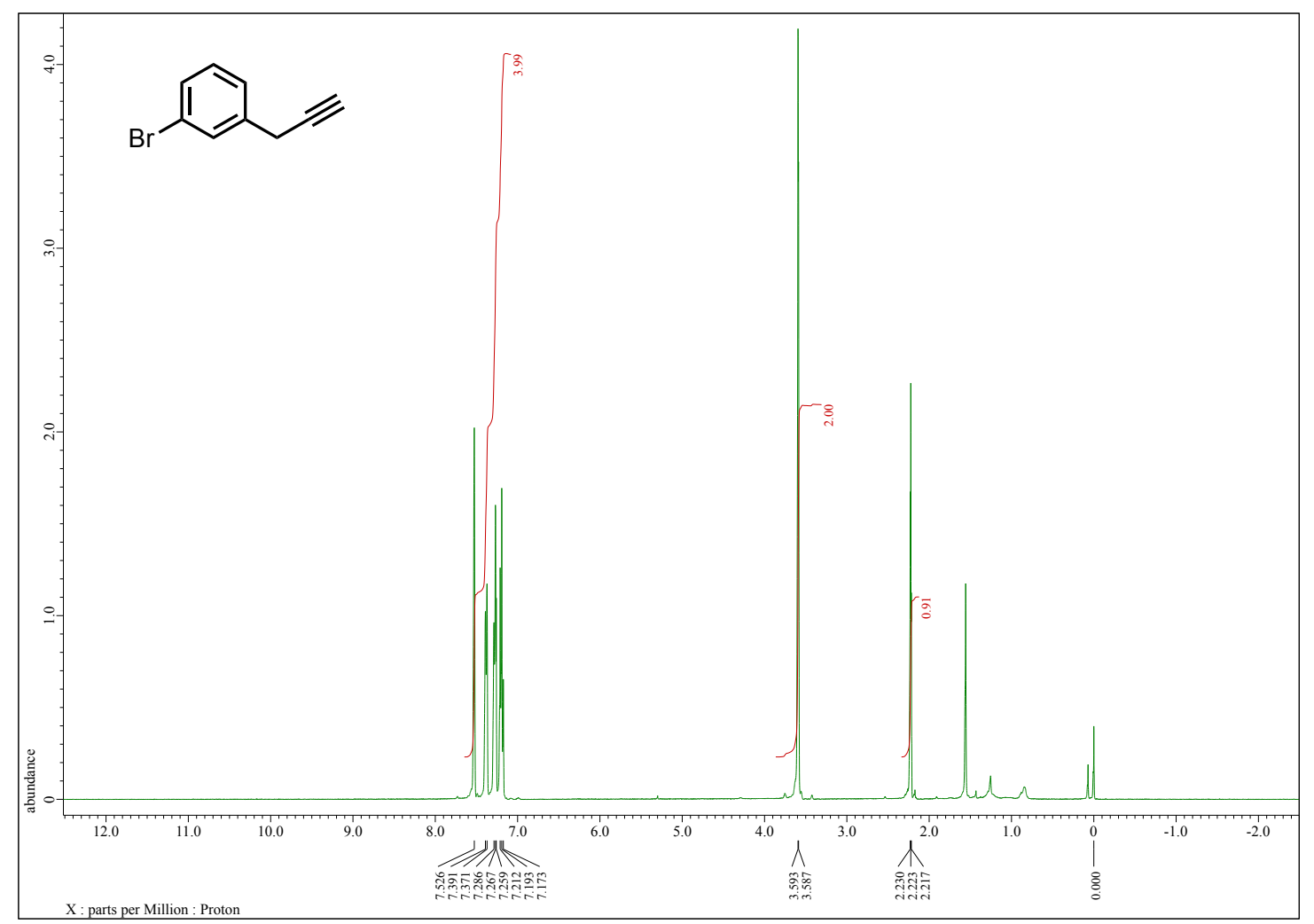

Figure S32. ${ }^{1} \mathrm{H}$ NMR of $\mathbf{4 k}\left(\mathrm{CDCl}_{3}\right)$.

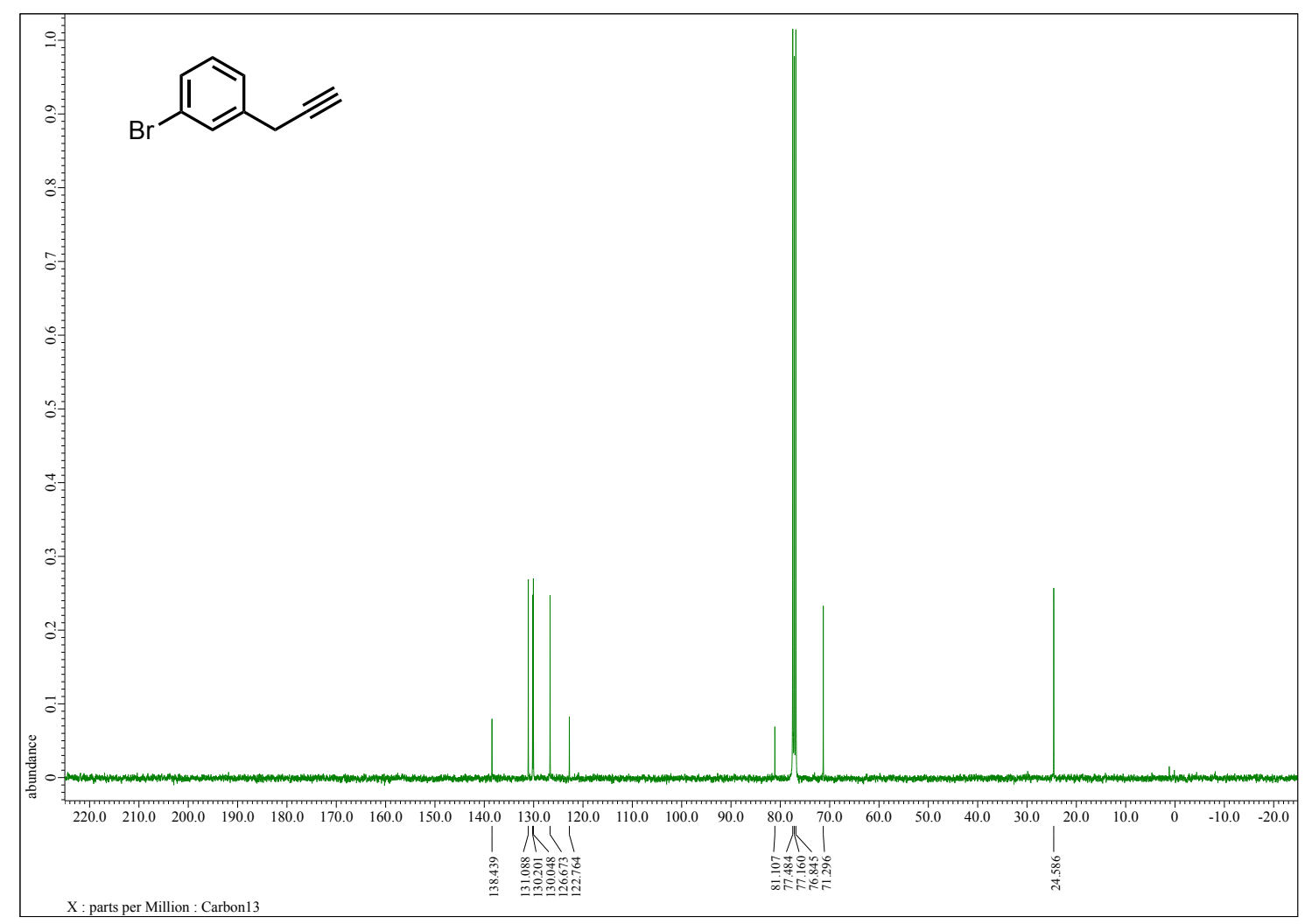

Figure S33. ${ }^{13} \mathrm{C}$ NMR of $4 \mathbf{k}\left(\mathrm{CDCl}_{3}\right)$. 


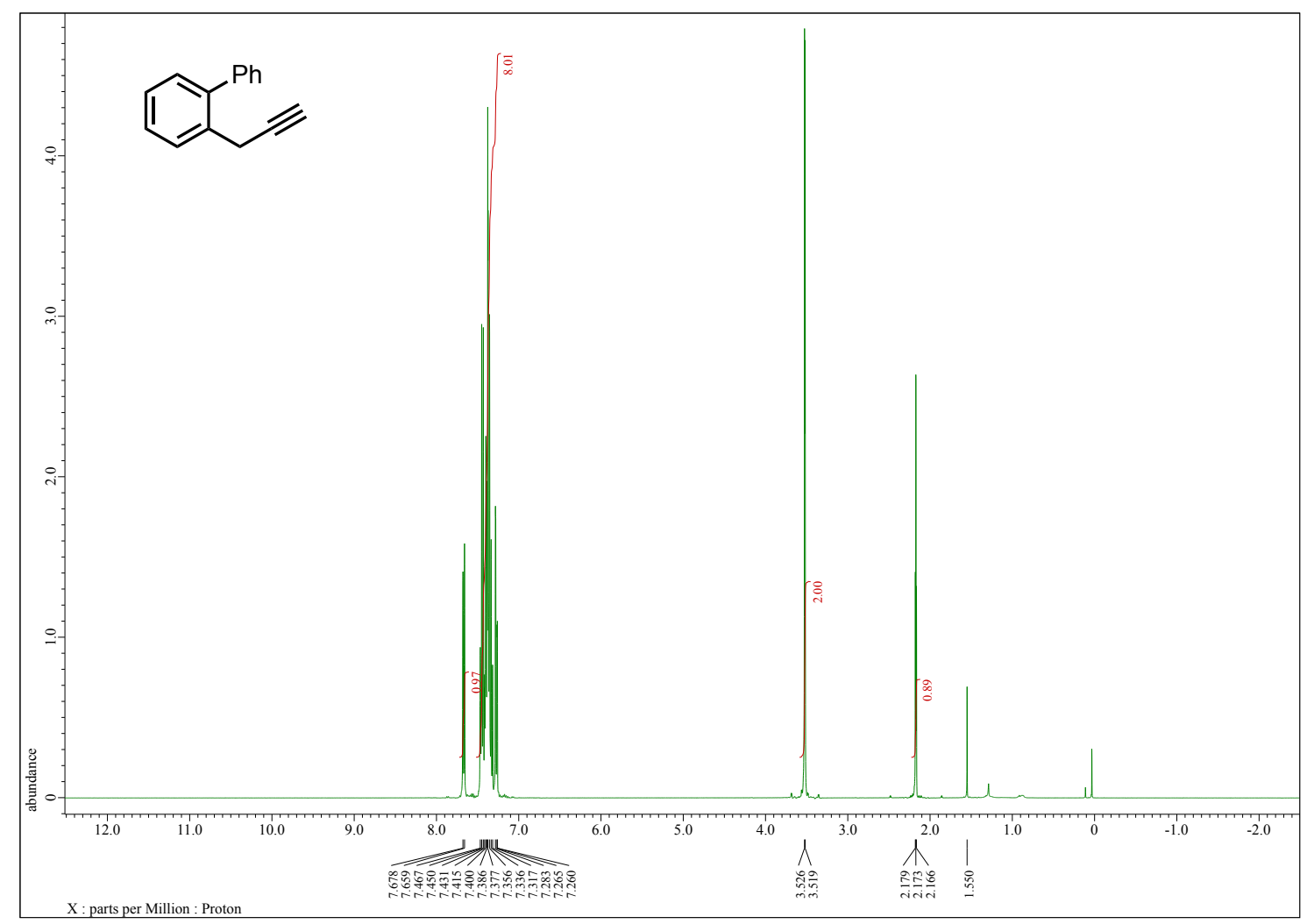

Figure S34. ${ }^{1} \mathrm{H} \mathrm{NMR}$ of $4 \mathrm{l}\left(\mathrm{CDCl}_{3}\right)$.

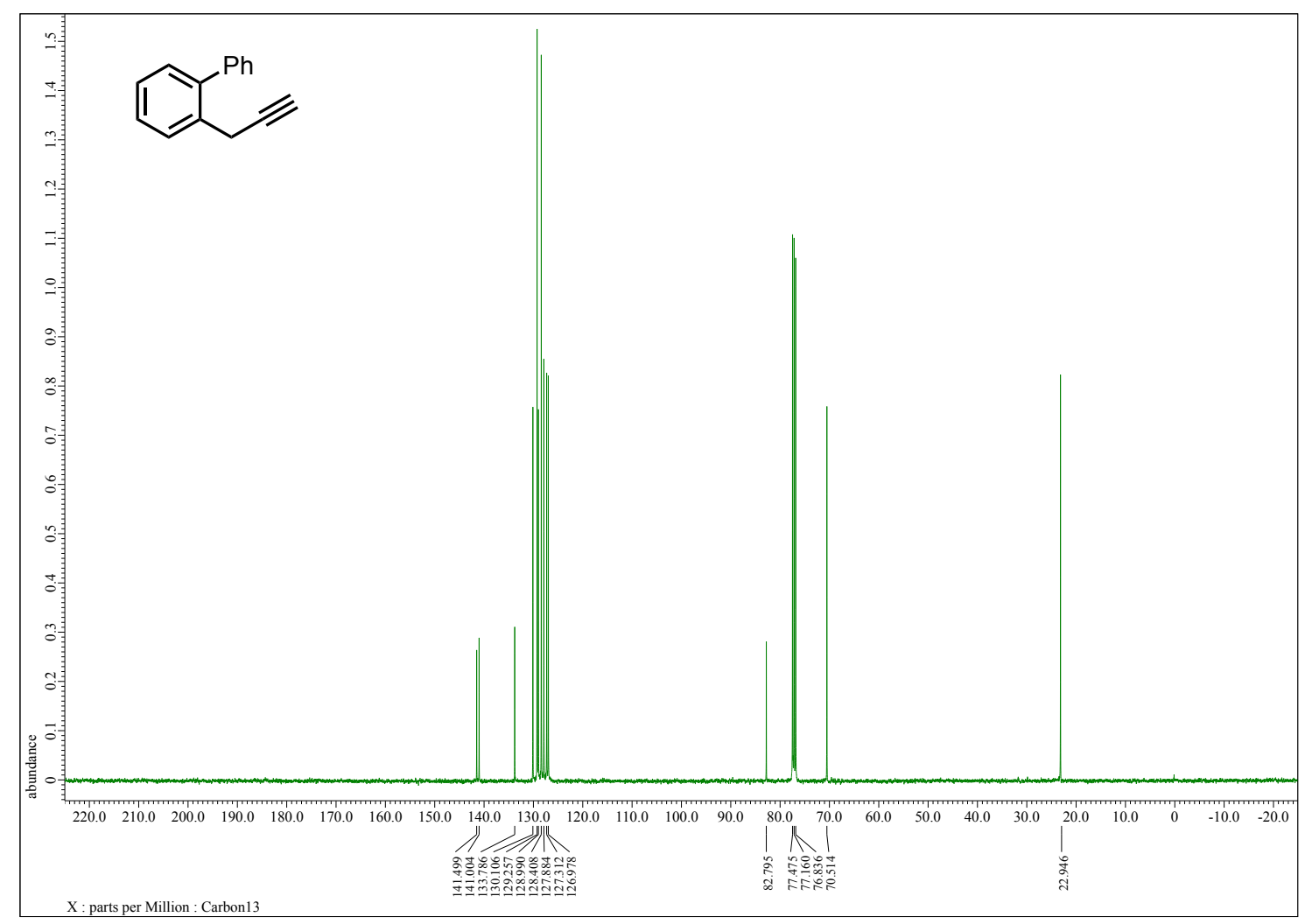

Figure S35. ${ }^{13} \mathrm{C}$ NMR of $4 \mathrm{l}\left(\mathrm{CDCl}_{3}\right)$. 


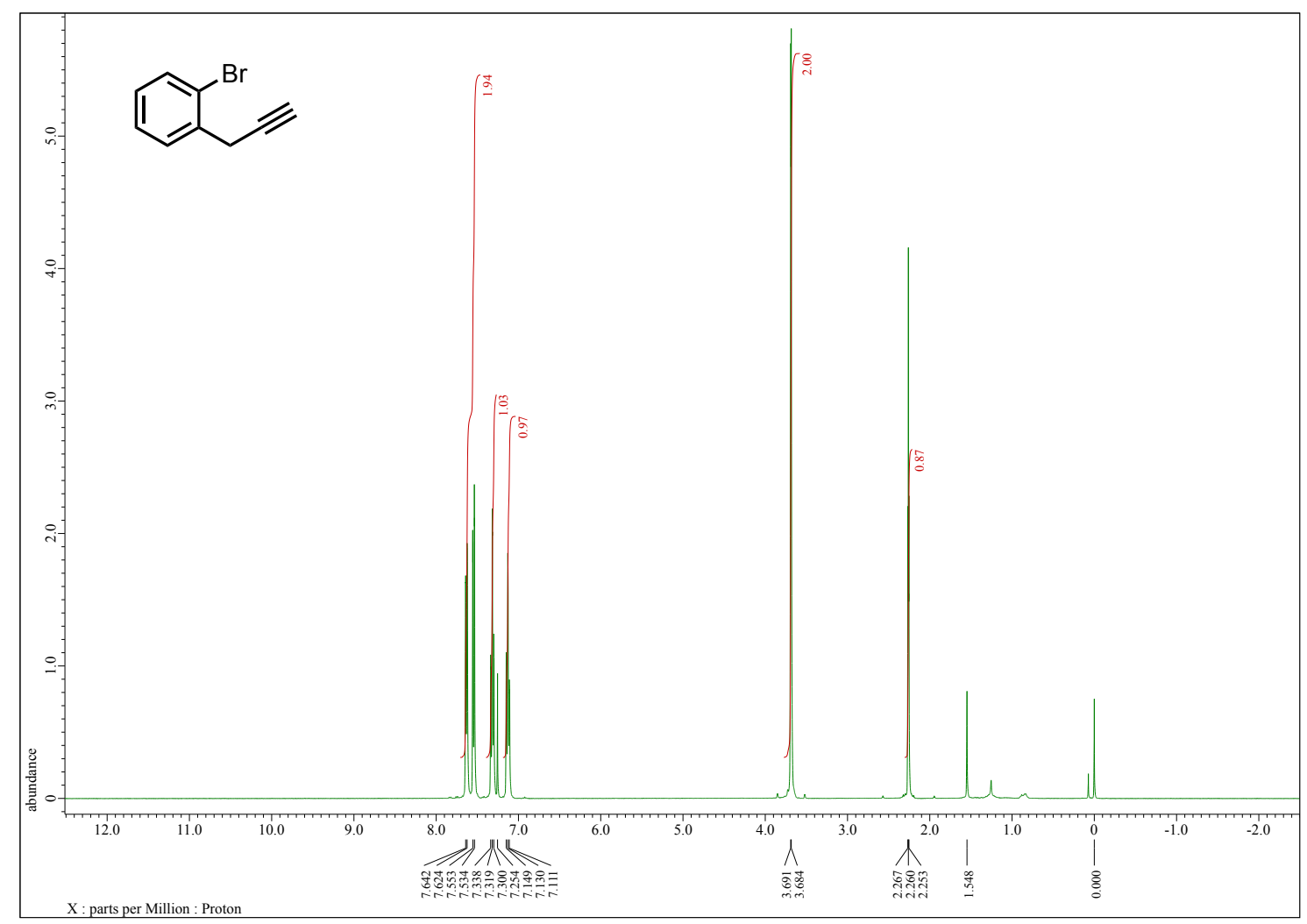

Figure S36. ${ }^{1} \mathrm{H}$ NMR of $\mathbf{4 m}\left(\mathrm{CDCl}_{3}\right)$.

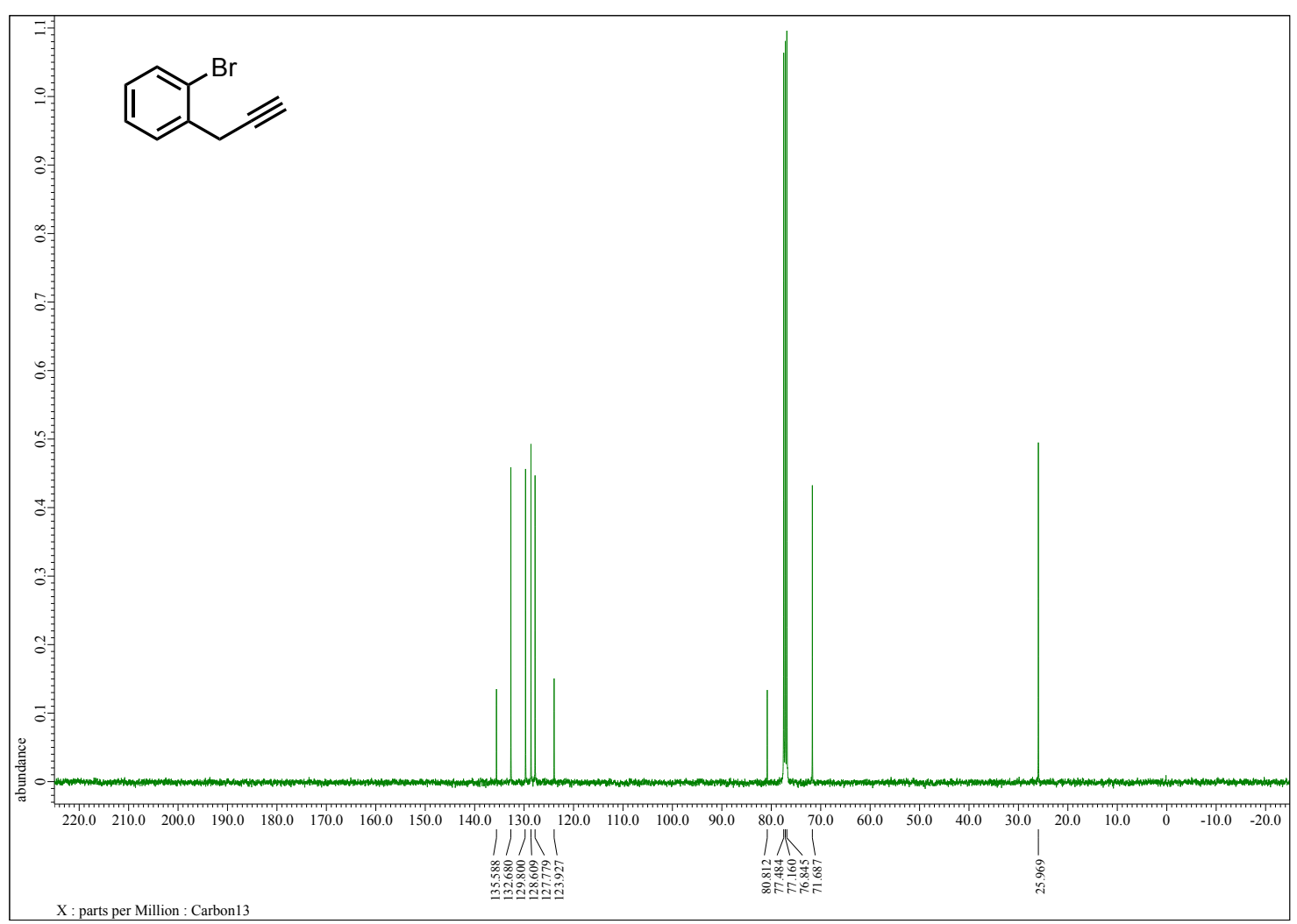

Figure S37. ${ }^{13} \mathrm{C}$ NMR of $4 \mathbf{m}\left(\mathrm{CDCl}_{3}\right)$. 


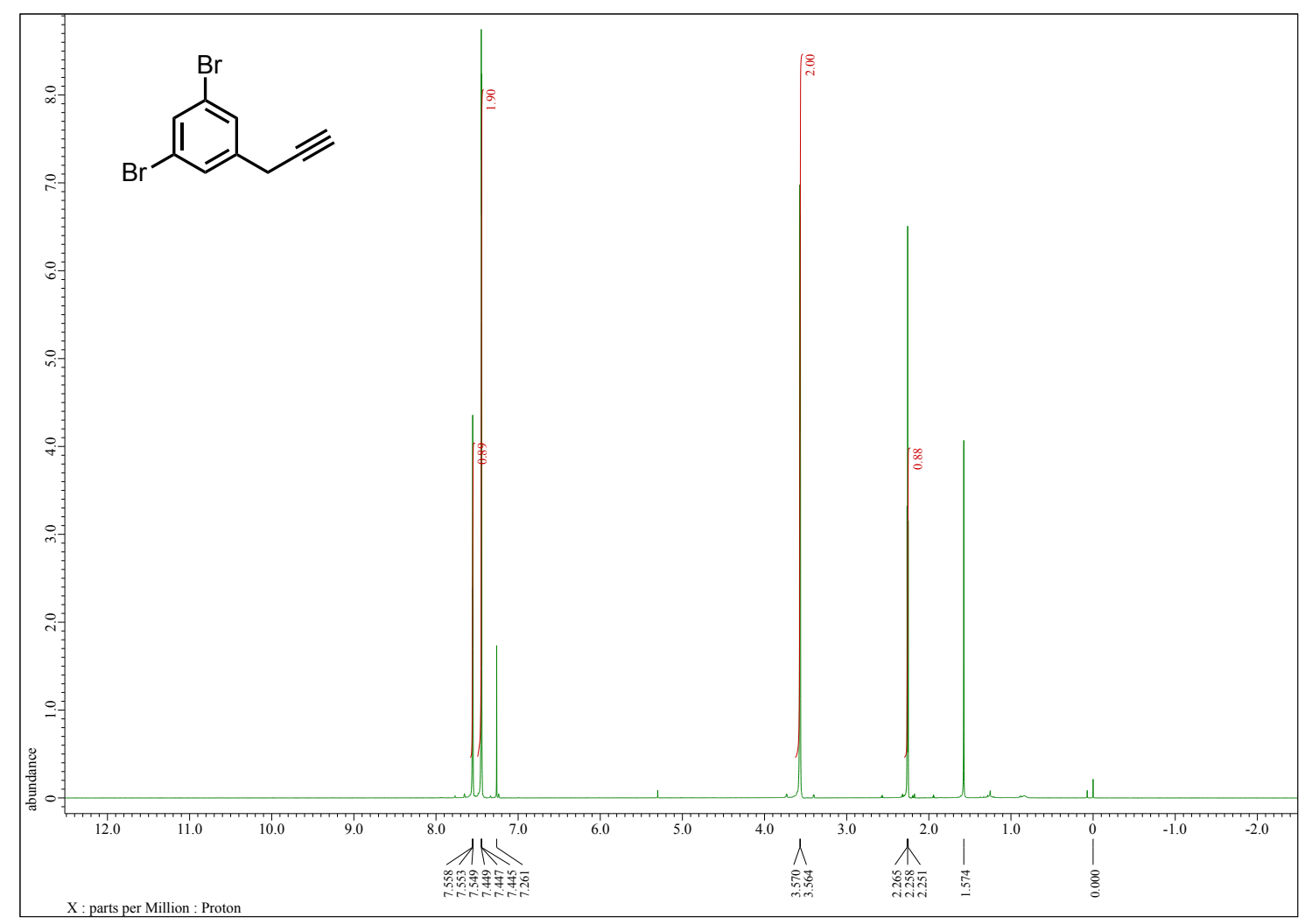

Figure S38. ${ }^{1} \mathrm{H}$ NMR of $4 \mathbf{n}\left(\mathrm{CDCl}_{3}\right)$.

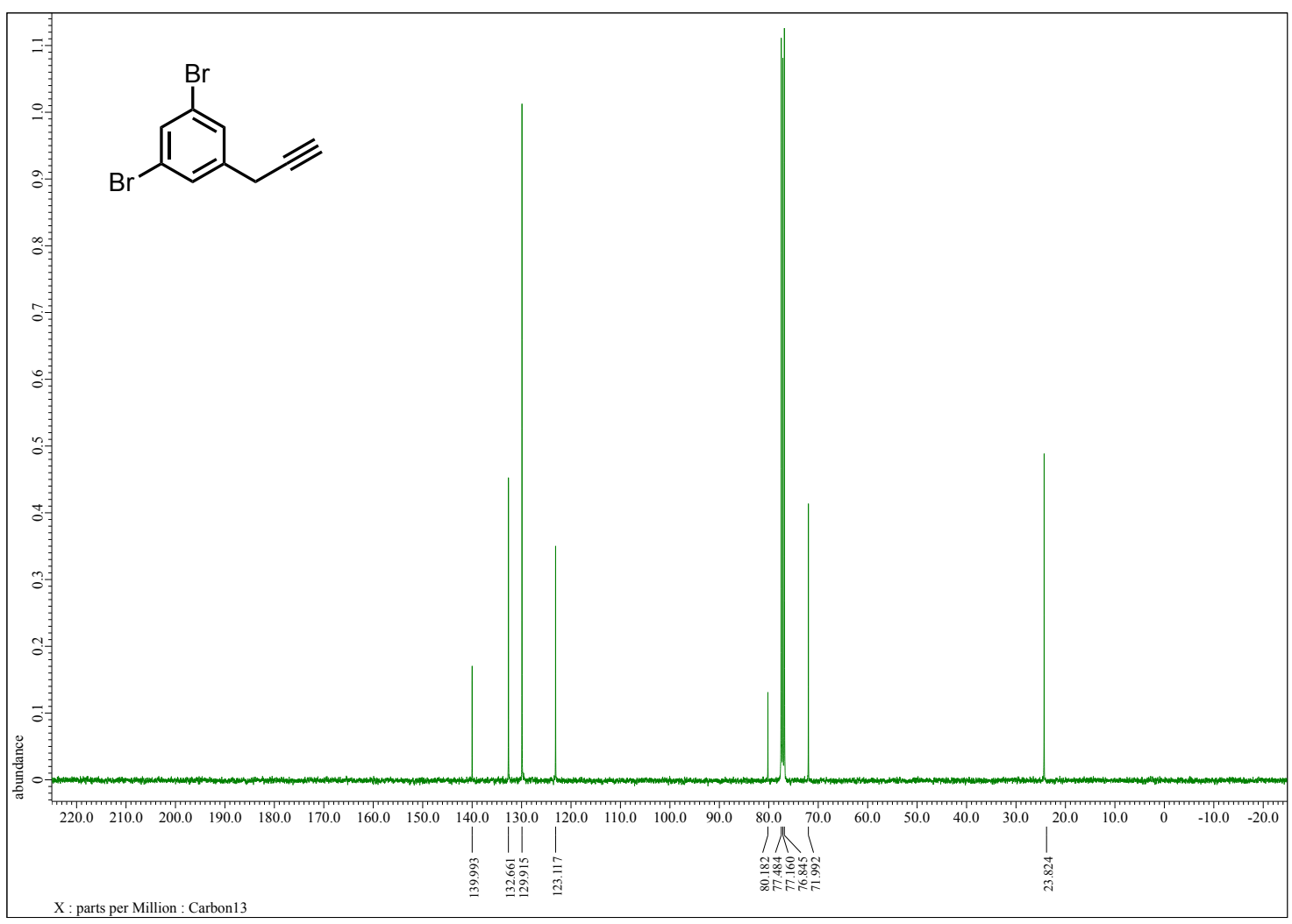

Figure S39. ${ }^{13} \mathrm{C}$ NMR of $4 \mathrm{n}\left(\mathrm{CDCl}_{3}\right)$. 


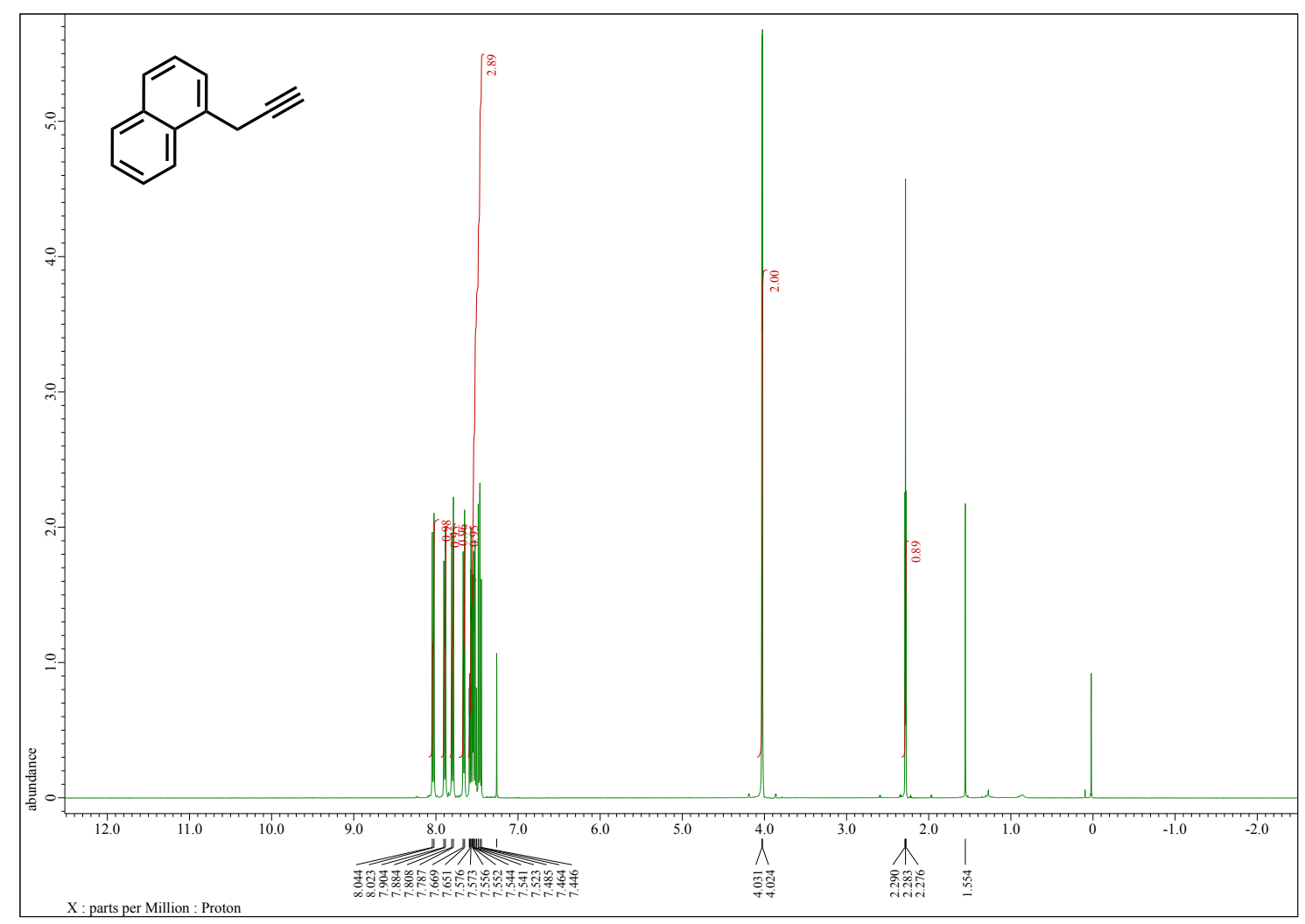

Figure S40. ${ }^{1} \mathrm{H}$ NMR of $40\left(\mathrm{CDCl}_{3}\right)$.

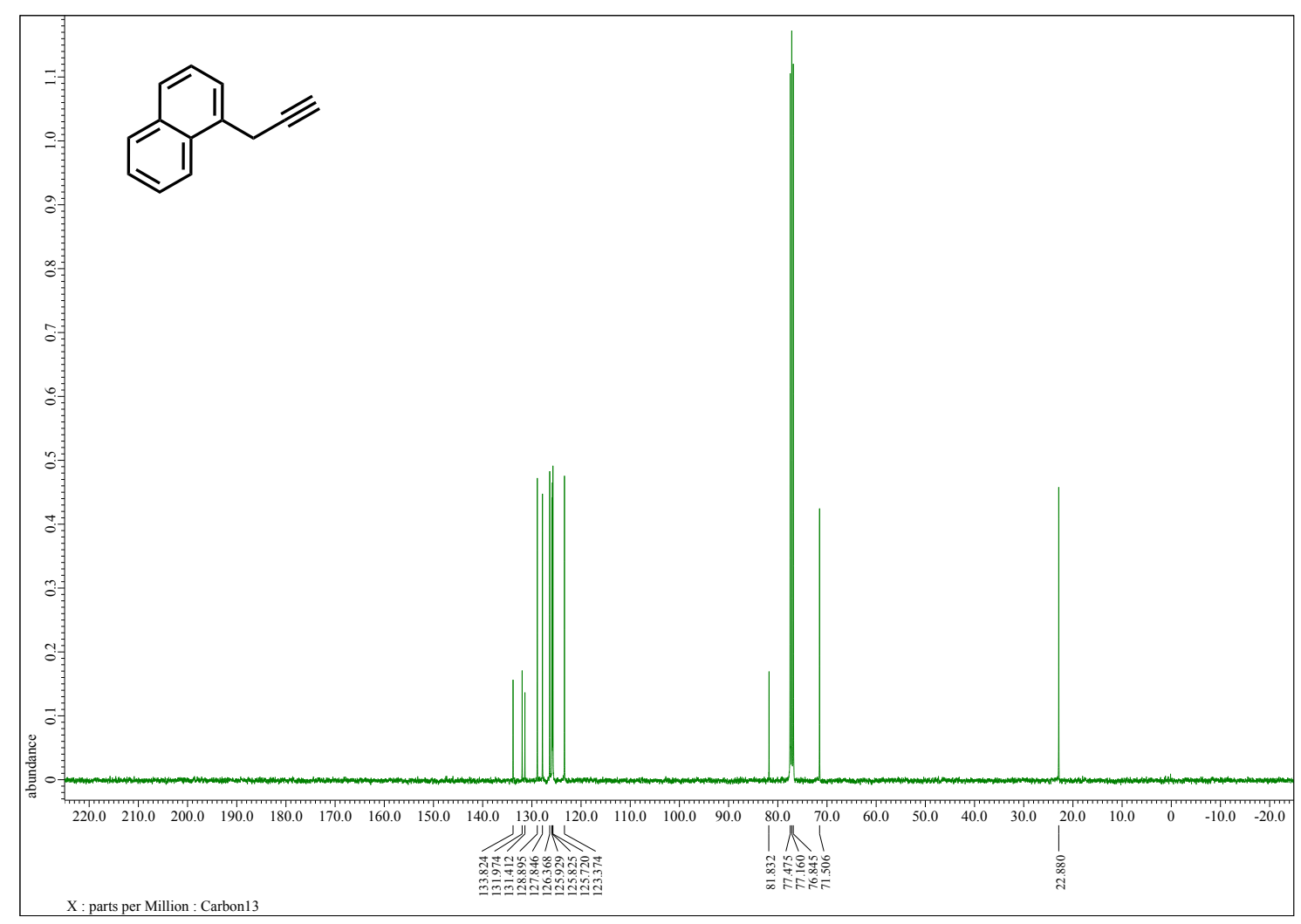

Figure S41. ${ }^{13} \mathrm{C}$ NMR of $40\left(\mathrm{CDCl}_{3}\right)$. 


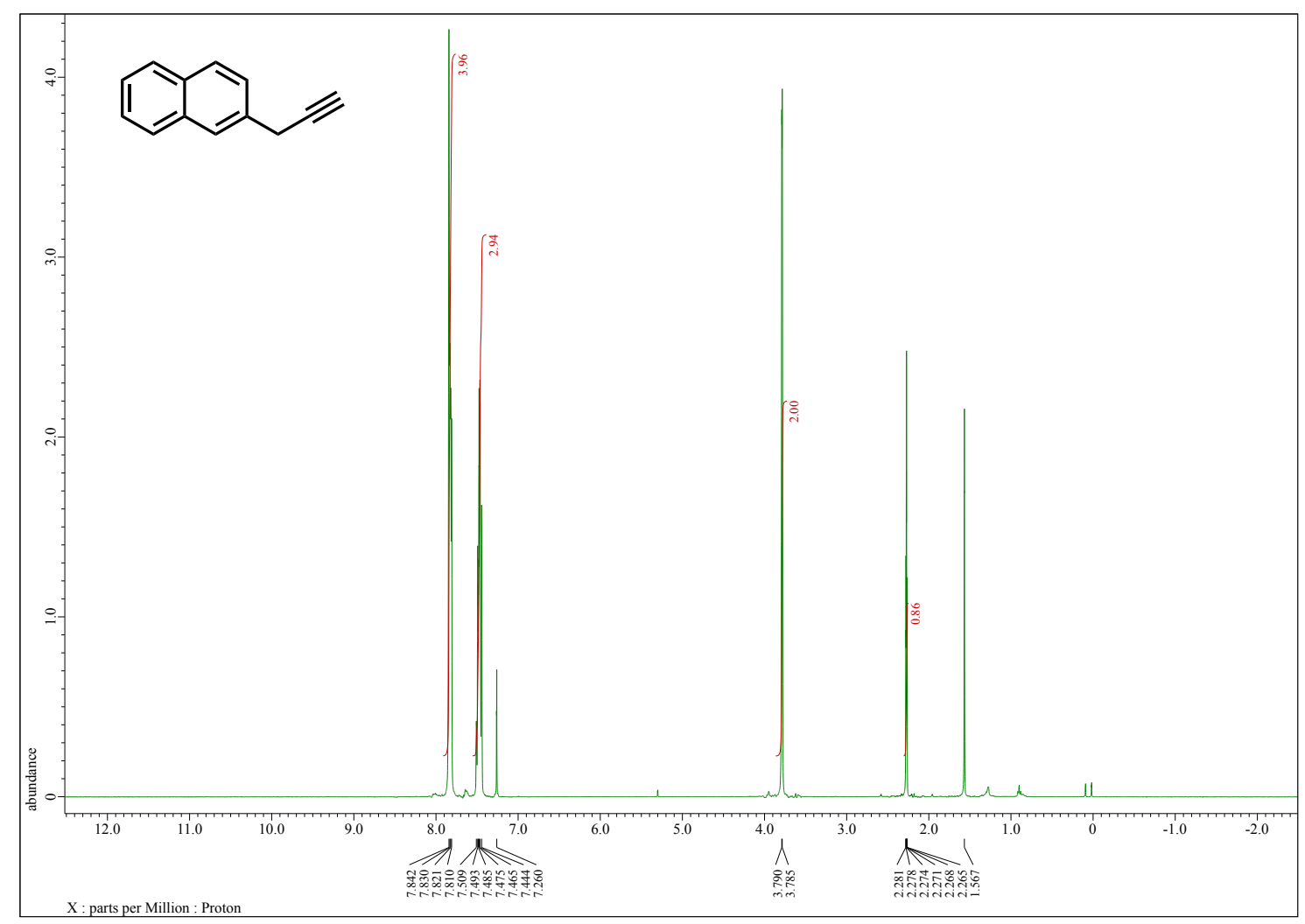

Figure $\mathbf{S 4 2 .}{ }^{1} \mathrm{H} \mathrm{NMR}$ of $\mathbf{4 p}\left(\mathrm{CDCl}_{3}\right)$.

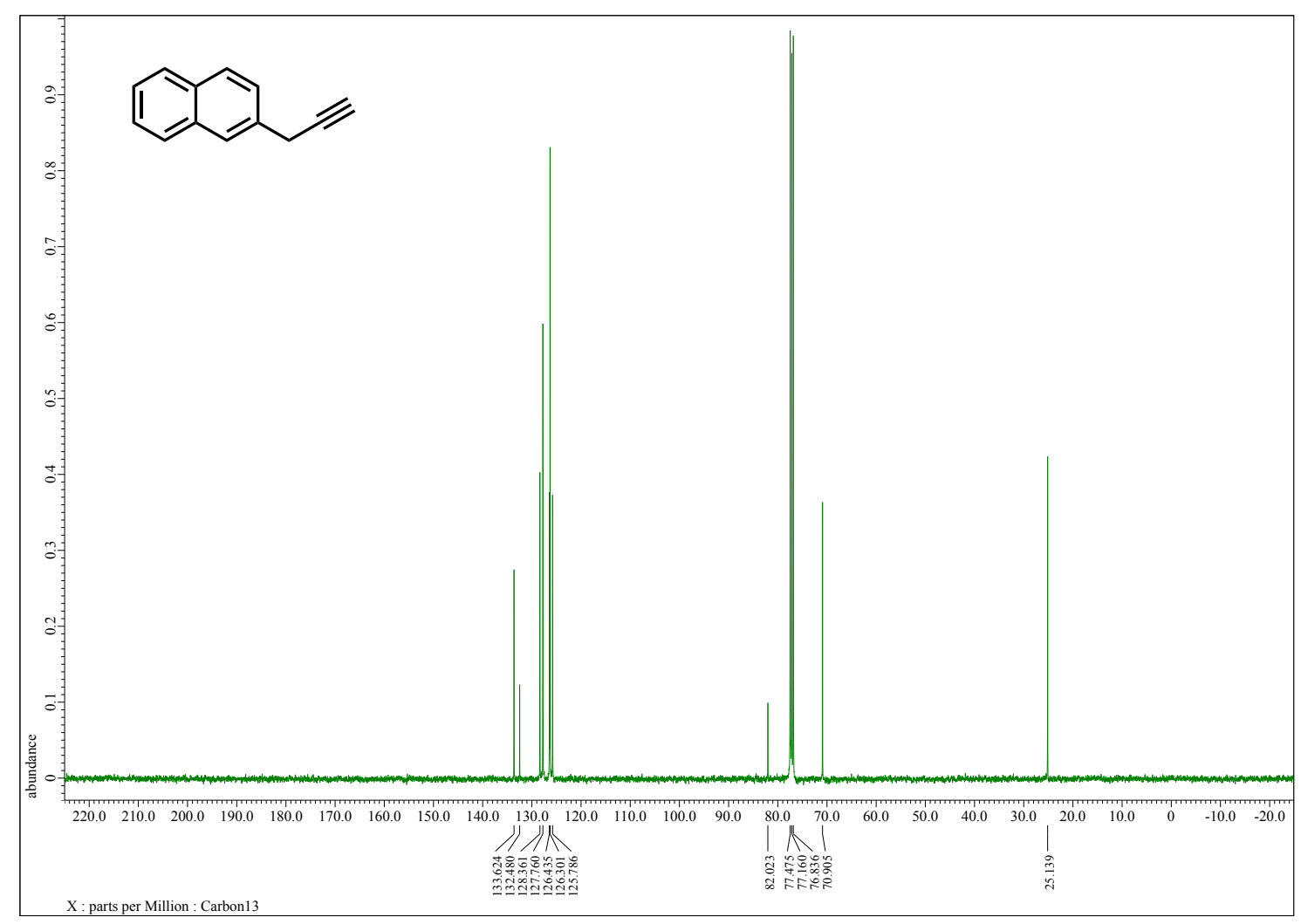

Figure S43. ${ }^{13} \mathrm{C}$ NMR of $4 p\left(\mathrm{CDCl}_{3}\right)$. 


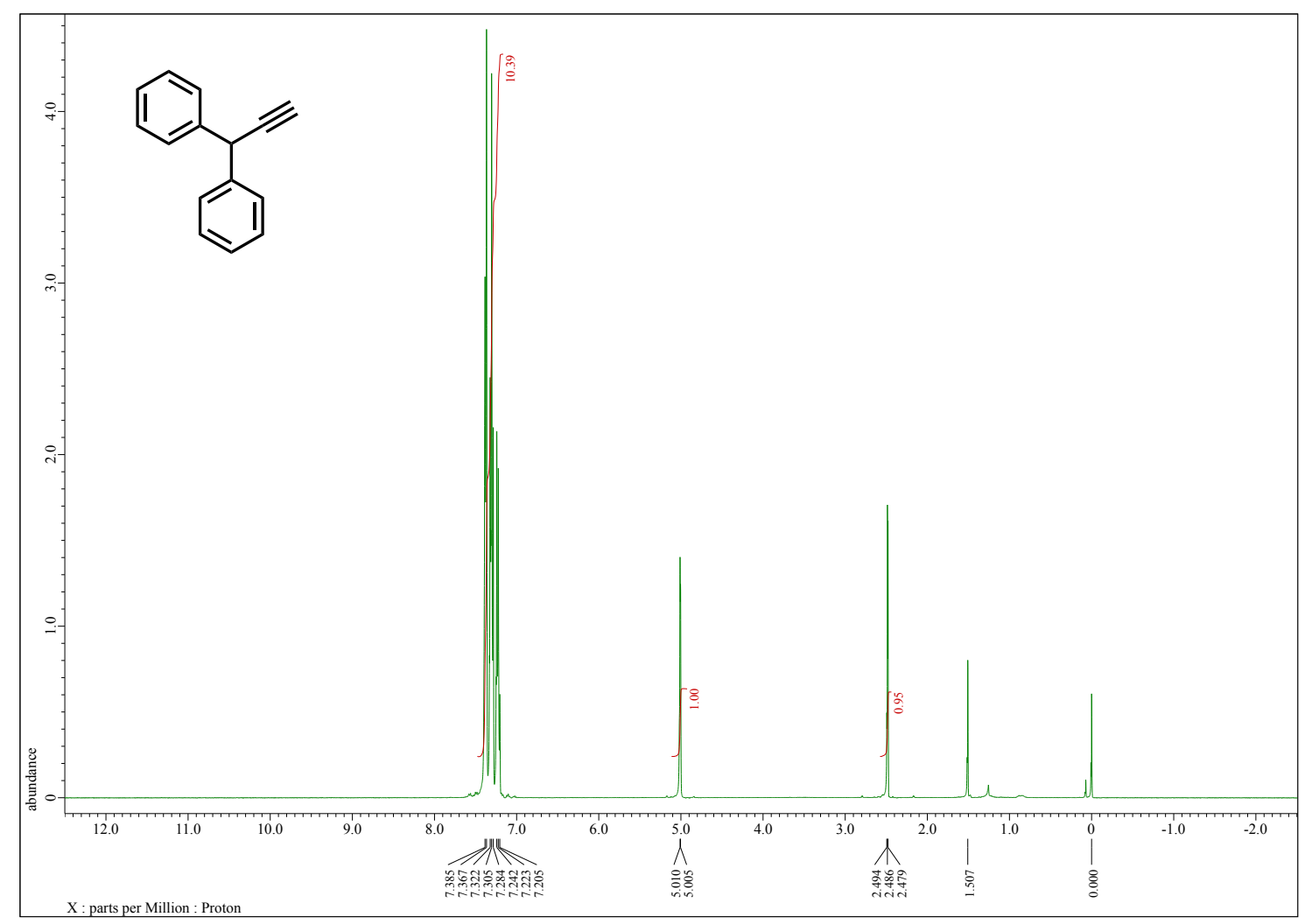

Figure $\mathbf{S 4 4} .{ }^{1} \mathrm{H} \mathrm{NMR}$ of $\mathbf{4 q}\left(\mathrm{CDCl}_{3}\right)$.

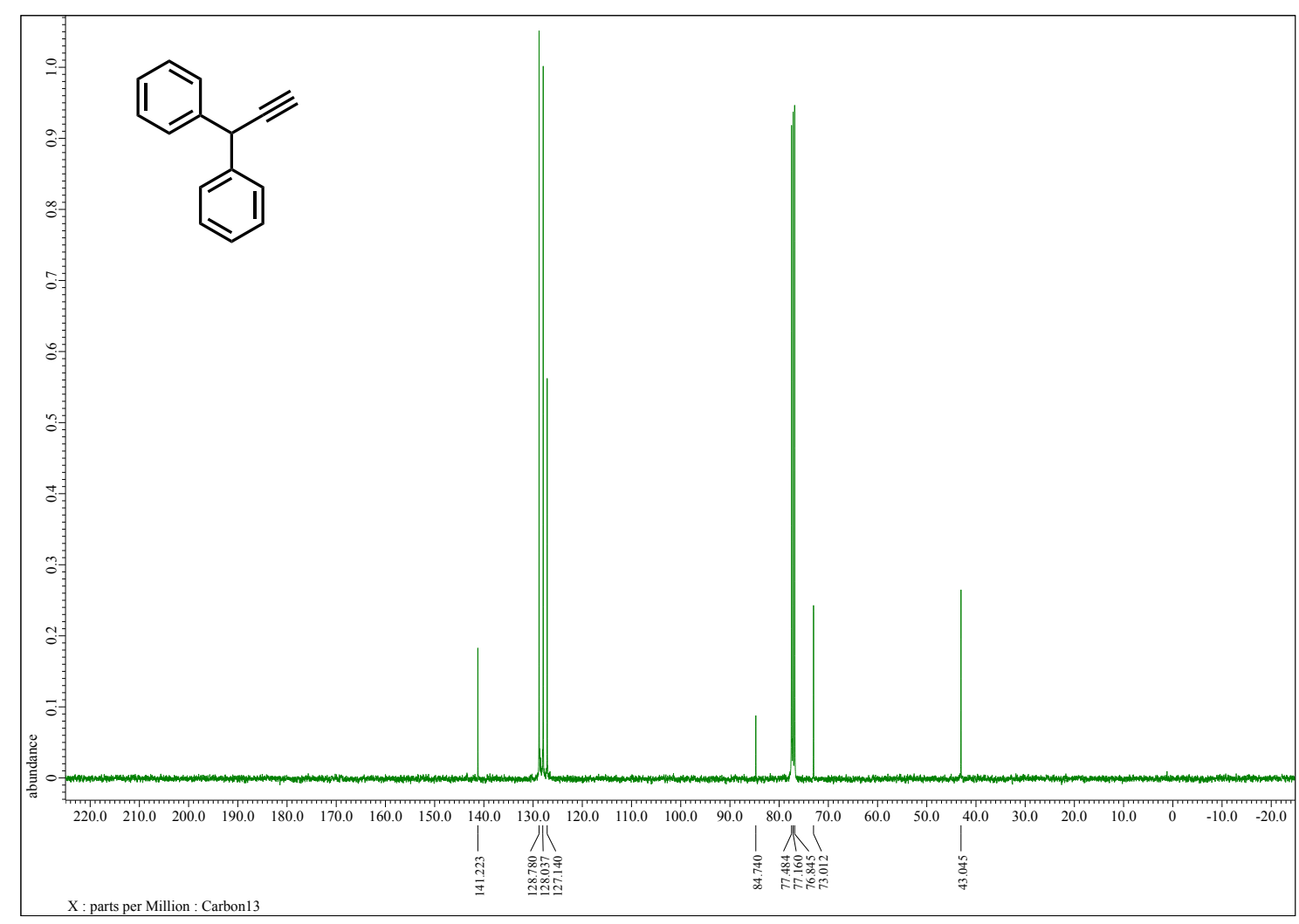

Figure S45. ${ }^{13} \mathrm{C}$ NMR of $4 q\left(\mathrm{CDCl}_{3}\right)$. 


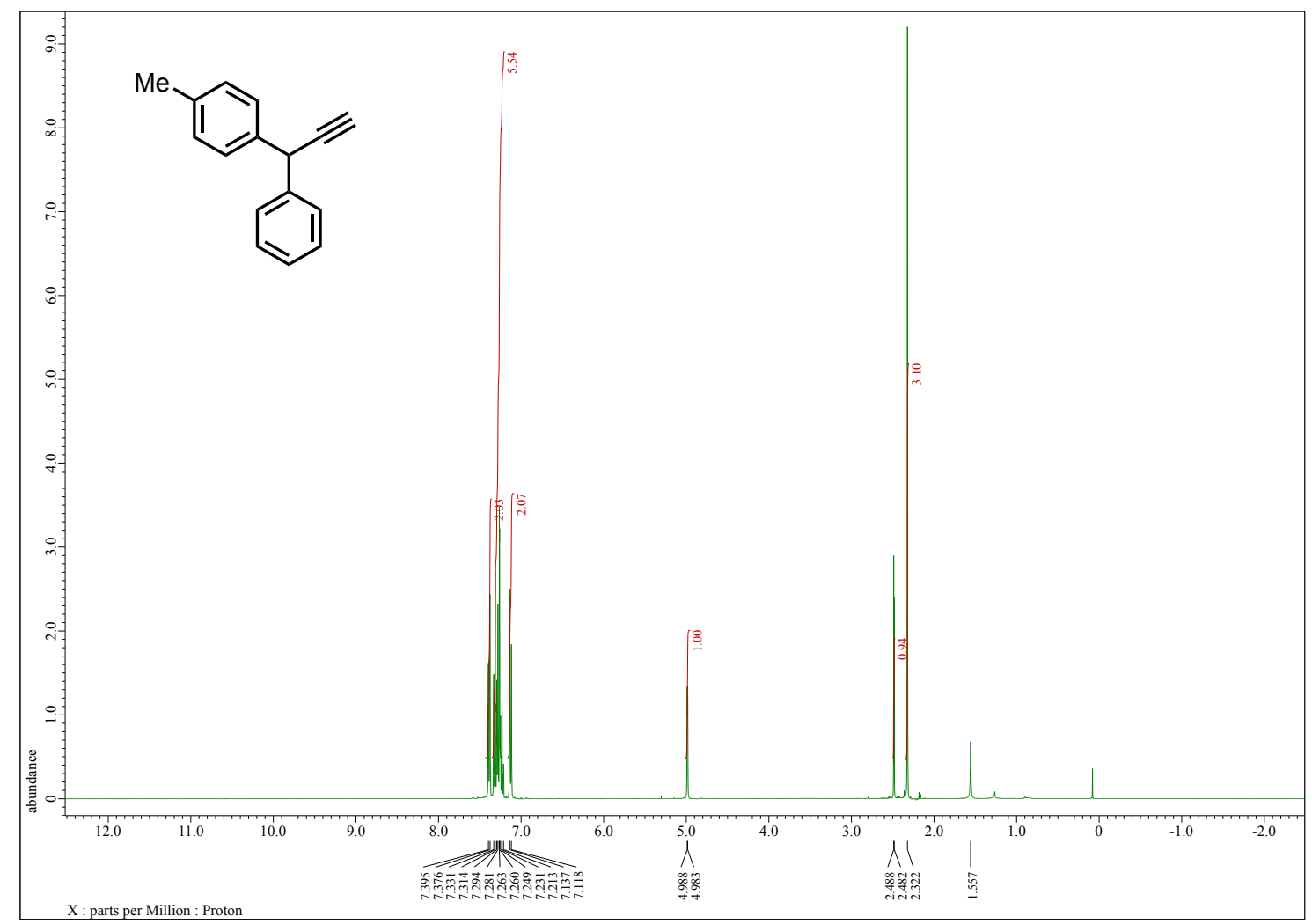

Figure S46. ${ }^{1} \mathrm{H}$ NMR of $4 r\left(\mathrm{CDCl}_{3}\right)$.

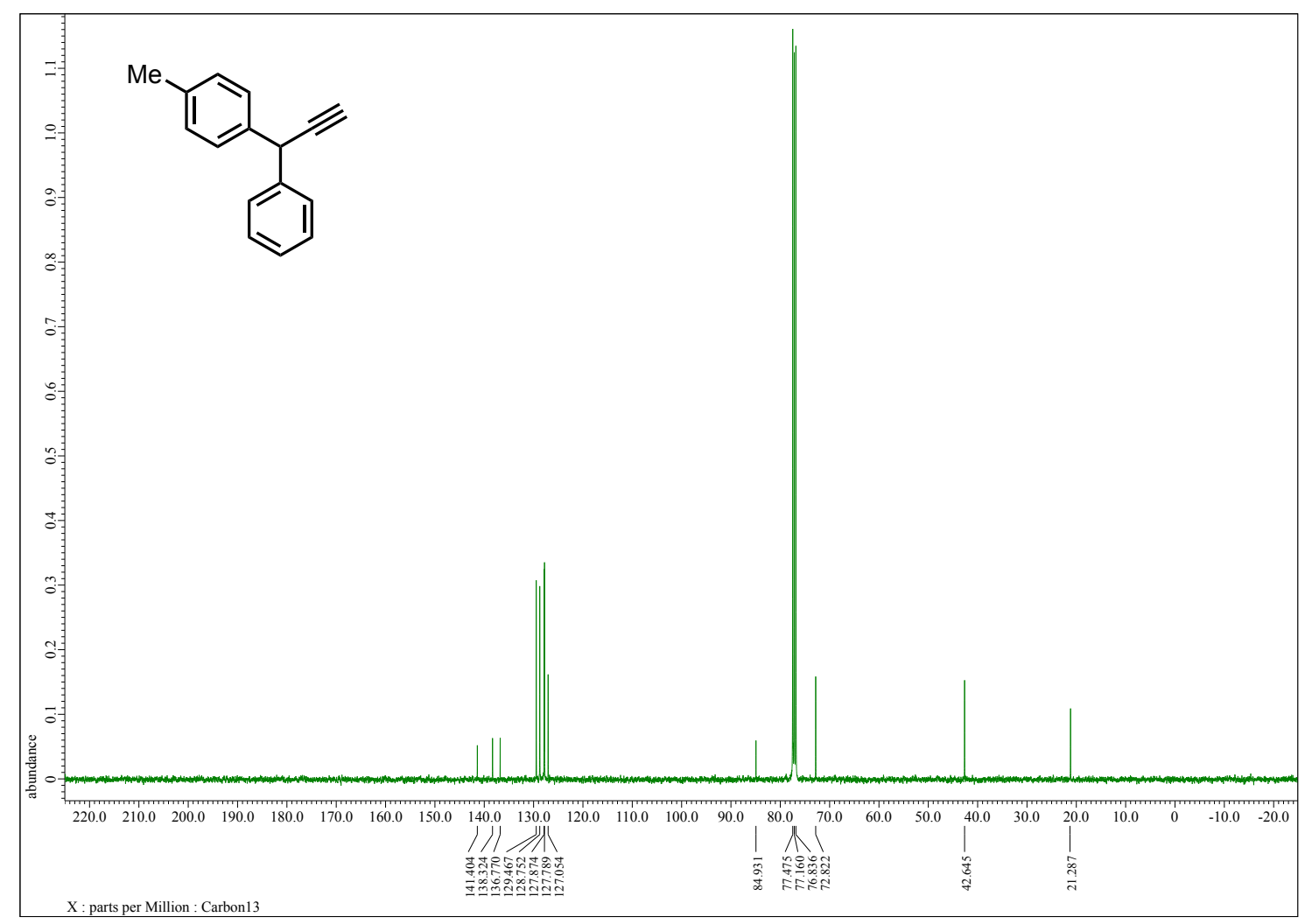

Figure $\mathbf{S 4 7 .}{ }^{13} \mathrm{C} \mathrm{NMR}$ of $4 \mathbf{r}\left(\mathrm{CDCl}_{3}\right)$. 


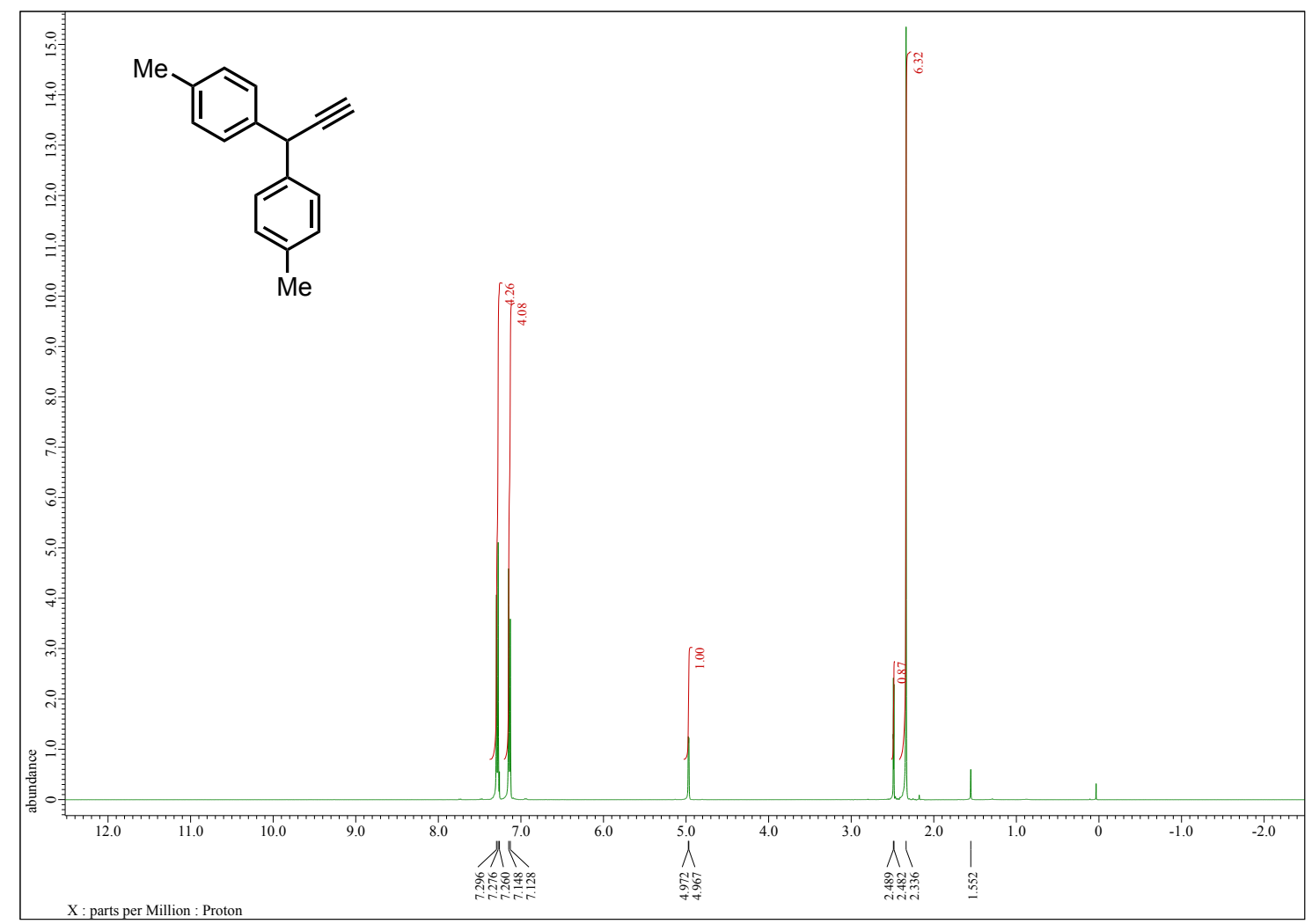

Figure S48. ${ }^{1} \mathrm{H}$ NMR of $4 \mathbf{s}\left(\mathrm{CDCl}_{3}\right)$.

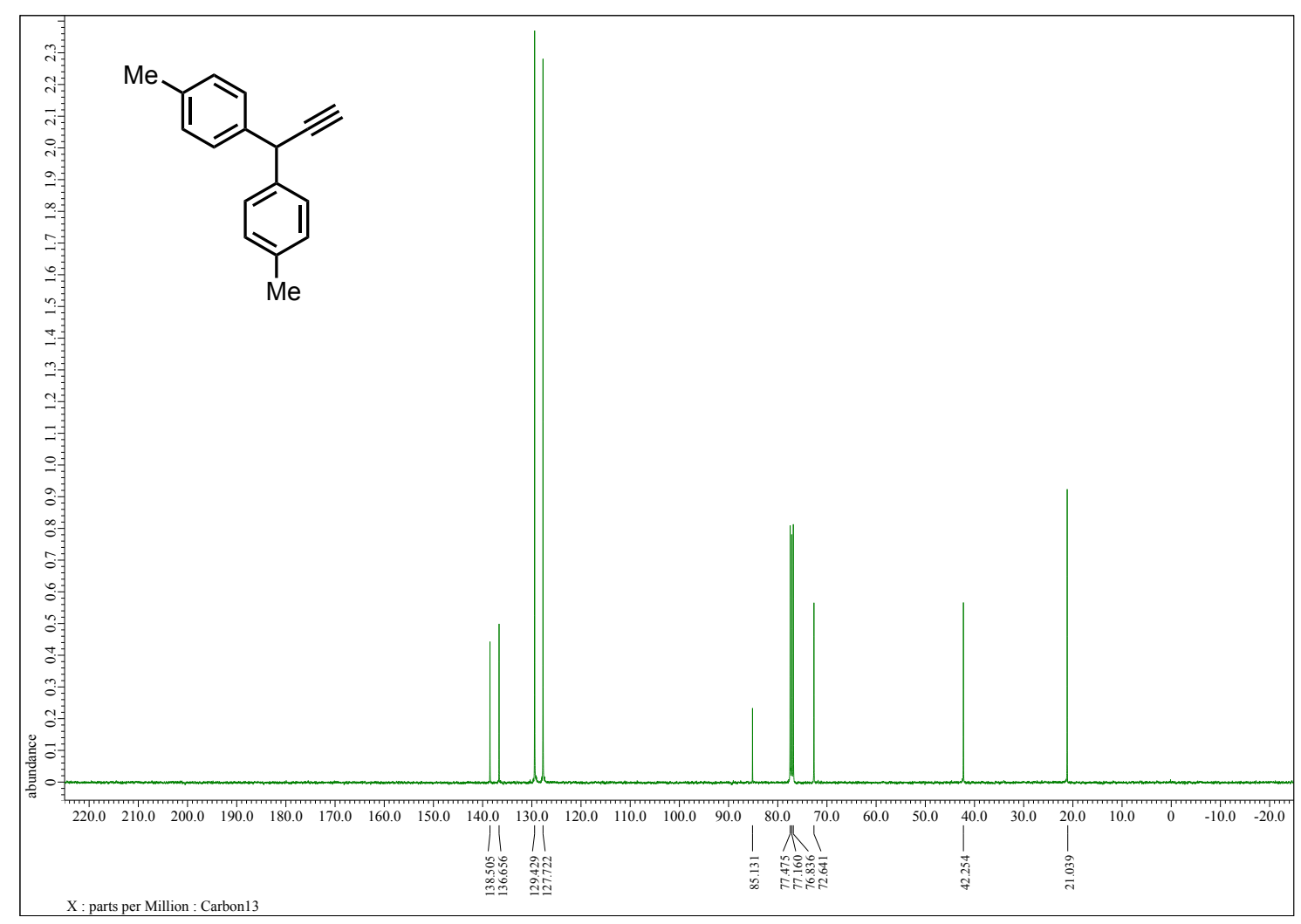

Figure S49. ${ }^{13} \mathrm{C} \mathrm{NMR}$ of $4 \mathrm{~s}\left(\mathrm{CDCl}_{3}\right)$. 


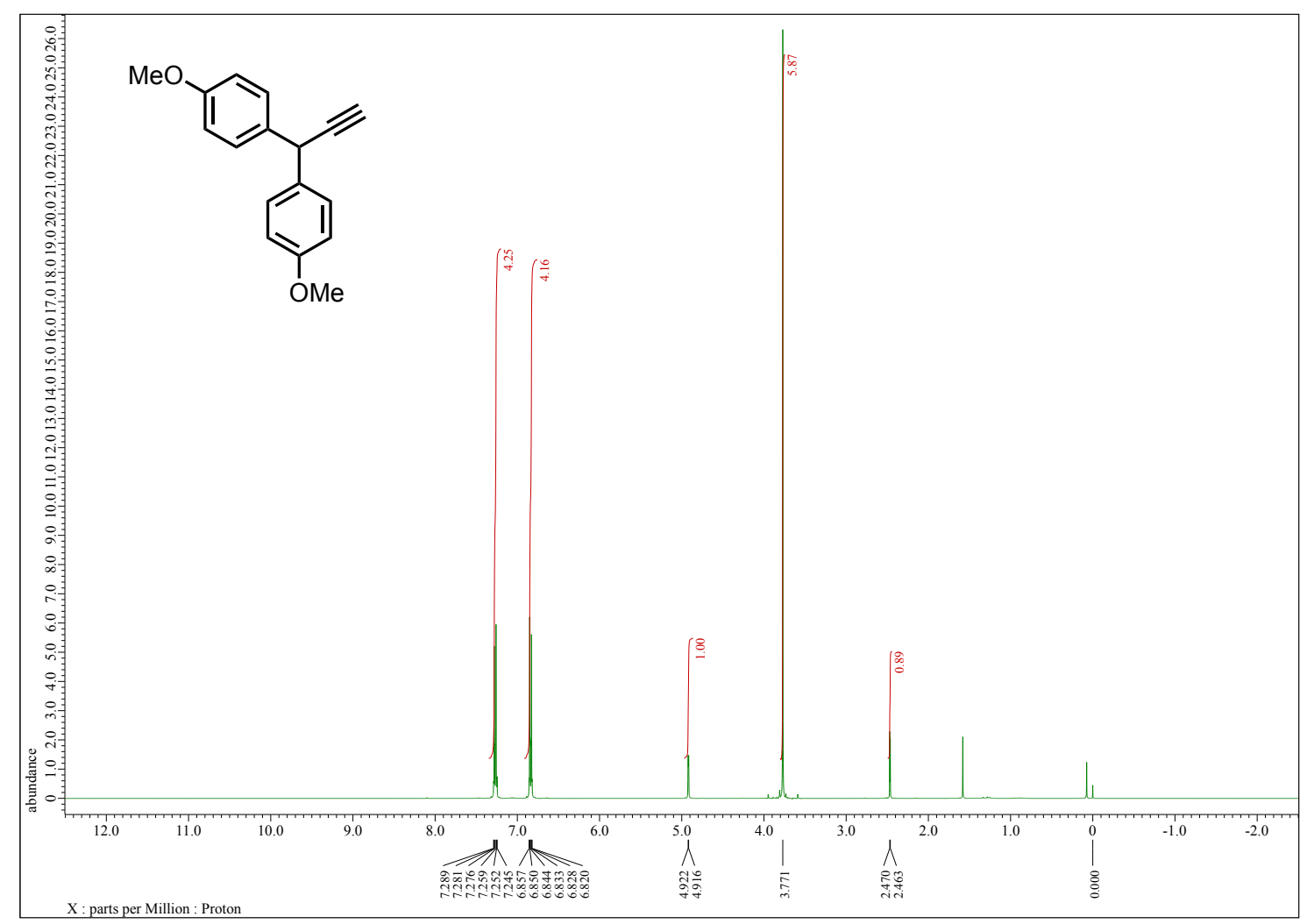

Figure S50. ${ }^{1} \mathrm{H}$ NMR of $\mathbf{4 t}\left(\mathrm{CDCl}_{3}\right)$.

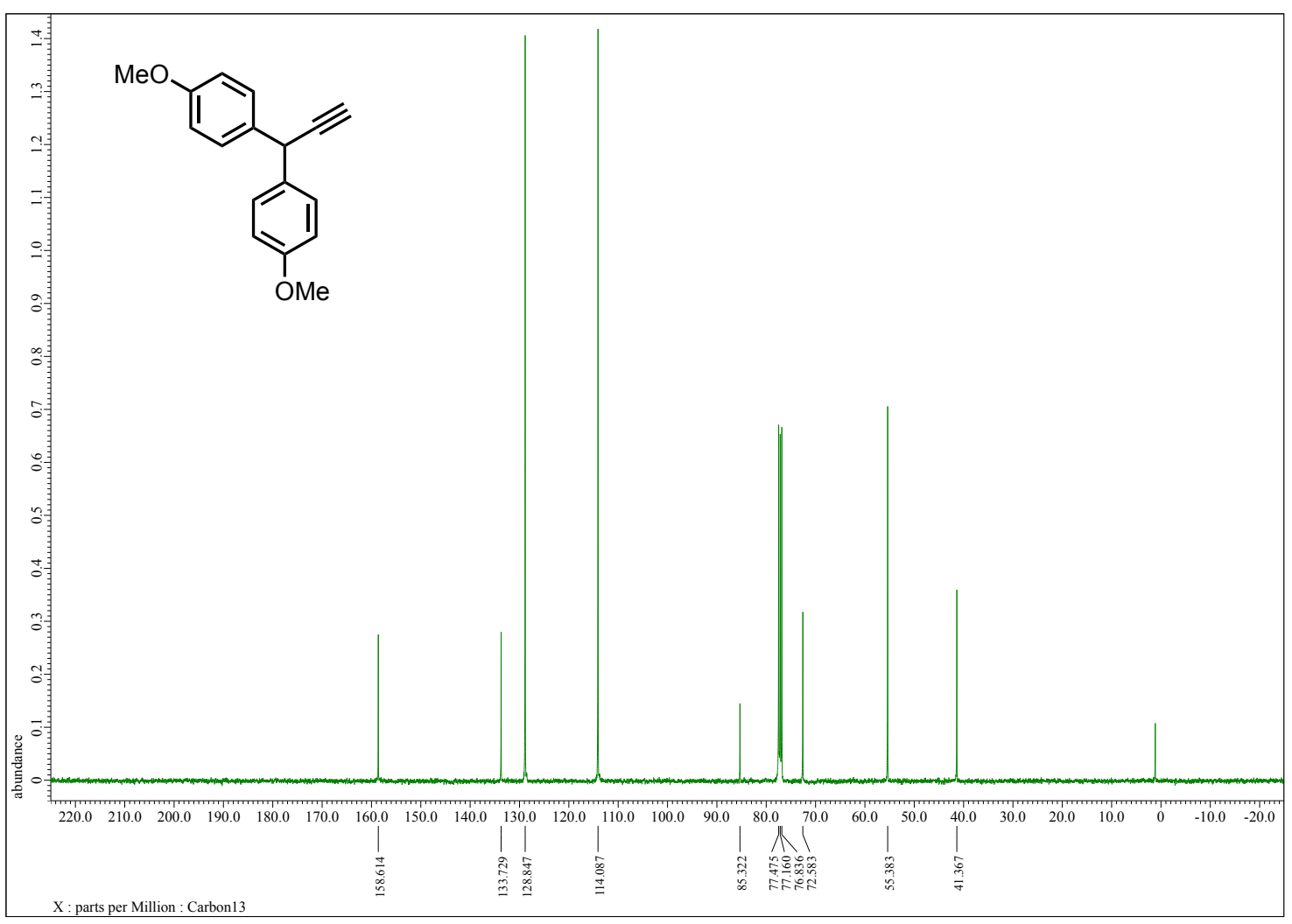

Figure S51. ${ }^{13} \mathrm{C}$ NMR of $4 t\left(\mathrm{CDCl}_{3}\right)$. 


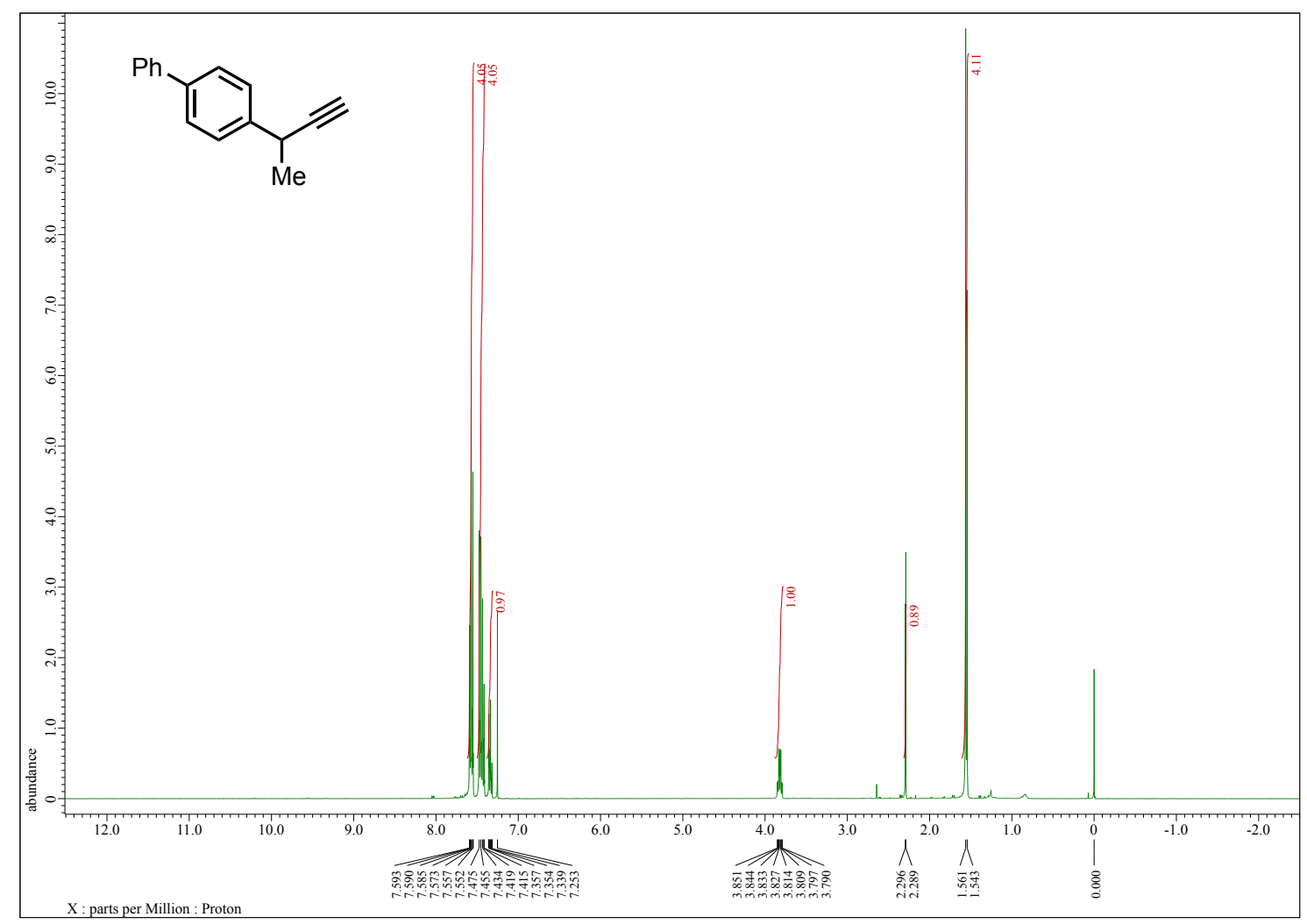

Figure S52. ${ }^{1} \mathrm{H}$ NMR of $\mathbf{4 u}\left(\mathrm{CDCl}_{3}\right)$.

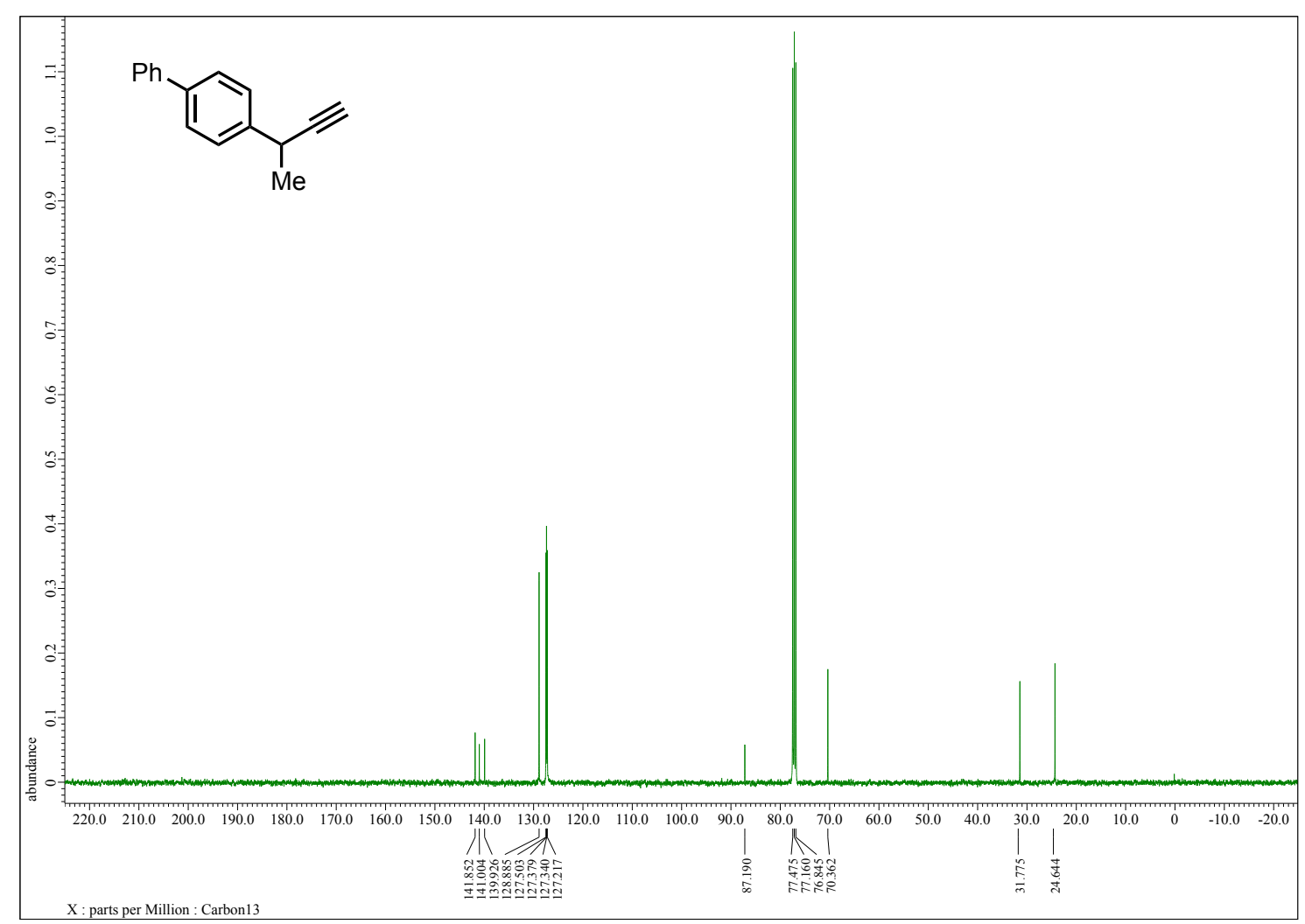

Figure S53. ${ }^{13} \mathrm{C}$ NMR of $4 \mathbf{u}\left(\mathrm{CDCl}_{3}\right)$. 


\section{References}

S1. Nishibayashi, Y.; Wakiji, I.; Hidai, M. Novel Propargylic Substitution Reactions Catalyzed by ThiolateBridged Diruthenium Complexes via Allenylidene Intermediates. J. Am. Chem. Soc. 2000, 122, 11019-11020. S2. Schneider, L. M.; Schmiedel, V. M.; Pecchioli, T.; Lentz, D.; Merten, C.; Christmann, M. Asymmetric Synthesis of Carbocyclic Propellanes. Org. Lett. 2017, 19, 2310-2313.

S3. Norcross, B. E.; Klinedinst, P. E.; Westheimer, F. H. The Reduction of Olefinic Double Bonds with Dihydropyridines. J. Am. Chem. Soc. 1962, 84, 797-802.

S4. Petrone, D. A.; Isomura, M.; Franzoni, I.; Rössler, S. L.; Carreira, E. M. Allenylic Carbonates in Enantioselective Iridium-Catalyzed Alkylations. J. Am. Chem. Soc. 2018, 140, 4697-4704.

S5. Kalhor-Monfared, S.; Beauvineau, C.; Scherman, D.; Girard, C. Synthesis and Cytotoxicity Evaluation of Aryl Triazolic Derivatives and their Hydroxymethine Homologues against B16 Melanoma Cell Line. Eur. J. Med. Chem. 2016, 122, 436-441.

S6. Opsitnick, E. A.; Jiang, X.; Hollenbeck, A. N.; Lee, D. Hydrogen-Bond-Assisted Helical Folding of Propeller-Shaped Molecules: Effects of Extended $\pi$-Conjugation on Chiral Selection, Conformational Stability, and Exciton Coupling. Eur. J. Org. Chem. 2012, 708-720.

S7. Chinta, B. S.; Baire, B. Stereoselective, Cascade Synthesis of trans-Enynones through CouplingIsomerization Reaction. J. Org. Chem. 2015, 80, 10208-10217.

S8. Senda, Y.; Nakajima, K.; Nishibayashi, Y. Cooperative Catalysis: Enantioselective Propargylic Alkylation of Propargylic Alcohols with Enecarbamates Using Ruthenium/Phosphoramide Hybrid Catalysts. Angew. Chem. Int. Ed. 2015, 54, 4060-4064.

S9. Ghazvini, H. J.; Armaghan, M.; Janiak, C.; Balalaie, S.; Müller, T. J. J. Coupling-IsomerizationCycloisomerization Reaction (CICIR) - An Unexpected and Efficient Domino Approach to Luminescent 2-(Hydroxymethylene)indenones. Euj. J. Org. Chem. 2019, 7058-7062.

S10. Altimari, J. M.; Hockey, S. C.; Boshoff, H. I.; Sajid, A.; Henderson, L. C. Novel 1,4-Substituted1,2,3-Triazoles as Antitubercular Agents. ChemMedChem 2015, 10, 787-791.

S11. Yoshida, A.; Ikeda, M.; Hattori, G.; Miyake, Y.; Nishibayashi, Y. Cooperative Catalytic Reactions Using Organocatalysts and Transition Metal Catalysts: Enantioselective Propargylic Alkylation of Propargylic Esters with Aldehydes. Org. Lett. 2011, 13, 592-595.

S12. Zhang, H.; Tanimoto, H.; Morimoto, T.; Nishiyama, Y.; Kakiuchi, K. Regioselective Rapid Synthesis of Fully Substituted 1,2,3-Triazoles Mediated by Propargyl Cations. Org. Lett. 2013, 15, $5222-5225$.

S13. Mothe, S. R.; Lauw, S. J. L.; Kothandaraman, P.; Chan, P. W. H. Brønsted Acid-Catalyzed Cycloisomerization of But-2-yne-1,4-diols with or without 1,3-Dicarbonyl Compounds to Tri- and Tetrasubstituted Furans. J. Org. Chem. 2012, 77, 6937-6947.

S14. Yang, L.; Zeng, Q. Metal- and Acid-Free Methyl Triflate Catalyzed Meyer-Schuster Rearrangement. Synthesis 2017, 49, 3149-3156.

S15. Li, Y.; Wei, H.; Wu, D.; Li, Z.; Wang, W.; Yin, G. Nickel-Catalyzed Chemodivergent 1,1Difunctionalization of Unactivated $\alpha$-Olefins with Alkynyl Electrophiles and B2pin2. ACS Catal. 2020, 10, 4888-4894.

S16. Shiina, I.; Umezaki, Y.; Kuroda, N.; Iizumi, T.; Nagai, S.; Katoh, T. MNBA-Mediated $\beta$-Lactone 
Formation: Mechanistic Studies and Application for the Asymmetric Total Synthesis of Tetrahydrolipstatin. J. Org. Chem. 2012, 77, 4885-4901.

S17. Alford, J. S.; Spangler, J. E.; Davies, H. M. L. Conversion of Cyclic Ketones to 2,3-Fused Pyrroles and Substituted Indoles. J. Am. Chem. Soc. 2013, 135, 11712-11715.

S18. Girard, et al. Leukotriene antagonists, U.S. Patent 4,761,425, August 2, 1988.

S19. Yuki, M.; Miyake, Y.; Nishibayashi, Y. Preparation of Thiolate-Bridged Dinuclear Ruthenium Complexes Bearing a Phosphine Ligand and Application to Propargylic Reduction of Propargylic Alcohols with 2-Propanol. Organometallics 2010, 29, 5994-6001.

S20. Jensen, K. L.; Standley, E. A.; Jamison, T. F. Highly Regioselective Nickel-Catalyzed CrossCoupling of N-Tosylaziridines and Alkylzinc Reagents. J. Am. Chem. Soc. 2014, 136, 11145-11152.

S21. Kinena, L.; Leitis, G.; Kanepe-Lapsa, I.; Bobrovs, R.; Jaudzems, K.; Ozola, V.; Suna, E.; Jirgensons, A. Azole-Based Non-Peptidomimetic Plasmepsin Inhibitors. Arch. Pharm. Chem. Life. Sci. 2018, 351, e1800151.

S22. Rashad, A. A.; Acharya, K.; Haftl, A.; Aneja, R.; Dick, A.; Holmes, A. P.; Chaiken, I. Chemical Optimization of Macrocyclic HIV-1 Inactivators for Improving Potency and Increasing the Structural Diversity at the Triazole Ring. Org. Biomol. Chem. 2017, 15, 7770-7782.

S23. Li, Q.; Gau, H. Synthesis of Allenes via Nickel-Catalyzed Cross-Coupling Reaction of Propargylic Bromides with Grignard Reagents. Synlett 2012, 23, 747-750.

S24. Curtin, B. H.; Manoni, F.; Park, J.; Sisto, L. J.; Lam, Y.-H.; Gravel, M.; Roulston, A.; Harran, P. G. Assembly of Complex Macrocycles by Incrementally Amalgamating Unprotected Peptides with a Designed Four-Armed Insert. J. Org. Chem. 2018, 83, 3090-3108.

S25. Shu, C.; Li, L.; Chen, C.-B.; Shen, H.-C.; Ye, L.-W. Gold-Catalyzed 6-Exo-Dig Cycloisomerization: A Versatile Approach to Functionalized Phenanthrenes. Chem. Asian. J. 2014, 9, 1525-1529.

S26. Masters, K.-S.; Wallesch, M.; Bräse, S. ortho-Bromo(propa-1,2-dien-1-yl)arenes: Substrates for Domino Reactions. J. Org. Chem. 2011, 76, 9060-9067.

S27. Porter, N. A.; Hogenkamp, D. J.; Khouri, F. F. Aliene/Haloolefin Electrocyclic Reactions: A New Route to Stable Triarylmethyl Radicals. J. Am. Chem. Soc., 1990 112, 2402-2407.

S28. Xu, C.-F.; Xu, M.; Yang, L.-Q.; Li, C.-Y. Synthesis of Allenes via Gold-Catalyzed Intermolecular Reaction of Propargylic Alcohols and Aromatic Compounds. J. Org. Chem. 2012, 77, 3010-3016.

S29. Tóth, K.; Höfner, G.; Wanner, K. T. Synthesis and Biological Evaluation of Novel N-Substituted Nipecotic Acid Derivatives with an Alkyne Spacer as GABA Uptake Inhibitors. Bioorg. Med. Chem. 2018, 26, 3668-3687.

S30. G-Dayanandan, N.; Paulsen, J. L.; Viswanathan, K.; Keshipeddy, S.; Lombardo, M. N.; Zhou, W.; Lamb, K. M.; Sochia, A. E.; Alverson, J. B.; Priestley, N. D.; Wright, D. L.; Anderson, A. C. PropargylLinked Antifolates are Dual Inhibitors of Candida albicans and Candida glabrata. J. Med. Chem. 2014, 57, 2643-2656.

S31. Frisch, M. J.; Trucks, G. W.; Schlegel, H. B.; Scuseria, G. E.; Robb, M. A.; Cheeseman, J. R.; Scalmani, G.; Barone, V.; Petersson, G. A.; Nakatsuji, H.; Li, X.; Caricato, M.; Marenich, A. V.; Bloino, J.; Janesko, B. G.; Gomperts, R.; Mennucci, B.; Hratchian, H. P.; Ortiz, J. V.; Izmaylov, A. F.; 
Sonnenberg, J. L.; Williams-Young, D.; Ding, F.; Lipparini, F.; Egidi, F.; Goings, J.; Peng, B.; Petrone, A.; Henderson, T.; Ranasinghe, D.; Zakrzewski, V. G.; Gao, J.; Rega, N.; Zheng, G.; Liang, W.; Hada, M.; Ehara, M.; Toyota, K.; Fukuda, R.; Hasegawa, J.; Ishida, M.; Nakajima, T.; Honda, Y.; Kitao, O.; Nakai, H.; Vreven, T.; Throssell, K.; Montgomery, J. A., Jr.; Peralta, J. E.; Ogliaro, F.; Bearpark, M. J.; Heyd, J. J.; Brothers, E. N.; Kudin, K. N.; Staroverov, V. N.; Keith, T. A.; Kobayashi, R.; Normand, J.; Raghavachari, K.; Rendell, A. P.; Burant, J. C.; Iyengar, S. S.; Tomasi, J.; Cossi, M.; Millam, J. M.; Klene, M.; Adamo, C.; Cammi, R.; Ochterski, J. W.; Martin, R. L.; Morokuma, K.; Farkas, O.; Foresman, J. B.; Fox, D. J. Gaussian 16, Revision A.03; Gaussian, Inc.: Wallingford CT, 2016.

S32. (a) Hohenberg, P.; Kohn, W. Inhomogeneous Electron Gas. Phys. Rev. B 1964, 136, 864. (b)

Kohn, W.; Sham, L, J. Self-Consistent Equations Including Exchange and Correlation Effects. Phys.

Rev. A 1965, 140, 1133.

S33. Chai, J.-D.; Head-Gordon, M. Long-Range Corrected Hybrid Density Functionals with Damped Atom-Atom Dispersion Correction. Phys. Chem. Chem. Phys. 2008, 10, 6615-6620.

S34. Hehre, W. J.; Radom, L.; Schleyer, P. v. R.; Pople, J. A. Ab Initio Molecular Orbital Theory; Wiley: New York, 1986.

S35. Dolg, M.; Wedig, U.; Preuss, H. Energy-Adjusted ab initio Pseudopotentials for the First Row Transition Elements. J. Chem. Phys. 1987, 86, 866-872.

S36. (a) Miertuš, S.; Scrocco, E.; Tomasi, Electrostatic Interaction of a Solute with a Continuum. A Direct Utilizaion of AB initio Molecular Potentials for the Prevision of Solvent Effects. J. Chem. Phys. 1981, 55, 117-129. (b) Scalmani, G.; Frisch, M. Continuous Surface Charge Polarizable Continuum Models of Solvation. I. General Formalism. J. Chem. Phys. 2010, 132, 114110.

S37. Grimme, S. Supramolecular Binding Thermodynamics by Dispersion-Corrected Density Functional Theory. Chem. Eur. J. 2012, 18, 9955-9964.

S38. Luchini, G.; Alegre-Requena J. V.; Guan, Y.; Funes-Ardoiz, I.; Paton, R. S. GoodVibes 3.0.1, 2019 (http://doi.org/10.5281/zenodo.59524). 